\title{
Numerical bifurcation of Hamiltonian relative periodic orbits
}

\author{
Claudia Wulff and Frank Schilder \\ Department of Mathematics \\ University of Surrey \\ Guildford GU2 7XH, United Kingdom \\ email: c.wulff@surrey.ac.uk, f.schilder@surrey.ac.uk
}

April 18, 2009

\begin{abstract}
Relative periodic orbits (RPOs) are ubiquitous in symmetric Hamiltonian systems and occur for example in celestial mechanics, molecular dynamics and the motion of rigid bodies. RPOs are solutions which are periodic orbits of the symmetry-reduced system. In this paper we analyze certain symmetry-breaking bifurcations of RPOs in Hamiltonian systems with compact symmetry group and show how they can be detected and computed numerically. These are turning points of RPOs, relative period-doubling and relative period-halving bifurcations along branches of RPOs. In a co-moving frame the latter correspond to symmetry-breaking/symmetry-increasing pitchfork bifurcations or to period doubling/period-halving bifurcations. We apply our methods to the family of rotating choreographies which bifurcate from the famous Figure Eight solution of the three body problem as angular momentum is varied. We find that the family of choreographies rotating around the $e^{2}$-axis bifurcates to the family of rotating choreographies that connects to the Lagrange relative equilibrium. Moreover, we compute several relative period-doubling bifurcations and a turning point of the family of planar rotating choreographies which bifurcates from the Figure Eight solution when the third component of the angular momentum vector is varied.
\end{abstract}

AMS subject classification. 37G15, 37J20, 37M20, 70H33

Keywords. Numerical bifurcation analysis, symmetric Hamiltonian systems, relative periodic orbits.

\section{Contents}

1 Introduction

2 Persistence and numerical continuation of transversal relative equilibria and RPOs

2.1 Continuation of transversal Hamiltonian relative equilibria . . . . . . . . . . . . 4

2.1.1 Persistence of transversal relative equilibria . . . . . . . . . . . . . . . 5

2.1.2 Numerical computation of transversal relative equilibria . . . . . . . . 8

2.1.3 Numerical path-following of relative equilibria . . . . . . . . . . . . . 10

2.2 Persistence of transversal RPOs . . . . . . . . . . . . . . . . . . . . . . . . . . . . . . . . .

2.3 Numerical continuation of transversal RPOs . . . . . . . . . . . . . . . . . . 15

2.4 Numerical path-following of RPOs . . . . . . . . . . . . . . . . 19 
2.5 Turning points of relative equilibria and RPOs . . . . . . . . . . . . . . . 20

2.5.1 Turning points of Hamiltonian relative equilibria . . . . . . . . . . . 20

2.5.2 Turning points of RPOs . . . . . . . . . . . . . . . . 21

2.5.3 Detection and computation of turning points of relative equilibria and RPOs 22

3 Hamiltonian relative period doubling bifurcations 22

3.1 A theorem on relative period doubling bifurcations of RPOs . . . . . . . . . . . . 22

3.2 Detection of relative period doubling bifurcations . . . . . . . . . . . . . . 25

3.3 Computation of relative period doubling points and branch switching . . . . . . . 26

3.4 Detection and computation of relative period halving bifurcations . . . . . . . . 27

4 Application to rotating choreographies $\quad \mathbf{2 7}$

4.1 N-body problems and their symmetries . . . . . . . . . . . . . . . . . 28

4.2 Rotating choreographies of the three body problem . . . . . . . . . . . . . . . 29

4.3 Flip up pitchfork bifurcation of the type II rotating eight . . . . . . . . . . . . . 29

4.4 Turning points and relative period doubling bifurcations of type III rotating chore-

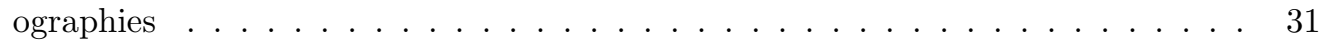

5 Conclusion

\section{Introduction}

Relative periodic orbits (RPOs) are ubiquitous in symmetric Hamiltonian systems. They are periodic orbits of the symmetry reduced system whereas relative equilibria are equilibria of the symmetry reduced system. In the original phase space relative equilibria drift along their group orbit, and RPOs represent a periodic vibrational dynamics superimposed with a drift along the symmetry group, e.g., a rotation. In recent years a lot of progress has been made in the bifurcation theory of Hamiltonian RPOs, see [13, 15, 20, 22]. However a general theory of generic bifurcations of RPOs so far only exists for dissipative systems [10, 21]. The additional structure of symmetric Hamiltonian systems changes the generic behaviour compared to nonHamiltonian symmetric systems dramatically. As a result of this, a general bifurcation theory of Hamiltonian RPOs and the parallel development of numerical methods for the detection and computation of those bifurcations are open problems. Recent progress in the continuation of normal periodic orbits of symmetric Hamiltonian systems has been made by Muñoz-Almaraz et al $[6,14]$. Chenciner et al [3] continue rotating choreographies of the three-body problems which bifurcate from the famous Figure Eight solution [4]. In [20] we developed a persistence theory for non-degenerate RPOs with generic drift-momentum pair (see Section 2 below for definitions of these terms). In [24] we have extended the numerical methods for the continuation of symmetric periodic orbits of general systems from [23] to Hamiltonian systems, based on the theoretical persistence results from [20].

In this paper we prove a persistence result for transversal Hamiltonian RPOs of compact group actions with generic drift-momentum pair, extending a result from [16] for relative equilibria. Transversal RPOs include turning points of RPOs in energy or momentum. Moreover, we prove a theorem on relative period doubling of RPOs with regular drift-momentum pair. We then present under-determined nonlinear systems of equations that are satisfied by Hamiltonian relative equilibria and RPOs respectively, have regular derivative at their solutions and are therefore amenable to standard numerical path-following methods. In this way we extend related results in [14] to Hamiltonian relative equilibria with non-abelian symmetry groups and to RPOs. 
Moreover, we develop algorithms for the computation of turning points of Hamiltonian relative equilibria and RPOs and for the computation of relative period doubling and relative period halving bifurcations of RPOs. We continue in a conserved quantity of the system, which is either the energy or a component of the momentum map. The list of bifurcations of Hamiltonian relative equilibria and RPOs which we study is by no means exhaustive. In particular, in this paper we assume that the spatial symmetry of the relative equilibrium or RPO, respectively, is trivial (by reducing the dynamics onto the fixed point space of the spatial symmetry of the solutions). Hence, we do not deal with bifurcations breaking the spatial symmetry. Although we present new theoretical results, the emphasis in this paper is the "transformation" of theoretical persistence and bifurcation results into efficient algorithms for numerical path-following and the numerical computation of bifurcations.

The paper is structured as follows: The topic of Section 2 is the continuation of transversal Hamiltonian relative equilibria and RPOs. First, in Section 2.1, we review the notion of transversal relative equilibria and their persistence and present a numerical method for their computation extending results from [14]. In Section 2.2 we introduce so-called transversal RPOs. These generalize the concept of transversal relative equilibria introduced in [16]. The non-degeneracy condition that we required in earlier works $[19,20,24]$ is a more restrictive condition. Then, in Sections 2.3 and 2.4, we generalize our earlier numerical continuation methods [24] for nondegenerate RPOs to transversal RPOs of compact group actions. In Section 2.5 we numerically analyze turning points of relative equilibria and RPOs which are continued in some component of the momentum or in energy (in the case of RPOs). These occur at transversal, but degenerate relative equilibria and RPOs. We present numerical methods for their detection and computation.

In Section 3 we present a theorem on relative period doubling bifurcations of non-degenerate Hamiltonian RPOs with generic drift-momentum pair. In a co-moving frame these correspond to period-doubling or symmetry breaking pitchfork bifurcations (as analyzed in [5] for nonHamiltonian systems with discrete symmetries). We then present numerical methods for the detection and computation of relative period doubling and relative period halving bifurcations of Hamiltonian RPOs extending related methods for dissipative systems from [24].

In Section 4 we apply our results to rotating choreographies of the three-body problem, which bifurcate from the famous Figure Eight solution of Chenciner and Montgomery [4]. It is well-known that one of the non-planar rotating Figure Eight families connects to the Lagrange relative equilibrium (for example, see the discussion in [3]). We find that the other non-planar family of rotating choreographies bifurcates in a relative period halving bifurcation to the family of rotating choreographies that connects to the Lagrange relative equilibrium. Moreover, we find several relative period-doubling bifurcations and a turning point of the planar family of rotating choreographies, which bifurcates from the Figure Eight solution when the third component of the angular momentum vector is varied.

\section{Persistence and numerical continuation of transversal relative equilibria and RPOs}

In this section we present methods for the continuation of transversal RPOs extending results of $[20,23]$. We start with the simpler case of continuation of transversal relative equilibria and extend results of [14] in Section 2.1. Then we prove a persistence result for transversal RPOs (Section 2.2). In Sections 2.3 and 2.4 we develop a method for the path-following of transversal RPOs, and in Section 2.5 we show how to detect and compute turning points of relative equilibria and RPOs. 


\subsection{Continuation of transversal Hamiltonian relative equilibria}

We consider a Hamiltonian system

$$
\dot{x}=f_{H}(x)=\mathbb{J} \nabla H(x)
$$

with Hamiltonian (energy) $H(x)$ on an open subset $\mathcal{X}$ of a finite-dimensional symplectic vector space $\mathbb{R}^{2 d}$ with symplectic structure matrix $\mathbb{J}$ (i.e., $\mathbb{J}$ is skew-symmetric and invertible). Let

$$
\Omega(v, w)=\left\langle\mathbb{J}^{-1} v, w\right\rangle
$$

be the symplectic form generated by $\mathbb{J}$. Let $\Phi^{t}\left(x_{0}\right)$ denote the flow of $(2.1)$, i.e., $x(t)=\Phi^{t}\left(x_{0}\right)$ is a solution of $(2.1)$ with initial value $x(0)=x_{0}$. Then the energy $H(x)$ is a conserved quantity of (2.1): $H\left(\Phi^{t}\left(x_{0}\right)\right)=H\left(x_{0}\right)$ for all $x_{0}, t$. We assume that a finite-dimensional compact Lie group $\Gamma$ acts on $\mathcal{X}$ symplectically (i.e., $\Omega$ is $\Gamma$-invariant) and that the Hamiltonian $H$ is $\Gamma$-invariant; this implies that $(2.1)$ is $\Gamma$-equivariant, i.e., $f_{H}(\gamma x)=\gamma f_{H}(x)$ for all $x \in \mathcal{X}, \gamma \in \Gamma$. So whenever $x(t)$ is a solution of (2.1) then so is $\gamma x(t)$, for $\gamma \in \Gamma$. We call the elements of $\Gamma$ the symmetries of (2.1). Let $\mathbf{g}=\mathcal{T}_{\text {id }} \Gamma$ denote the Lie algebra of $\Gamma$. Here $\mathcal{T}_{\text {id }} \Gamma$ denotes the tangent space of $\Gamma$ at the identity. By Noether's theorem, locally there is a conserved quantity $\mathbf{J}_{\xi}$ of (2.1) for each continuous symmetry $\xi \in \mathbf{g}$ of the system, and $\mathbf{J}_{\xi}$ is the Hamiltonian for the symplectic flow $x \rightarrow \exp (\xi t) x$, see, e.g., $[1,11]$. The map $\mathbf{J}_{\xi}(x)=\mathbf{J}(x)(\xi)$ is linear in $\xi$, so that $\mathbf{J}$ is a map from a neighbourhood of each $x \in \mathcal{X}$ to the dual $\mathbf{g}^{*}$ of $\mathbf{g}$, called the momentum map. Let $\mathrm{Ad}_{\gamma}$, $\gamma \in \Gamma$, denote the adjoint action of $\Gamma$ on $\mathbf{g}: \operatorname{Ad}_{\gamma} \eta=\gamma \eta \gamma^{-1}, \eta \in \mathbf{g}, \gamma \in \Gamma$, and $\operatorname{ad}_{\xi}, \xi \in \mathbf{g}$, be the infinitesimal adjoint action: $\operatorname{ad}_{\xi} \eta=\left.\frac{\mathrm{d}}{\mathrm{d} t} \operatorname{Ad}_{\exp (t \xi)} \eta\right|_{t=0}=[\xi, \eta]$. Then the co-adjoint action of $\Gamma$ on $\mathbf{g}^{*}$ is given by

$$
\gamma \mu=\left(\operatorname{Ad}_{\gamma}^{*}\right)^{-1} \mu, \quad \gamma \in \Gamma, \quad \mu \in \mathbf{g}^{*}
$$

where $\left(\operatorname{Ad}_{\gamma}^{*} \mu\right)(\eta):=\mu\left(\operatorname{Ad}_{\gamma} \eta\right), \eta \in \mathbf{g}, \gamma \in \Gamma$, and the infinitesimal coadjoint action is given by

$$
-\left(\operatorname{ad}_{\xi}^{*} \mu\right)(\eta)=\mu\left(\operatorname{ad}_{\xi} \eta\right), \quad \xi, \eta \in \mathbf{g}, \quad \mu \in \mathbf{g}^{*} .
$$

We assume throughout the paper that $\mathbf{J}$ is defined on the whole of $\mathcal{X}$ and is $\Gamma$-equivariant with respect to the $\Gamma$-action on $\mathcal{X}$ and the co-adjoint action on $\mathbf{g}^{*}$. Moreover, we choose an Ad-invariant inner product on $\mathbf{g}$ such that the adjoint action on $\mathbf{g}$ is by orthogonal matrices and the adjoint and co-adjoint actions can be identified.

As usual (c.f. [7]), for an action of a group $\Gamma$ on a space $\mathcal{X}$ we define the isotropy group or spatial symmetry group of $x \in \mathcal{X}$ as $\Gamma_{x}=\{\gamma \in \Gamma, \gamma x=x\}$. For any subgroup $K$ or element $\gamma \in \Gamma$ of $\Gamma$ we define the fixed point space of $K$ or $\gamma$ as $\operatorname{Fix} \mathcal{X}(K)=\{x \in \mathcal{X}, \gamma x=x \forall \gamma \in K\}$ and $\operatorname{Fix}_{\mathcal{X}}(\gamma)=\{x \in \mathcal{X}, \gamma x=x\}$, respectively. We denote by $N(K)=\left\{\gamma \in \Gamma, \gamma K \gamma^{-1}=K\right\}$ the normalizer of the subgroup $K$ of $\Gamma$. For any group $\Gamma$ define $\Gamma^{\text {id }}$ to be the component of $\Gamma$ containing the identity (identity component of $\Gamma$ ).

\section{Examples 2.1}

a) One of the most common symmetry groups that arise in applications is the group of rotational symmetries $\Gamma=\mathrm{SO}(3)$, see Section 4 below for an example from celestial mechanics. In this case the space of momenta is $\mathbf{g}^{*}=\operatorname{so}(3)^{*} \simeq \mathbb{R}^{3}$ and $\mathbf{J}: \mathcal{X} \rightarrow \mathbb{R}^{3}$ is the angular momentum. In this case $\mathbf{g}=\mathrm{so}(3) \simeq \mathbb{R}^{3}$ and the adjoint and co-adjoint actions are just the usual multiplication by matrices in $\mathrm{SO}(3)$. The identification $\operatorname{so}(3) \simeq \mathbb{R}^{3}$ is given by the map

$$
\xi=\left(\xi_{1}, \xi_{2}, \xi_{3}\right) \rightarrow\left(\begin{array}{ccc}
0 & \xi_{3} & -\xi_{2} \\
-\xi_{3} & 0 & \xi_{1} \\
\xi_{2} & -\xi_{1} & 0
\end{array}\right)
$$

Here $\xi$ is an infinitesimal rotation with frequency $\|\xi\|$ around the vector $\xi$. The Lie bracket becomes $[\xi, \eta]=\xi \times \eta$, where $\xi, \eta \in \mathbb{R}^{3} \simeq \operatorname{so}(3)$. 
b) The symmetry group $\mathrm{SO}(2)$ of rotations in the plane (see Section 4.4 for an example from celestial mechanics) is abelian, and so adjoint and coadjoint actions are trivial.

A point $\bar{x} \in \mathcal{X}$ lies on a relative equilibrium $\Gamma \bar{x}$ if there is some $\bar{\xi} \in \mathbf{g}$ such that $\bar{\xi} \bar{x}=f_{H}(\bar{x})$, i.e., the relative equilibrium through $\bar{x}$ is an equilibrium of the Hamiltonian system (2.1) in a frame moving with velocity $\bar{\xi}$ and an equilibrium of the symmetry-reduced system. We call $\bar{\xi}$ the drift velocity of the relative equilibrium at $\bar{x}$. Throughout the paper we assume, without loss of generality, that the isotropy $K=\Gamma_{\bar{x}}$ of the relative equilibrium is trivial, i.e., $K=\{\mathrm{id}\}$ (if not, we restrict the dynamics to the fixed point space $\operatorname{Fix}(K)$ of $K$ and replace $\Gamma$ by $N(K)$ so that $K$ is trivial).

Let $\bar{x}$ lie on a relative equilibrium with drift velocity $\bar{\xi}$ and momentum $\bar{\mu}=\mathbf{J}(\bar{x})$ at $\bar{x}$. There is a simple relation between the drift velocity and momentum of a relative equilibrium:

$$
\operatorname{ad}_{\bar{\xi}}^{*} \bar{\mu}=0
$$

which is implied by momentum conservation: $\bar{\mu}=\mathbf{J}(\bar{x})=\mathbf{J}\left(\Phi^{t}(\bar{x})\right)=\mathbf{J}(\exp (t \bar{\xi}) \bar{x})=\operatorname{Ad}_{\exp (-t \bar{\xi})}^{*}$, c.f. $[16,19]$. This relation is crucial for the problem of persistence to nearby momentum values, as we will see below.

\section{Examples 2.2}

a) In the case of the symmetry group $\mathrm{SO}(3)$, see Example 2.1, every relative equilibria is a rotating wave, i.e., stationary in a frame rotating with its drift velocity $\bar{\xi}$. In this case (2.3) means that (for $\xi \neq 0$ ) the rotation axis $\bar{\xi} /\|\bar{\xi}\|$ of a rotating wave is parallel to its angular momentum vector $\bar{\mu}$ : we have $\operatorname{ad}_{\bar{\xi}}^{*} \bar{\mu}=\bar{\xi} \times \bar{\mu}=0$.

b) The symmetry group $\mathrm{SO}(2)$ is abelian, and so for this symmetry group (2.3) is always true.

\subsubsection{Persistence of transversal relative equilibria}

Before we come to the numerical continuation of transversal RPOs we first present a persistence result for transversal relative equilibria with regular velocity-momentum pair and show how to continue them numerically.

Definition 2.3 [16, 19]

(i) We call pairs $(\xi, \mu) \in \mathbf{g} \oplus \mathbf{g}^{*}$ satisfying (2.3) velocity-momentum pairs and denote the space of velocity-momentum pairs by

$$
\left(\mathbf{g} \oplus \mathbf{g}^{*}\right)^{c}:=\left\{(\xi, \mu) \in \mathbf{g} \oplus \mathbf{g}^{*}, \quad \operatorname{ad}_{\xi}^{*} \mu=0\right\} .
$$

(ii) We define an action of $\Gamma$ on the space of velocity-momentum pairs as follows:

$$
\gamma(\xi, \mu)=\left(\operatorname{Ad}_{\gamma} \xi,\left(\operatorname{Ad}_{\gamma}^{*}\right)^{-1} \mu\right), \quad \text { for } \gamma \in \Gamma,(\xi, \mu) \in\left(\mathbf{g} \oplus \mathbf{g}^{*}\right)^{c} .
$$

For later purposes we define the isotropy subgroup $\Gamma_{(\xi, \mu)}$ of $(\xi, \mu) \in\left(\mathbf{g} \oplus \mathbf{g}^{*}\right)^{c}$ with respect to this action as

$$
\Gamma_{(\xi, \mu)}=\{\gamma \in \Gamma, \quad \gamma(\xi, \mu)=(\xi, \mu)\},
$$

denote its Lie algebra by $\mathbf{g}_{(\xi, \mu)}$ and let $r_{(\xi, \mu)}=\operatorname{dim} \mathbf{g}_{(\xi, \mu)}$. Moreover, we define the isotropy subgroup of $\xi \in \mathbf{g}$ as $\Gamma_{\xi}=\Gamma_{(\xi, 0)}=\left\{\gamma \in \Gamma, \operatorname{Ad}_{\gamma} \xi=\xi\right\}$ and the momentum isotropy subgroup of $\mu \in \mathbf{g}^{*}$ by $\Gamma_{\mu}=\Gamma_{(0, \mu)}=\left\{\gamma \in \Gamma,\left(\operatorname{Ad}_{\gamma}^{*}\right)^{-1} \mu=\mu\right\}$, denote their Lie algebras by $\mathbf{g}_{\xi}$ and $\mathbf{g}_{\mu}$, respectively, and define $r_{\xi}=\operatorname{dim} \mathbf{g}_{\xi}, r_{\mu}=\operatorname{dim} \mathbf{g}_{\mu}$. 
(iii) We call a velocity-momentum pair $(\xi, \mu) \in\left(\mathbf{g} \oplus \mathbf{g}^{*}\right)^{c}$ regular if $r_{(\xi, \mu)}$ is locally constant in the space of velocity-momentum pairs (2.4). We call $\xi \in \mathbf{g}$ regular if $r_{\xi}$ is locally constant in $\mathbf{g}$. We call $\mu \in \mathbf{g}^{*}$ regular if $r_{\mu}$ is locally constant in $\mathbf{g}^{*}$.

Remark 2.4 As shown in [16, 19], $(\xi, \mu) \in\left(\mathbf{g} \oplus \mathbf{g}^{*}\right)^{c}$ is regular if and only if $\mathbf{g}_{(\xi, \mu)}$ is the Lie algebra of a maximal torus (for a definition of a maximal torus see, e.g., [2]). In particular, for a regular velocity-momentum pair $(\xi, \mu)$ the isotropy sub-algebra $\mathbf{g}_{(\xi, \mu)}$ is abelian. Regular velocity-momentum pairs $(\xi, \mu)$ are generic in the space of velocity-momentum pairs $\left(\mathbf{g} \oplus \mathbf{g}^{*}\right)^{c}$. Regular $\mu \in \mathbf{g}^{*}$ are generic in $\mathbf{g}^{*}$ and regular $\xi \in \mathbf{g}$ are generic in $\mathbf{g}$. The velocity $\xi$ of a regular velocity-momentum pair $(\xi, \mu)$ is generically regular. In this case $\mathbf{g}_{(\xi, \mu)}=\mathbf{g}_{\xi}$ holds. Similarly, the momentum $\mu$ of a regular velocity-momentum pair $(\xi, \mu)$ is generically regular. In this case $\mathbf{g}_{(\xi, \mu)}=\mathbf{g}_{\mu}$ holds. These statements are needed later on.

\section{Examples 2.5}

a) In the case of the symmetry group $\mathrm{SO}(3)$, see Example 2.1, a velocity momentum pair $(\xi, \mu) \in \operatorname{so}(3) \oplus \operatorname{so}(3)^{*} \simeq \mathbb{R}^{3} \oplus \mathbb{R}^{3}$ satisfies $\xi \| \mu$ as we saw in Example 2.2. The action of $\Gamma=\mathrm{SO}(3)$ on the pair $(\xi, \mu)$ is the standard action of $\mathrm{SO}(3)$ on two copies of $\mathbb{R}^{3}$. Hence, $(\xi, \mu)$ is regular if and only if $(\xi, \mu) \neq 0$.

b) In the case of the symmetry group $\Gamma=\mathrm{SO}(2)$ the action of $\Gamma$ on velocity-momentum pairs $(\xi, \mu) \in \operatorname{so}(2) \oplus \operatorname{so}(2)^{*} \simeq \mathbb{R}^{2}$ is trivial and every velocity-momentum pair is regular.

Next, we describe the form that (2.1) takes in symmetry-adapted coordinates. This is needed for the derivation of the persistence result and the numerical continuation method for relative equilibria. Denote by $\mathcal{N}$ a normal space transverse to $\Gamma \bar{x}$ at $\bar{x}$, i.e., $\mathcal{T}_{\bar{x}} \mathcal{X}=\mathcal{T}_{\bar{x}} \Gamma \bar{x} \oplus \mathcal{N}$. Then $\mathcal{N}$ is a model for the space of group orbits $\mathcal{X} / \Gamma$ near $\bar{x}$. Moreover there are a choice of normal space $\mathcal{N}$ and coordinates $x \simeq(\gamma, v), \gamma \in \Gamma, v \in \mathcal{N}$, near $\Gamma \bar{x}$ such that $\bar{x} \simeq(\mathrm{id}, 0)$, and such that the dynamics in these coordinates takes the form [17]:

$$
\begin{aligned}
\dot{\gamma} & =\gamma f_{\Gamma}(\nu, w)=\gamma \mathrm{D}_{\nu} h(\nu, w), \\
\dot{\nu} & =f_{\mathcal{N}_{0}}(\nu, w)=\operatorname{ad}_{\mathrm{D}_{\nu} h(\nu, w)}^{*} \nu, \\
\dot{w} & =f_{\mathcal{N}_{1}}(\nu, w)=\mathbb{J}_{\mathcal{N}_{1}} \mathrm{D}_{w} h(\nu, w) .
\end{aligned}
$$

Here, $\mathcal{N}=\mathcal{N}_{0} \oplus \mathcal{N}_{1}$, and $v \in \mathcal{N}$ is decomposed as $v=(\nu, w), \nu \in \mathcal{N}_{0}, w \in \mathcal{N}_{1}$. The equation $\dot{v}=f_{\mathcal{N}}(v)$, where $f_{\mathcal{N}}=\left(f_{\mathcal{N}_{0}}, f_{\mathcal{N}_{1}}\right)$ is called the slice equation. The space $\mathcal{N}_{1}=\operatorname{ker} \operatorname{DJ}(\bar{x}) \cap \mathcal{N}$ is symplectic with symplectic structure matrix $\mathbb{J}_{\mathcal{N}_{1}}$ and is called symplectic normal space. Let $\mathbf{n}_{\bar{\mu}}$ be a $\Gamma_{\bar{\mu}}$-invariant complement to $\mathbf{g}_{\bar{\mu}}$ in $\mathbf{g}^{*}$. Then the annihilator $\operatorname{ann}_{\mathbf{g}^{*}}\left(\mathbf{n}_{\bar{\mu}}\right)$ of $\mathbf{n}_{\bar{\mu}}$ in $\mathbf{g}^{*}$ is a $\Gamma_{\bar{\mu}}$-invariant section transverse to the momentum group orbit $\Gamma \bar{\mu}$ at $\bar{\mu}$ in $\mathbf{g}^{*}$ and $\mathcal{N}_{0} \simeq \mathbf{g}_{\bar{\mu}}^{*} \simeq$ $\operatorname{ann}_{\mathbf{g}^{*}}\left(\mathbf{n}_{\bar{\mu}}\right)$. Moreover, $h(\nu, w)$ is the Hamiltonian in the coordinates $x \simeq(\gamma, \nu, w)$. The original relative equilibrium $\Gamma \bar{x}$ corresponds to the equilibrium $(\nu, w)=0$ of the slice equation, i.e., $\mathrm{D}_{w} h(0)=0$. Furthermore, we have $f_{\bar{\Gamma}}(0,0)=\bar{\xi}$, where $\bar{\xi}$ is the drift velocity of the relative equilibrium at $\bar{x}$. The momentum map in these coordinates takes the form

$$
\mathbf{j}(\gamma, \nu, w)=\gamma(\bar{\mu}+\nu) .
$$

Example 2.6 A simple example illustrating (2.5) is the spinning top without external forces [11]. In this case $\mathcal{X}=\Gamma \times \mathcal{N}$, we have $\Gamma=\mathrm{SO}(3), \mathcal{N}=\mathcal{N}_{0}=\mathrm{so}^{*}(3)$. Here $\gamma \in \mathrm{SO}(3)$ describes the angle of the top and $\nu \in \operatorname{so}(3)^{*} \simeq \mathbb{R}^{3}$ its angular momentum in body coordinates. In this case $h(\nu)=\frac{1}{2} \nu^{T} \mathbb{I}^{-1} \nu$ is the kinetic energy of the top, $\mathbb{I}$ its inertia tensor, and (2.5) becomes $\dot{\gamma}=\gamma \Omega, \dot{\nu}=\nu \times \Omega$ where $\Omega=\mathbb{I}^{-1} \nu$ is the instantaneous velocity of the top. Moreover $\bar{\mu}=0$ and (2.6) is the angular momentum of the spinning top in the inertial frame. 
With respect to the decomposition $\mathcal{T}_{\bar{x}} \mathcal{X}=\mathcal{T}_{\bar{x}} \Gamma \bar{x} \oplus \mathcal{N}_{0} \oplus \mathcal{N}_{1}$, the linearization $A=\mathrm{D}\left(f_{H}(\bar{x})-\bar{\xi}\right)$ at the relative equilibrium in a frame moving with its drift velocity $\bar{\xi}$ is

$$
A=\left(\begin{array}{ccc}
-\operatorname{ad}_{\bar{\xi}} & \mathrm{D}_{\nu}^{2} h(0) & \mathrm{D}_{\nu w}^{2} h(0) \\
0 & \left.\operatorname{ad}_{\xi}^{*}\right|_{\mathbf{g}_{\mu}^{*}} ^{*} & 0 \\
0 & \mathbb{J}_{\mathcal{N}_{1}} \mathrm{D}_{\nu w}^{2} h(0) & \mathbb{J}_{\mathcal{N}_{1}} \mathrm{D}_{w}^{2} h(0)
\end{array}\right),
$$

see [17]. We have

$$
\left.\operatorname{ker} \operatorname{ad}_{\bar{\xi}}\right|_{\mathbf{g}_{\bar{\mu}}} \oplus \operatorname{ad}_{\bar{\xi}} \mathbf{g}_{\bar{\mu}}=\mathbf{g}_{\bar{\mu}} .
$$

because $\Gamma$ is compact and $\operatorname{so~}_{\bar{\xi}}$ is semi-simple. Therefore

$$
\mathbf{g}_{\bar{\mu}}^{*}=\left.\left.\operatorname{kerad}\right|_{\bar{\xi}} ^{*}\right|_{\mathbf{g}_{\mu}^{*}} ^{*} \oplus \operatorname{ann}_{\mathbf{g}_{\bar{\mu}}^{*}}\left(\mathbf{g}_{(\bar{\xi}, \bar{\mu})}\right) \text {. }
$$

Here we use that $\left.\operatorname{ker} \operatorname{ad}_{\bar{\xi}}^{*}\right|_{\mathbf{g}_{\bar{\mu}}^{*}}=\operatorname{ann}_{\mathbf{g}_{\bar{\mu}}^{*}}\left(\operatorname{ad}_{\bar{\xi}} \mathbf{g}_{\bar{\mu}}\right)$ and $\mathbf{g}_{(\bar{\xi}, \bar{\mu})}=\left.\operatorname{ker} \operatorname{ad}_{\bar{\xi}}\right|_{\mathbf{g}_{\bar{\mu}}}$. Because of this we can identify

$$
\left.\operatorname{ker} \operatorname{ad}_{\bar{\xi}}^{*}\right|_{\mathbf{g}_{\bar{\mu}}^{*}} \simeq \mathbf{g}_{(\bar{\xi}, \bar{\mu})}^{*} .
$$

Decompose $\nu \in \mathbf{g}_{\bar{\mu}}^{*}$ as $\nu=\chi+\zeta$ where $\chi \in \mathbf{g}_{(\bar{\xi}, \bar{\mu})}^{*}$ and $\zeta \in \operatorname{ann}_{\mathbf{g}_{\bar{\mu}}^{*}}\left(\mathbf{g}_{(\bar{\xi}, \bar{\mu})}\right)$.

We see from (2.7) that $\left[A_{10}, A_{1}\right]$, where $A_{10}:=\mathbb{J}_{\mathcal{N}_{1}} \mathrm{D}_{\nu w}^{2} h(0)=\mathrm{D}_{\nu} f_{\mathcal{N}_{1}}(0), A_{1}:=\mathbb{J}_{\mathcal{N}_{1}} \mathrm{D}_{w}^{2} h(0)=$ $\mathrm{D}_{w} f_{\mathcal{N}_{1}}(0)$, may have full rank even if $A_{1}$ does not have full rank, due to Jordan blocks of $A$ to the eigenvalue 0 , which correspond to the kernel $\mathbf{g}_{(\bar{\xi}, \bar{\mu})}^{*}$ of $A_{0}:=\left.\operatorname{ad}_{\bar{\xi}}^{*}\right|_{\mathbf{g}_{\mu}^{*}}$ and the kernel of $A_{1}$. This motivates the following definition:

Definition 2.7 A relative equilibrium $\Gamma \bar{x}$ with regular velocity-momentum pair is called transversal if the matrix

$$
\left.\left[\mathrm{D}_{\chi} f_{\mathcal{N}_{1}}(\nu, w), \mathrm{D}_{w} f_{\mathcal{N}_{1}}(\nu, w)\right]\right|_{(\nu, w)=0}
$$

has full rank.

As in [19] we call a relative equilibrium non-degenerate if $\mathrm{D}_{w} f_{\mathcal{N}_{1}}(0)$ is invertible. Our definition of a transversal relative equilibrium is equivalent to the corresponding definition in [16]: By [16, Theorem 4] a relative equilibrium of a compact group action with regular velocity-momentum pair is transversal if in our notation the matrix $\mathrm{D}_{w} f_{\mathcal{N}_{1}}(0)$ is either invertible or has a semisimple eigenvalue 0 and if $\mathrm{D}_{\chi} f_{\mathcal{N}_{1}}(0), \chi \in \mathbf{g}_{(\bar{\xi}, \bar{\mu})}^{*}, \nu=(\chi, \zeta) \in \mathbf{g}_{\bar{\mu}}^{*}$, maps $\mathbf{g}_{(\bar{\xi}, \bar{\mu})}^{*}$ onto the kernel of $\mathrm{D}_{w} f_{\mathcal{N}_{1}}(0)$. Equation (5) of [16] gives, in our notation,

$$
A_{1} A_{10}=-A_{10} A_{0} .
$$

This implies that the image of $\mathrm{D}_{\chi} f_{\mathcal{N}_{1}}(0)$ lies in the kernel of $\mathrm{D}_{w} f_{\mathcal{N}_{1}}(0)$. Therefore, the definition of transversality in [16] is equivalent to Definition 2.7.

Remark 2.8 Let $\mathcal{S}^{\bar{\mu}}$ be a nonlinear slice transverse to the relative equilibrium $\Gamma \bar{x}$ in $\mathbf{J}^{-1}(\bar{\mu})$ with velocity-momentum pair $(\bar{\xi}, \bar{\mu})$. Then $\mathcal{T}_{\bar{x}} \mathcal{S}^{\bar{\mu}} \simeq \mathcal{N}_{1}, \mathcal{S}^{\bar{\mu}}$ is called the Marsden-Weinstein reduced phase space, and the relative equilibrium $\Gamma \bar{x}$ is a critical point of $\left.H\right|_{\mathcal{S}^{\bar{\mu}}}$, i.e., $\left.\mathrm{D} H\right|_{\mathcal{S}^{\bar{\mu}}}(\bar{x})=0$. If $\bar{\mu}$ is regular, then $\mathbf{g}_{(\bar{\xi}, \bar{\mu})}=\mathbf{g}_{\bar{\mu}}, \mathbf{g}_{\mu}$ has constant dimension for $\mu \approx \bar{\mu}$, and we can choose $\mathcal{S}^{\mu}$ to depend smoothly on $\mu$ and parametrize it by $w \in \mathcal{N}_{1}$. In this case the transversality condition from Definition (2.7) is equivalent to the condition that $\left.\mathrm{D}_{(\nu, w)} \mathrm{D} H\right|_{\mathcal{S}^{\bar{\mu}+\nu}}(\bar{x})$ has full rank. If the momentum $\bar{\mu}$ of the relative equilibrium is non-generic, then $\mathcal{S}_{\mu}$ changes dimension for $\mu \neq \bar{\mu}$, $\mu \approx \bar{\mu}$, and this equivalence does not hold.

As shown in [16], near a transversal relative equilibrium $\Gamma \bar{x}$ of a compact group action with regular velocity-momentum pair $(\bar{\xi}, \bar{\mu})$ there is an $r_{(\bar{\xi}, \bar{\mu})}$-dimensional manifold of relative equilibria: 
Theorem 2.9 Let $\bar{x}$ lie on a transversal relative equilibrium $\Gamma \bar{x}$ of (2.1) with regular velocitymomentum pair $(\bar{\xi}, \bar{\mu}) \in\left(\mathbf{g} \oplus \mathbf{g}^{*}\right)^{c}$. Let $r=r_{(\bar{\xi}, \bar{\mu})}$. Then there is an $r$-dimensional family $\Gamma x(s)$, $s \in \mathbb{R}^{r}$, of relative equilibria with momentum $\bar{\mu}+\chi(s), \chi(s) \in \mathbf{g}_{(\bar{\xi}, \bar{\mu})}^{*}$, and with drift velocity $\xi(s) \in \mathbf{g}_{(\bar{\xi}, \bar{\mu})}$ at $x(s)$ near $\Gamma \bar{x}$ such that $x(0)=\bar{x}, \chi(0)=0, \xi(0)=\bar{\xi}$. If the relative equilibrium $\Gamma \bar{x}$ is non-degenerate then we can choose $s=\chi \in \mathbf{g}_{(\bar{\xi}, \bar{\mu})}^{*}$.

Proof. For sake of completeness and to see the connection with the numerical continuation method of Section 2.1.2 we include a proof, which is different from the proof given in [16].

We see from (2.7) that $\bar{x}$ lies on a transversal relative equilibrium if we only reduce by the group $\tilde{\Gamma}=\Gamma_{\bar{\xi}}$. Denote by $\widetilde{\mathcal{N}}=\widetilde{\mathcal{N}}_{0} \oplus \widetilde{\mathcal{N}}_{1}$ a normal space transverse to the group orbit $\tilde{\Gamma} \bar{x}$ at $\bar{x}$ such that in the coordinates $x \simeq(\tilde{\gamma}, \tilde{\nu}, \tilde{w}), \tilde{\gamma} \in \tilde{\Gamma},(\tilde{\nu}, \tilde{w}) \in \tilde{\mathcal{N}}$, the vector-field (2.1) takes the form (2.5). Since, by Remark $2.4, \mathbf{g}_{(\bar{\xi}, \bar{\mu})}$ is abelian and since $\tilde{\nu}=\chi \in \widetilde{\mathcal{N}}_{0} \simeq \mathbf{g}_{(\bar{\xi}, \bar{\mu})}^{*}$, we have $f_{\widetilde{\mathcal{N}}_{0}}(\tilde{\nu}, \tilde{w}) \equiv 0$. Moreover, $\left[\mathrm{D}_{\tilde{\nu}} f_{\widetilde{\mathcal{N}}_{1}}(0), \mathrm{D}_{\tilde{w}} f_{\widetilde{\mathcal{N}}_{1}}(0)\right]$ has full rank. So there is a manifold of equilibria $(\tilde{\nu}(s), \tilde{w}(s))$ of $f_{\tilde{\mathcal{N}}}, s \in \mathbb{R}^{r}$, which gives a manifold of relative equilibria $\Gamma x(s)$ of $(2.1)$, with momentum $\mu(s) \mathbf{J}(x(s))=\bar{\mu}+\chi(s)$, where $x(s) \simeq(\mathrm{id}, \tilde{\nu}(s), \tilde{w}(s))$.

\subsubsection{Numerical computation of transversal relative equilibria}

It is well known that (relative) periodic orbits and relative equilibria of symmetric Hamiltonian systems can be computed numerically by adding unfolding parameters to overcome the degeneracies caused by conserved quantities, see e.g. [6, 14, 24]. In this section we extend results of [14] on continuation of relative equilibria to transversal relative equilibria of general compact group actions, see Remark 2.12 for a detailed comparison.

Let $\Gamma \bar{x}$ be a transversal relative equilibrium with regular velocity-momentum pair $(\bar{\xi}, \bar{\mu})$ at $\bar{x}$. Let $e_{\xi}^{1}, \ldots, e_{\xi}^{r}$ denote an orthonormal basis of $\mathbf{g}_{(\bar{\xi}, \bar{\mu})}$. Identify $\left(\xi_{1}, \ldots, \xi_{r}\right) \in \mathbb{R}^{r}$ with $\xi=\sum_{i=1}^{r} \xi_{i} e_{\xi}^{i}$ and set $\mathbf{J}_{i}(x):=\mathbf{J}_{e_{\xi}^{i}}(x)$. Because of Remark 2.4 generically the drift velocity $\bar{\xi}$ of the relative equilibrium is regular and we have $\mathbf{g}_{\bar{\xi}}=\mathbf{g}_{(\bar{\xi}, \bar{\mu})}$.

For a function $f: \mathcal{X} \rightarrow \mathbb{R}$ define $\nabla f(x)=(\mathrm{D} f(x))^{T} \in \mathcal{X}$. We show that the manifold of relative equilibria from Theorem 2.9 can be computed by solving the under-determined system of equations

$$
F\left(x, \xi, \lambda_{\mu}\right)=f_{H}(x)+\sum_{i=1}^{r} \lambda_{\mu, i} \nabla \mathbf{J}_{i}(x)-\sum_{i=1}^{r} \xi_{i} e_{\xi}^{i} x=0,
$$

with unfolding parameter $\lambda_{\mu} \in \mathbb{R}^{r}$ where $F: \mathcal{X} \times \mathbb{R}^{2 r} \rightarrow \mathbb{R}^{2 d}$ :

Theorem 2.10 Let $\bar{x}$ lie on a transversal relative equilibrium $\Gamma \bar{x}$ with regular velocity $\bar{\xi} \in \mathbf{g}$ and momentum $\bar{\mu}$ and let $r=r_{(\bar{\xi}, \bar{\mu})}$. Then the derivative $\mathrm{D} F(y)$ of (2.10) has full rank at any solution $y=\left(x, \xi, \lambda_{\mu}\right)$ of $F=0$ close to $(\bar{x}, \xi, 0)$, and any such solution satisfies $\lambda_{\mu}=0$ and, hence, determines a relative equilibrium of (2.1).

Proof. From (2.7) we see that, to take account of the symmetry-induced kernel vectors of $A=\mathrm{D}\left(f_{H}(\bar{x})-\bar{\xi}\right)$, it suffices to reduce by $\widetilde{\Gamma}:=\Gamma_{\bar{\xi}}$, as in the proof of Theorem 2.9. Moreover,

$$
\mathrm{D} F(\bar{x}, \bar{\xi}, 0)=\left(\mathrm{D}\left(f_{H}(\bar{x})-\bar{\xi}\right),-e_{\xi}^{1} \bar{x}, \ldots,-e_{\xi}^{r} \bar{x}, \nabla \mathbf{J}_{1}(\bar{x}), \ldots, \nabla \mathbf{J}_{r}(\bar{x})\right),
$$

has full rank if and only if $\left[\mathrm{D}_{\tilde{\nu}} f_{\widetilde{\mathcal{N}}_{1}}(0), \mathrm{D}_{\tilde{w}} f_{\widetilde{\mathcal{N}}_{1}}(0)\right]$ has image $\widetilde{\mathcal{N}}_{1}$, with the notation from the proof of Theorem 2.9. This condition is satisfied if and only if the relative equilibrium is transversal.

The solution manifold of (2.10) has dimension $2 r$. The points $(\gamma x(s), \xi(s), 0)$, where $\gamma \in$ $\Gamma_{(\bar{\xi}, \bar{\mu})}^{\mathrm{id}}$, and $x(s), \xi(s)$ as in Theorem 2.9 , are solutions of $F(x, \xi, 0)=0$, with $F$ from $(2.10)$, and 
this set is also $2 r$-dimensional. Therefore we have $\lambda_{\mu}=0$ in any solution of $(2.10)$ near $(\bar{x}, \bar{\xi}, 0)$.

Under the assumptions of Theorem 2.10, the under-determined system (2.10) is amenable to standard numerical methods; for example, the Gauss-Newton method applied to (2.10) converges for initial values $y=\left(x, \xi, \lambda_{\mu}\right)$ close to $(\bar{x}, \bar{\xi}, 0)$.

Remark 2.11 In Theorem 2.9 we only assume that the relative equilibrium $\Gamma \bar{x}$ has a regular velocity-momentum pair $(\bar{\xi}, \bar{\mu})$ and not necessarily a regular velocity $\bar{\xi}$, so that in general $\mathbf{g}_{\bar{\xi}} \neq$ $\mathbf{g}_{(\bar{\xi}, \bar{\mu})}$. In this case let $e_{\xi}^{1}, \ldots, e_{\xi}^{q}$ denote an orthonormal basis of $\mathbf{g}_{\bar{\xi}}$ such that $\operatorname{span}\left(e_{\xi}^{r+1}, \ldots, e_{\xi}^{q}\right)=$ $\mathbf{n}_{\bar{\mu}} \cap \mathbf{g}_{\bar{\xi}}$ and $\operatorname{span}\left(e_{\xi}^{1}, \ldots, e_{\xi}^{r}\right)=\mathbf{g}_{(\bar{\xi}, \bar{\mu}}$. As before, identify $\xi=\left(\xi_{1}, \ldots, \xi_{q}\right) \in \mathbb{R}^{q}$ with $\sum_{i=1}^{q} \xi_{i} e_{\xi}^{i}$, and set $\mathbf{J}_{j}(x):=\mathbf{J}_{e_{\xi}^{j}}(x)$; for $\mu \in \mathbf{g}^{*}$ let $\mu_{j}=\mu\left(e_{\xi}^{j}\right), j=1, \ldots, q$.

Because of Remark 2.4 we generically have $q=r$. In the case that $q>r$ the derivative $\mathrm{D} F(\bar{x}, \bar{\xi}, 0)$ of $(2.10)$ does not have full rank, and the convergence of numerical methods to solve (2.10) is expected to be slow, also at relative equilibria nearby. Instead of (2.10) it is then advantageous to solve

$$
F\left(x, \xi, \lambda_{\mu}\right)=\left(\begin{array}{c}
f_{H}(x)+\sum_{i=1}^{r} \lambda_{\mu, i} \nabla \mathbf{J}_{i}(x)-\sum_{i=1}^{q} \xi_{i} e_{\xi}^{i} x \\
\mathbf{J}_{r+1}(x)-\bar{\mu}_{r+1} \\
\vdots \\
\mathbf{J}_{q}(x)-\bar{\mu}_{q}
\end{array}\right)=0,
$$

where $F: \mathcal{X} \times \mathbb{R}^{q} \times \mathbb{R}^{r} \rightarrow \mathbb{R}^{2 d} \times \mathbb{R}^{q-r}$. Note that $\bar{\mu}_{j}=0$ for $j=r+1, \ldots, q$ since $\bar{\mu} \in \mathbf{g}_{\bar{\xi}}^{*}$ and we identify adjoint and co-adjoint actions. Because of (2.7) the derivative of (2.11)

$$
\mathrm{D} F(\bar{x}, \bar{\xi}, 0)=\left(\begin{array}{ccccccc}
\mathrm{D}\left(f_{H}(\bar{x})-\bar{\xi}\right) & -e_{\xi}^{1} \bar{x} & \ldots & -e_{\xi}^{q} \bar{x} & \nabla \mathbf{J}_{1}(\bar{x}) & \ldots & \nabla \mathbf{J}_{r}(\bar{x}) \\
\mathrm{D} \mathbf{J}_{r+1}(\bar{x}) & 0 & \ldots & 0 & 0 & \ldots & 0 \\
\vdots & 0 & \ldots & 0 & 0 & \ldots & 0 \\
\mathrm{D} \mathbf{J}_{q}(\bar{x}) & 0 & \ldots & 0 & 0 & \ldots & 0
\end{array}\right)
$$

has full rank, and the solution manifold of (2.11) is $2 r$-dimensional. Moreover, all solutions close to the original relative equilibrium satisfy $\xi_{i}=0, i=r+1, \ldots, q$. To see this note that, as in the proof of Theorem 2.10, the points $(\gamma x(s), \xi(s), 0,0), s \in \mathbb{R}^{r}$, where $x(s), \xi(s)$ from Theorem $2.9, \gamma \in \Gamma_{(\bar{\xi}, \bar{\mu}}^{\mathrm{id}}$, form a $2 r$-dimensional manifold of solutions of (2.11) as their momentum values $\mu(s)=\mathbf{J}(x(s))$ satisfy $\mu(s)-\bar{\mu} \in \mathbf{g}_{(\bar{\xi}, \bar{\mu})}^{*} \subseteq \operatorname{ann}\left(\mathbf{n}_{\bar{\mu}}\right)$ and hence $\mu_{j}(s)=\bar{\mu}_{j}, j=r+1, \ldots q$.

Remark 2.12 Muñoz-Almaraz et al. study relative equilibria of Hamiltonian systems (2.1) under the assumption that $\Gamma^{\text {id }}$ is abelian. They call a relative equilibrium $\Gamma \bar{x}$ of $(2.1)$ with drift velocity $\bar{\xi}$ normal if in our notation

$$
\operatorname{range}\left(\mathrm{D}\left(f_{H}(\bar{x})-\bar{\xi}\right)\right)+\mathbf{g} \bar{x}=\operatorname{ker} \mathrm{D} \mathbf{J}(\bar{x})
$$

see equation (6.9) of [14]. For abelian $\Gamma^{\text {id }}$ they prove in [14, Theorem 16] that a normal relative equilibrium $\Gamma \bar{x}$ persists to a $g$-dimensional manifold of points $x$ on relative equilibria which lie in a section $\mathcal{N}$ transverse to $\Gamma \bar{x}$ at $\bar{x}$. Here $g=\operatorname{dim} \Gamma$. By projecting (2.12) to $\mathcal{N}_{1}$ we see that relative equilibria which are normal in the above sense are also transversal. In this case Theorem 2.9 applies $r_{(\bar{\xi}, \bar{\mu})}=g$. Muñoz-Almaraz et al. also show that this manifold of relative equilibria can be computed numerically by solving a system of the form (2.10), with (in our notation) $\xi \in \mathbf{g}, \lambda_{\mu} \in \mathbf{g}^{*} \simeq \mathbb{R}^{g}$, and with $g$ phase conditions, determining a section $\mathcal{N}$ transverse to the group orbit. We decide not to fix the phase of the relative equilibria which we compute and rather to solve the under-determined system (2.10); apart from this minor difference, our Theorem 2.10 extends their numerical method to general compact symmetry groups. 


\subsubsection{Numerical path-following of relative equilibria}

Let, as before, $\bar{x}$ lie on a transversal relative equilibrium $\Gamma \bar{x}$ with regular velocity-momentum pair $(\bar{\xi}, \bar{\mu})$. Let $\left\{e_{\xi}^{i}, i=1, \ldots, g\right\}$ be an orthonormal basis of $\mathbf{g}$ such that $\operatorname{span}\left\{e_{\xi}^{i}, i=q+1, \ldots, g\right\}=$ $\operatorname{ad}_{\bar{\xi}} \mathbf{g}, \operatorname{span}\left\{e_{\xi}^{i}, i=1, \ldots, q\right\}=\mathbf{g}_{\bar{\xi}}, \operatorname{span}\left\{e_{\xi}^{i}, i=1, \ldots, r\right\}=\mathbf{g}_{(\bar{\xi}, \bar{\mu})}$, c.f. (2.8). For $\mu \in \mathbf{g}^{*}$ let, as before, $\mu_{j}=\mu\left(e_{\xi}^{j}\right), j=1, \ldots, g$. We fix $g-1$ momentum components, without loss of generality, the components $\mu^{\mathrm{b}}=\left(\mu_{2}, \ldots \mu_{g}\right), g=\operatorname{dim} \Gamma$, to get a one-parameter family. We solve

$$
F^{\bar{\mu}^{b}}\left(x, \xi, \lambda_{\mu}\right):=\left(\begin{array}{c}
F\left(x, \xi, \lambda_{\mu}\right) \\
\mathbf{J}_{2}(x)-\bar{\mu}_{2} \\
\vdots \\
\mathbf{J}_{r}(x)-\bar{\mu}_{r}
\end{array}\right)=0 .
$$

Then for any solution $y=(x, \xi, 0)$ of $(2.13)$ the $x$-component lies in

$$
\mathcal{X}^{\mu^{b}}=\left\{x \in \mathcal{X}, \quad \mathbf{J}_{j}(x)=\bar{\mu}_{j}, j=2, \ldots, g\right\} .
$$

To see this, first note that for $\xi \in \mathbf{g}_{\bar{\xi}}$ with $\xi \approx \bar{\xi}$ we have $\mathbf{g}_{\xi} \subseteq \mathbf{g}_{\bar{\xi}}, \mathbf{g}_{\xi}^{*} \subseteq \mathbf{g}_{\bar{\xi}}^{*}$. Here, as in (2.9), we identify $\mathbf{g}_{\xi}^{*}=\operatorname{ker} \operatorname{ad}_{\xi}^{*}$. By construction $\xi \in \mathbf{g}_{\bar{\xi}}$ at any solution $y=(x, \xi, 0)$ of $(2.13)$. As stated in (2.3), we have $\operatorname{ad}_{\xi}^{*} \mu=0$ or, equivalently, $\mu \in \mathbf{g}_{\xi}^{*}$ for the drift velocity $\xi$ and momentum $\mu=\mathbf{J}(x)$ of the relative equilibrium given by $y=(x, \xi, 0)$. From this we conclude that $\mathbf{J}(x) \in \mathbf{g}_{\xi}^{*}$ and, thus, $\mathbf{J}_{j}(x)=0=\bar{\mu}_{j}, j=q+1, \ldots, g$, whenever $(x, \xi, 0)$ solves (2.13). If $q \neq r$ then $\mu_{j}=\bar{\mu}_{j}$, $j=r+1, \ldots, q$ are sub-equations of $F=0$, see (2.11). Hence, the solutions of (2.13) satisfy $\mathbf{J}^{\mathrm{b}}(x)=\bar{\mu}^{\mathrm{b}}$. Moreover, we have:

Corollary 2.13 Let $\Gamma \bar{x}$ be a transversal relative equilibrium with regular velocity-momentum pair $(\bar{\xi}, \bar{\mu})$ and let $\chi(s)$ as in Theorem 2.9. Then the $(r-1, r)$-matrix $\left.\partial_{s}\left(\chi_{2}, \ldots, \chi_{r}\right)(s)\right|_{s=0}$ has full rank if and only if $\left[\mathrm{D}_{\chi_{1}} f_{\mathcal{N}_{1}}(0), \mathrm{D}_{w} f_{\mathcal{N}_{1}}(0)\right]$ has full rank and if and only if $\mathrm{D} F^{\bar{\mu}^{b}}(\bar{x}, \bar{\xi}, 0)$ has full rank. In this case there is a path $x(\epsilon)$ of points on relative equilibria in $\mathcal{X}^{\bar{\mu}^{b}}$ with drift velocity $\xi(\epsilon)$ such that $x(0)=\bar{x}, \xi(0)=\bar{\xi}$, and $(x(\epsilon), \xi(\epsilon), 0)$ solves $(2.13)$.

If the matrix $\left[\mathrm{D}_{\chi_{1}} f_{\mathcal{N}_{1}}(0), \mathrm{D}_{w} f_{\mathcal{N}_{1}}(0)\right]$ above has full rank we say that the relative equilibrium $\Gamma \bar{x}$ is transversal with respect to $C(x):=\mathbf{J}_{1}(x)$.

Under the above assumption, (2.13) can be solved by standard numerical methods for underdetermined systems, for example by the Gauss-Newton method, for initial values close to $\bar{y}=$ $(\bar{x}, \bar{\xi}, 0)$. For tangential continuation methods, we choose a nontrivial continuation tangent $t=t(\bar{y})$ in a solution point $\bar{y}$ in the kernel of $\mathrm{D} F^{\bar{\mu}^{b}}(\bar{y})$, which is orthogonal to the group orbit, i.e., $\left\langle t, t^{\xi_{i}}\right\rangle=0, i=1, \ldots, r$. Here $t^{\xi_{i}} \in \operatorname{ker}\left(\mathrm{D} F^{\bar{\mu}^{b}}(\bar{y})\right)$ is given by $t^{\xi_{i}}=\left(t_{x}^{\xi_{i}}, t_{\xi}^{\xi_{i}}, t_{\lambda_{\mu}}^{\xi_{i}}\right), t_{x}^{\xi_{i}}=e_{\xi}^{i} \bar{x}$, $t_{\xi}^{\xi_{i}}=0, t_{\lambda_{\mu}}^{\xi_{i}}=0, i=1, \ldots, r$.

\subsection{Persistence of transversal RPOs}

A point $\bar{x} \in \mathcal{X}$ lies on a relative periodic orbit (RPO) if there exists $t>0$ such that $\Phi^{t}(\bar{x}) \in \Gamma \bar{x}$. The infimum $\bar{\tau}$ of such $t$ is called the relative period of the RPO and the element $\bar{\sigma} \in \Gamma$ such that $\bar{\sigma} \Phi^{\bar{\tau}}(\bar{x})=\bar{x}$ is called the reconstruction phase or drift symmetry of the RPO. The relative periodic orbit $\overline{\mathcal{P}}$ itself is given by

$$
\overline{\mathcal{P}}=\left\{\gamma \Phi^{\theta}(\bar{x}), \gamma \in \Gamma, \theta \in \mathbb{R}\right\} .
$$

Throughout the paper we assume that the RPOs which we consider are proper, i.e., not relative equilibria, and that, without loss of generality, the isotropy $K$ of the RPO is trivial (if not, we 
restrict the dynamics to the fixed point space $\operatorname{Fix}(K)$ of $K$ and replace $\Gamma$ by $N(K)$ so that $K$ is trivial). For $\alpha \in \Gamma, \xi \in \mathbf{g}$ let $Z(\alpha)=\{\gamma \in \Gamma, \quad \gamma \alpha=\alpha \gamma\}$ and $Z(\xi)=\left\{\gamma \in \Gamma, \operatorname{Ad}_{\gamma} \xi=\xi\right\}$ denote the centralizer of $\alpha$ and $\xi$, respectively.

An RPO of a compact group action becomes periodic in a co-moving frame as the following lemma shows:

Lemma 2.14 [21, 20, 24]

a) Any element $\sigma$ of a compact group $\Gamma$ can be decomposed as

$$
\sigma=\alpha \exp (-\xi)
$$

for some $\xi \in \mathbf{g}$ and $\alpha \in \Gamma$ such that

$$
\alpha^{\ell}=\text { id } \text { for some } \ell \in \mathbb{N}, \quad \operatorname{Ad}_{\alpha} \xi=\xi,
$$

and

$$
Z(\sigma)=Z(\alpha) \cap Z(\xi) .
$$

b) For any RPO with drift-momentum pair $(\bar{\sigma}, \bar{\mu}) \in\left(\Gamma \times \mathbf{g}^{*}\right)^{c}$ and relative period $\bar{\tau}$ there is a frame moving with velocity $\bar{\xi} \in \mathbf{g}_{(\bar{\sigma}, \bar{\mu})}$, called drift velocity of the RPO with respect to $\bar{x}$, and some integer $\ell$ such that in this co-moving frame the RPO becomes a periodic orbit of period $\bar{T}=\ell \bar{\tau}$ and has the drift symmetry $\alpha \in \Gamma_{\bar{\mu}}$. Moreover, $\bar{\sigma}=\alpha \exp (-\bar{\tau} \bar{\xi})$.

As usual (see [7]), we call the group of elements $\gamma \in \Gamma$ which leave a periodic orbit invariant its spatio-temporal symmetry group. In a comoving frame, the RPO from Lemma $2.14 \mathrm{~b}$ ) above is a periodic orbit with spatio-temporal symmetry group $\mathbb{Z}_{\ell}(\alpha)$. Here $\mathbb{Z}_{\ell}(\alpha)$ denotes the group generated by the element $\alpha$ of order $\ell$.

Remark 2.15 Note that the decomposition (2.14) is in general not unique: assume that the group $C$ generated by $\sigma$ is continuous. Let $\eta$ be an infinitesimal rotation in the Lie algebra of $C$ which generates the rotation group $\exp (\phi \eta)=R_{\phi} \in C, \phi \in[0,2 \pi]$. Then other possible choices for $\alpha$ and $\xi$ would be $\hat{\alpha}=R_{2 \pi j / \ell} \alpha$ where $j \in \mathbb{Z}, \operatorname{gcd}(\ell, j)=1$, and $\hat{\xi}=\xi+2 \pi\left(n+\frac{j}{\ell}\right) \eta, n \in \mathbb{N}$.

Let $\bar{x}$ lie on an RPO with drift symmetry $\bar{\sigma}$, momentum $\bar{\mu}=\mathbf{J}(\bar{x})$ at $\bar{x}$, and relative period $\bar{\tau}$. The relation between the drift symmetry and momentum of an RPO

$$
\bar{\sigma} \bar{\mu}=\bar{\mu},
$$

is analogous to the corresponding relation (2.3) for relative equilibria, and is crucial for the problem of persistence to nearby momentum values, see [20] and the sections below. It is a consequence of the fact that $\mathbf{J}$ is preserved by the flow, and so $\bar{\sigma} \bar{\mu}=\bar{\sigma} \mathbf{J}(\bar{x})=\mathbf{J}(\bar{\sigma} \bar{x})=$ $\mathbf{J}\left(\Phi^{-\bar{\tau}}(\bar{x})\right)=\mathbf{J}(\bar{x})=\bar{\mu}$.

We need the following definitions, which are analogous to the corresponding Definition 2.3 for relative equilibria.

Definition 2.16 [20], [24]

(i) We call pairs $(\sigma, \mu) \in \Gamma \times \mathbf{g}^{*}$ satisfying (2.15) drift-momentum pairs and denote the space of drift-momentum pairs by

$$
\left(\Gamma \times \mathbf{g}^{*}\right)^{c}:=\left\{(\sigma, \mu) \in \Gamma \times \mathbf{g}^{*}, \quad \sigma \mu=\mu\right\} .
$$


(ii) We define an action of $\Gamma$ on the space of drift-momentum pairs as follows:

$$
\gamma(\sigma, \mu)=\left(\gamma \sigma \gamma^{-1},\left(\operatorname{Ad}_{\gamma}^{*}\right)^{-1} \mu\right), \quad \text { for } \gamma \in \Gamma,(\sigma, \mu) \in\left(\Gamma \times \mathbf{g}^{*}\right)^{c} .
$$

For later purposes we denote the isotropy subgroup of $(\sigma, \mu) \in\left(\Gamma \times \mathbf{g}^{*}\right)^{c}$ with respect to this action by $\Gamma_{(\sigma, \mu)}$, its Lie algebra by $\mathbf{g}_{(\sigma, \mu)}$ and set $r_{(\sigma, \mu)}=\operatorname{dim} \mathbf{g}_{(\sigma, \mu)}$. Moreover, we define the isotropy subgroup of $\sigma \in \Gamma$ as $\Gamma_{\sigma}=\Gamma_{(\sigma, 0)}$, denote its Lie algebra by $\mathbf{g}_{\sigma}$ and let $r_{\sigma}=\operatorname{dim} \mathbf{g}_{\sigma}$.

(iii) We call a drift-momentum pair $(\sigma, \mu) \in\left(\Gamma \times \mathbf{g}^{*}\right)^{c}$ regular if $r_{(\sigma, \mu)}$ is locally constant in the space of drift-momentum pairs (2.16).

(iv) We call $\sigma \in \Gamma$ regular if $r_{\sigma}$ is locally constant.

Remark 2.17 [20, 24] Regular drift-momentum pairs $(\sigma, \mu)$ are generic in the space of driftmomentum pairs $\left(\Gamma \times \mathbf{g}^{*}\right)^{c}$. Regular elements $\sigma \in \Gamma$ are generic in $\Gamma$. The drift symmetry $\sigma$ of a regular drift-momentum pair $(\sigma, \mu)$ is generically regular in which case $\mathbf{g}_{\sigma}=\mathbf{g}_{(\sigma, \mu)}$, and generically the momentum $\mu$ of a regular drift-momentum pair $(\sigma, \mu)$ is regular in which case $\mathbf{g}_{\mu}=\mathbf{g}_{(\sigma, \mu)}$. Moreover, the following conditions are equivalent:

(i) $(\sigma=\alpha \exp (-\xi), \mu)$ is a regular drift-momentum pair;

(ii) $\mathbf{g}_{(\sigma, \mu)}$ is the Lie algebra of a Cartan subgroup (for a definition of the term Cartan subgroup see, e.g., [2]);

(iii) $\mathbf{g}_{(\sigma, \mu)}=\mathbf{g}_{\alpha} \cap \mathbf{g}_{(\xi, \mu)}$ is abelian.

Let $\bar{x}$ lie on an RPO $\overline{\mathcal{P}}$ with relative period $\bar{\tau}$. We call a section $\mathcal{S}:=\mathcal{S}_{\bar{x}}$ that is transverse to the RPO at $\bar{x}$, i.e., transverse to $\mathbf{g} \bar{x} \oplus \operatorname{span}\left(f_{H}(\bar{x})\right)$, a Poincaré section at $\bar{x}$. As usual, we define the Poincaré map $\Pi: \mathcal{S} \rightarrow \mathcal{S}$ as follows. For $x \in \mathcal{S}$ close to $\bar{x}$ there are unique $\gamma(x) \in \Gamma$, $\gamma(x) \approx \bar{\sigma}$, and $\tau(x) \approx \bar{\tau}$ such that $\gamma(x) \Phi^{\tau(x)}(x) \in \mathcal{S}$ (this follows from the implicit function theorem since we assume that the isotropy $K$ of the RPO is trivial). Now we set

$$
\Pi(x)=\gamma(x) \Phi^{\tau(x)}(x) .
$$

Let $\bar{E}=H(\bar{x})$ and $\bar{\mu}=\mathbf{J}(\bar{x})$ be the energy and momentum of the RPO at $\bar{x}$ and let $\mathcal{S}^{\bar{E}, \bar{\mu}} \subseteq \mathcal{X}^{\bar{E}, \bar{\mu}}$ be a Poincaré-section transverse to the time orbit and $\Gamma_{\bar{\mu}}$-orbit through $\bar{x}$ within the energy momentum level set

$$
\mathcal{X}^{\bar{E}, \bar{\mu}}=\{x \in \mathcal{X}, \quad \bar{E}=H(x), \quad \bar{\mu}=\mathbf{J}(x)\} .
$$

Denote the corresponding Poincaré-map by $\Pi^{\bar{E}, \bar{\mu}}: \mathcal{S}^{\bar{E}, \bar{\mu}} \rightarrow \mathcal{S}^{\bar{E}, \bar{\mu}}$. For the definition of transversality of an RPO we need the following lemma:

Lemma 2.18 There is a choice of Poincaré-section $\mathcal{S}$ near an RPO at $\bar{x}$ with momentum $\bar{\mu}=\mathbf{J}(\bar{x})$, energy $\bar{E}$ and drift symmetry $\bar{\sigma}$, such that $\mathcal{S}^{\bar{E}, \bar{\mu}}=\mathcal{S} \cap \mathcal{X}^{\bar{E}, \bar{\mu}}$ and the following hold true:

a) The tangent space $\mathcal{T}_{\bar{x}} \mathcal{S}$ to the Poincaré-section $\mathcal{S}$ at $\bar{x}$ takes the form

$$
\mathcal{T}_{\bar{x}} \mathcal{S} \simeq \mathcal{N}=\mathcal{N}_{0} \oplus \mathcal{N}_{1} \oplus \mathcal{N}_{2}
$$

where

$$
\mathcal{N}_{0} \simeq \mathbf{g}_{\bar{\mu}}^{*}, \quad \mathcal{N}_{1} \simeq \mathcal{T}_{\bar{x}} \mathcal{S} \cap \operatorname{kerDJ}(\bar{x}) \cap \operatorname{kerDH}(\bar{x}), \quad \mathcal{N}_{2} \simeq \mathbb{R}
$$

Here, $\mathcal{N}_{1}=\mathcal{T}_{\bar{x}} \mathcal{S}^{\bar{E}, \bar{\mu}}$ is a symplectic vector-space, $\mathcal{N}_{0}$ is isomorphic to a $\Gamma_{\bar{\mu}}$-invariant section transverse to the momentum group orbit $\Gamma \bar{\mu}$ in $\mathbf{g}^{*}$ and $\mathcal{N}_{2}$ parametrizes the energy level. 
b) Let $\Pi: \mathcal{S} \rightarrow \mathcal{S}$ be the Poincaré map. Then there is a choice of coordinates $x \simeq v=$ $(\nu, w, E)$, for $x \in \mathcal{S}$, such that $v \in \mathcal{N}, \nu \in \mathcal{N}_{0}, w \in \mathcal{N}_{1}$ and $E \in \mathcal{N}_{2}$, the point $\bar{x}$ is identified with $v=(\nu, w, E)=(0,0, \bar{E})$, and the Poincaré map takes the form

$$
\Pi(\nu, w, E)=\left(\Pi_{\mathcal{N}_{0}}(\nu, w, E), \Pi_{\mathcal{N}_{1}}(\nu, w, E), \Pi_{\mathcal{N}_{2}}(\nu, w, E)\right) .
$$

Here,

$$
\Pi_{\mathcal{N}_{0}}(\nu, w, E)=\gamma(\nu, w, E) \nu, \Pi_{\mathcal{N}_{2}}(\nu, w, E)=E, \quad \Pi_{\mathcal{N}_{1}}(0, w, \bar{E})=\Pi^{\bar{E}, \bar{\mu}}(w),
$$

$\gamma(\nu, w, E) \in \Gamma_{\bar{\mu}}, \gamma(0,0, \bar{E})=\bar{\sigma}$, and the map $w \rightarrow \Pi_{\mathcal{N}_{1}}(\nu, w, E)$ is symplectic. In these coordinates the momentum map restricted to $\mathcal{N}$ takes the form

$$
\left.\mathbf{j}\right|_{\mathcal{N}}(\nu, w)=\bar{\mu}+\nu .
$$

Proof. Part a) is contained in [22, Theorem 3.1] and most of part b) is implicitly contained in [20]:

Theorem 3.1 and Theorem 6.9 of [22] imply that a $\Gamma$-invariant neighbourhood $\mathcal{U}$ of the RPO $\overline{\mathcal{P}}$ through $\bar{x}$ is symplectomorphic to $(\Gamma \times \mathbb{R} / 2 \pi \mathbb{Z} \times \mathcal{N}) / \mathbb{Z}_{\ell}$ where $\mathcal{N}$ can be decomposed as specified above. The $\mathbb{Z}_{\ell}$-action is generated by the drift symmetry $\alpha$ of the RPO $\overline{\mathcal{P}}$ in the co-moving frame and acts as $(\gamma, v, \theta) \rightarrow\left(\gamma \alpha^{-1}, Q v, \theta+1\right)$. Here $Q(\nu, w, E)=\left(\operatorname{Ad}_{\alpha}^{*} \nu, Q_{\mathcal{N}_{1}} w, e\right)$ for $v=(\nu, w, e) \in \mathcal{N}=\mathcal{N}_{0} \oplus \mathcal{N}_{1} \oplus \mathcal{N}_{2}$, and $Q_{\mathcal{N}_{1}}$ has order $\ell$. The symplectic form is $\mathbb{Z}_{\ell \text {-invariant }}$ and given by

$$
\Omega_{\Gamma \times \mathcal{N} \times \mathcal{T}^{*} S^{1}}=\Omega_{\Gamma \times \mathbf{g}_{\mu}^{*}}+\Omega_{\mathcal{N}_{1}}+\Omega_{\mathcal{T}^{*} S^{1}},
$$

on $\Gamma \times \mathbb{R} / 2 \pi \mathbb{Z} \times \mathcal{N}$. Here, $\mathcal{T}^{*} S^{1} \simeq \mathbb{R} / 2 \pi \times \mathcal{N}_{2}, \Omega_{\mathcal{T}^{*} S^{1}}$ is the symplectic form on $\mathcal{T}^{*} S^{1}, \Omega_{\Gamma \times \mathbf{g}_{\mu}^{*}}$ is the restriction to $\Gamma \times \mathbf{g}_{\bar{\mu}}^{*} \simeq \Gamma \times \operatorname{ann}_{\mathbf{g}^{*}}\left(\mathbf{n}_{\bar{\mu}}\right) \subseteq \Gamma \times \mathbf{g}^{*} \simeq \mathcal{T}^{*} \Gamma$ of the symplectic form on $\mathcal{T}^{*} \Gamma$, and $\Omega_{\mathcal{N}_{1}}$ is the symplectic form on $\mathcal{N}_{1}$. As before, $\mathbf{n}_{\bar{\mu}}$ is a $\Gamma_{\bar{\mu}}$-invariant complement to $\mathbf{g}_{\bar{\mu}}$ in $\mathbf{g}$. The energy of $(\gamma, \nu, w, e, \theta)$ is $E=h(\nu, w, \theta)+e$ and its momentum is $\mathbf{J}(\gamma, \nu, w, e, \theta)=\gamma(\bar{\mu}+\nu)$, see $[22$, Remark 3.4d),e)]. In the coordinates $(\gamma, \nu, w, E, \theta)$ the Poincaré-section $\mathcal{S}$ is given by $\mathcal{S}=\{(\gamma, \nu, w, E, \theta) \in(\Gamma \times \mathcal{N}) / \mathbb{Z}, \quad \theta=0\}$, and the Poincaré-map $\Pi(x)=\gamma(x) \Phi^{\tau(x)}(x)$ becomes a map $\Pi: \mathcal{N} \rightarrow \mathcal{N}$ which decomposes into $\Pi=\left(\Pi_{0}, \Pi_{1}, \Pi_{2}\right)$, where $\Pi_{0}$ maps into $\mathcal{N}_{0}, \Pi_{1}$ into $\mathcal{N}_{1}$ and $\Pi_{2}$ into $\mathcal{N}_{2}$. Due to the form of the momentum map in these coordinates and the conservation of momentum we have $\Pi_{0}(\nu, w, E)=\gamma(\nu, w, E)(\bar{\mu}+\nu)-\bar{\mu}$. Since $\operatorname{ann}\left(\mathbf{n}_{\bar{\mu}}\right)$ is $\Gamma_{\bar{\mu}}$-invariant and $\nu \in \operatorname{ann}\left(\mathbf{n}_{\bar{\mu}}\right), \nu \approx 0$, we have $\gamma(\nu, w, E) \in \Gamma_{\bar{\mu}}$ and, thus, $\Pi_{0}(\nu, w, E)=\gamma(\nu, w, E) \nu$. Energy conservation implies that $\Pi_{2}(\nu, w, E) \equiv E$. Moreover, due to the form of the symplectic form (2.18), the map $\Pi_{1}(\nu, \cdot, E): \mathcal{N}_{1} \rightarrow \mathcal{N}_{1}$ is symplectic.

The relation between the linearization of the Poincaré-map and the full linearization $\mathrm{D}_{x} \bar{\sigma} \Phi^{\bar{\tau}}(\bar{x})$ at the RPO through $\bar{x}$ is given in

Proposition 2.19 [22, Propositions 4.3 and 4.4] Let $\bar{x}=\bar{\sigma} \Phi^{\bar{\tau}}(\bar{x}) \in \mathcal{X}$ lie on an RPO $\overline{\mathcal{P}}$ of a compact group action $\Gamma$ with relative period $\bar{\tau}$ and momentum $\bar{\mu}=\mathbf{J}(\bar{x})$. Then the matrix $M=\mathrm{D}_{x} \bar{\sigma} \Phi^{\bar{\tau}}(\bar{x})$ has the structure

$$
M=\left(\begin{array}{ccc}
\operatorname{Ad}_{\bar{\sigma}} & 0 & D_{\mathcal{N}} \\
0 & 1 & \Theta_{\mathcal{N}} \\
0 & 0 & M_{\mathcal{N}}
\end{array}\right),
$$

with respect to the decomposition $\mathcal{T}_{\bar{x}} \mathcal{X}=\mathcal{T}_{\bar{x}} \Gamma \bar{x} \oplus \operatorname{span}\left(f_{H}(\bar{x})\right) \oplus \mathcal{N}$. The block $M_{\mathcal{N}}=\mathrm{D} \Pi(\bar{x})$ in (2.19) has the structure

$$
M_{\mathcal{N}}=\left(\begin{array}{ccc}
M_{0} & 0 & 0 \\
M_{10} & M_{1} & M_{1,2} \\
0 & 0 & 1
\end{array}\right)
$$


where $M_{0}=\left.\operatorname{Ad}_{\bar{\sigma}^{-1}}^{*}\right|_{\mathbf{g}_{\mu}^{*}}$, with respect to the decomposition $\mathcal{N}=\mathcal{N}_{0} \oplus \mathcal{N}_{1} \oplus \mathcal{N}_{2}$, and $\Theta_{\mathcal{N}}=$ $\left(\Theta_{0}, \Theta_{1}, \Theta_{2}\right)$. Moreover, with respect to the decompositions $\mathbf{g}=\mathbf{g}_{\bar{\mu}} \oplus \mathbf{n}_{\bar{\mu}}$ and $\mathcal{N}=\mathcal{N}_{0} \oplus \mathcal{N}_{1} \oplus \mathcal{N}_{2}$ the matrix $D_{\mathcal{N}}$ has the structure

$$
D_{\mathcal{N}}=\left(\begin{array}{ccc}
D_{0} & D_{1} & D_{2} \\
0 & 0 & 0
\end{array}\right)
$$

and with respect to the decomposition $\mathbf{g}=\mathbf{g}_{\bar{\mu}} \oplus \mathbf{n}_{\bar{\mu}}$ we have

$$
\operatorname{Ad}_{\bar{\sigma}}^{-1}=\left(\begin{array}{cc}
\left.\operatorname{Ad}_{\bar{\sigma}}^{-1}\right|_{\mathbf{g}_{\bar{\mu}}} & 0 \\
0 & \left.\operatorname{Ad}_{\bar{\sigma}}\right|_{\mathbf{n}_{\bar{\mu}}}
\end{array}\right) .
$$

As before let $\bar{x}$ lie on an RPO with drift-momentum pair $(\bar{\sigma}, \bar{\mu})$. Analogously to the case of relative equilibria, see $(2.9)$, we have

$$
\operatorname{Fix}_{\mathbf{g}_{\bar{\mu}}^{*}}\left(\operatorname{Ad}_{\bar{\sigma}}^{*}\right)=\operatorname{ann}_{\mathbf{g}_{\bar{\mu}}^{*}}\left(\left(\operatorname{Ad}_{\bar{\sigma}}-\mathrm{id}\right) \mathbf{g}_{\bar{\mu}}\right) \simeq \mathbf{g}_{(\bar{\sigma}, \bar{\mu})}^{*},
$$

and a complement of $\mathbf{g}_{(\bar{\sigma}, \bar{\mu})}^{*}$ in $\mathbf{g}_{\bar{\mu}}^{*}$ is given by $\operatorname{ann}_{\mathbf{g}_{\bar{\mu}}^{*}}\left(\mathbf{g}_{(\bar{\sigma}, \bar{\mu})}\right)$. We decompose $\nu \in \mathbf{g}_{\bar{\mu}}^{*}$ as $\nu=\chi+\zeta$ where $\chi \in \mathbf{g}_{(\bar{\sigma}, \bar{\mu})}^{*}$ and $\zeta \in \operatorname{ann}_{\mathbf{g}_{\bar{\mu}}^{*}}\left(\mathbf{g}_{(\bar{\sigma}, \bar{\mu})}\right)$. Let id $\mathcal{X}_{\mathcal{X}}$ denotes the identity on the space $\mathcal{X}$. The following transversality condition for RPOs is a direct extension of Definition 2.7 to RPOs:

Definition 2.20 A relative periodic orbit of a Hamiltonian system (2.1) through $\bar{x} \simeq(0,0, \bar{E}) \in$ $\mathcal{N}$ is called transversal if it is not a relative equilibrium and if

$$
\mathrm{D}_{(\chi, w, E)}\left(\Pi_{\mathcal{N}_{1}}(0,0, \bar{E})-\mathrm{id}_{\mathcal{N}_{1}}\right)
$$

where $\chi \in \mathbf{g}_{(\bar{\sigma}, \bar{\mu})}^{*}$, has full rank.

Note that an RPO through $\bar{x} \simeq(0,0, \bar{E}) \in \mathcal{N}$ is called non-degenerate in [20,24] if $\mathrm{D}_{w} \Pi_{\mathcal{N}_{1}}(0,0, \bar{E})=\mathrm{D}_{x} \Pi^{\bar{E}, \bar{\mu}}(\bar{x})$ does not have 1 as an eigenvalue.

Remark 2.21 If the RPO through $\bar{x}$ has a regular momentum $\bar{\mu}=\mathbf{J}(\bar{x})$ at $\bar{x}$ and $\bar{\sigma} \in \Gamma_{\bar{\mu}}^{\mathrm{id}}$, so that $\alpha=$ id in the decomposition of Lemma 2.14, then $\mathbf{g}_{(\bar{\sigma}, \bar{\mu})}=\mathbf{g}_{\bar{\mu}}$, and $\chi=\nu$ in Definition 2.20. Moreover, $\mathbf{g}_{\mu}$ has constant dimension for $\mu \approx \bar{\mu}$ and, hence, also $\mathcal{S}^{E, \bar{\mu}+\nu}$ has locally constant dimension for $\nu \in \mathbf{g}_{\bar{\mu}}^{*}, \nu \approx 0$. Therefore, we can choose $\mathcal{S}^{E, \bar{\mu}+\nu}$ to depend smoothly on $E$ and $\nu$ and can parametrize it by $w \in \mathcal{N}_{1}$. In this case the RPO is transversal if

$$
\left.\mathrm{D}_{(\nu, w, E)}\left(\Pi^{E, \bar{\mu}+\nu}(x)-\operatorname{id}_{\mathcal{S}_{\bar{E}, \bar{\mu}+\nu}}\right)\right|_{(E=\bar{E}, x=\bar{x}, \nu=0)}
$$

has full rank. This situation is analogous to the case of relative equilibria, which was discussed in Remark 2.8.

Example 2.22 In the case of the symmetry group $\Gamma=\mathrm{SO}(2)$, every momentum $\mu \in \operatorname{so}(2)^{*}$ is regular (see Examples 2.1, 2.2, 2.5), and so $(2.21)$ can be applied to check whether an RPO is transversal.

The following theorem is an extension of the persistence result [20, Theorem 4.2] for nondegenerate RPOs to transversal RPOs:

Theorem 2.23 Let $\Gamma$ be compact. Let $\bar{x}$ lie on a transversal $R P O \overline{\mathcal{P}}$ of $(2.1)$ with relative period $\bar{\tau}$ and regular drift-momentum pair $(\bar{\sigma}, \bar{\mu}) \in\left(\Gamma \times \mathbf{g}^{*}\right)^{c}$. Let $r=r_{(\bar{\sigma}, \bar{\mu})}$, decompose $\bar{\sigma}=\alpha \exp (-\bar{\tau} \bar{\xi})$ as before, and let $\bar{E}=H(\bar{x})$ be the energy of the RPO. Then 
a) there is an $(r+1)$-dimensional family of $R P O s \mathcal{P}(s)$ such that there is a smooth function $x(s), s \in \mathbb{R}^{r+1}$, with $x(0)=\bar{x}$ and $x(s) \in \mathcal{P}(s)$, and at $x(s)$ the $R P O \mathcal{P}(s)$ has

$$
\begin{aligned}
& \text { energy } E(s) \text { close to } E(0)=\bar{E} \text {, } \\
& \text { momentum } \bar{\mu}+\chi(s), \quad \chi(s) \in \mathbf{g}_{(\bar{\sigma}, \bar{\mu})}^{*}, \quad \text { where } \chi(0)=0 \text {, } \\
& \text { relative period } \tau(s) \text { close to } \tau(0)=\bar{\tau} \text {, } \\
& \text { drift symmetry } \sigma(s) \in \Gamma_{(\bar{\sigma}, \bar{\mu})} \text { close to } \sigma(0)=\bar{\sigma} \text {, and } \\
& \text { drift velocity } \xi(s) \in \mathbf{g}_{(\bar{\sigma}, \bar{\mu})} \text { close to } \xi(0)=\bar{\xi} \text {, }
\end{aligned}
$$

where $\sigma(s)=\alpha \exp (-\tau(s) \xi(s)), \operatorname{Ad}_{\alpha} \xi(s)=\xi(s)$.

b) If the $R P O \overline{\mathcal{P}}$ is non-degenerate then we can choose $s=(E, \chi)$.

Note that by the above theorem transversal RPOs of compact group actions persist to nearby energy-level sets and to those nearby momenta which are fixed by the drift symmetry $\bar{\sigma}$ of the $\mathrm{RPO}$ at $\bar{x}$ and lie in a section transverse to the momentum group orbit $\Gamma \bar{\mu}$ (the last condition is only needed in order to guarantee that the RPOs parametrized by $s$ are not symmetry related). During the continuation the momentum $\mu(s)$, the drift symmetry $\sigma(s)$ and the drift velocity $\xi(s)$ of the RPOs $\mathcal{P}(s)$ vary, but the drift symmetry $\alpha$ of the RPOs in the co-moving frame is fixed.

Proof of Theorem 2.23. Since $\operatorname{Ad}_{\bar{\sigma}}$ does not have Jordan blocks, we can conclude from Proposition 2.19 that the RPO is still transversal when considered as an RPO for the symmetry group $\tilde{\Gamma}=\Gamma_{\bar{\sigma}}=Z(\bar{\sigma})$. By Remark 2.17 the isotropy algebra $\mathbf{g}_{(\bar{\sigma}, \bar{\mu})} \subseteq \mathbf{g}_{(\alpha, \bar{\mu})}$ is abelian. This implies, by Lemma $2.18 \mathrm{~b}$ ) applied to the symmetry group $\tilde{\Gamma}$, that $\Pi_{\tilde{\mathcal{N}}_{0}}(\tilde{\nu}, \tilde{w}, E) \equiv \tilde{\nu}$. Here $\tilde{\mathcal{N}}_{0} \simeq \mathbf{g}_{(\bar{\sigma}, \bar{\mu})}^{*},(\tilde{\nu}, \tilde{w}, E) \in \tilde{\mathcal{N}}=\tilde{\mathcal{N}}_{0} \oplus \tilde{\mathcal{N}}_{1} \oplus \tilde{\mathcal{N}}_{2}$ and $\tilde{\mathcal{N}}$ is the slice from Lemma 2.18 for the symmetry group $\tilde{\Gamma}$. Due to Definition 2.20 of transversality we can solve $\Pi_{\tilde{\mathcal{N}}_{1}}(\tilde{\nu}, \tilde{w}, E)=\tilde{w}$ for $(\tilde{\nu}, \tilde{w}, E)(s), s \in \mathbb{R}^{r+1}$, by the implicit function theorem with $\tilde{\nu}(s)=\chi(s) \in \mathbf{g}_{(\bar{\sigma}, \bar{\mu})}^{*}$. This gives an $(r+1)$-dimensional family of RPOs of $(2.1)$ through $x(s) \simeq(\tilde{\nu}, \tilde{w}, E)(s) \in \tilde{\mathcal{N}}$ with relative period $\tau(s)$, drift symmetry $\sigma(s)$ and momentum $\mu(s)=\mathbf{J}(x(s))=\bar{\mu}+\chi(s)$ such that $x(0)=\bar{x}$, $\tau(0)=\bar{\tau}, \sigma(s)=\bar{\sigma}, \chi(0)=0$. By Lemma $2.18 \mathrm{~b}$ ) we have $\sigma(s) \in \tilde{\Gamma}_{\bar{\mu}}=\Gamma_{(\bar{\sigma}, \bar{\mu})}$, and, therefore, we can decompose $\sigma(s)=\alpha \exp (-\tau(s) \xi(s))$ with $\xi(s) \in \mathbf{g}_{(\bar{\sigma}, \bar{\mu})}$ as required.

\subsection{Numerical continuation of transversal RPOs}

In this section we show that the numerical methods presented in [24] for non-degenerate RPOs with regular drift-momentum pair also converge for transversal RPOs of compact symmetry group actions. As in the case of relative equilibria (2.10), we employ the widely used method of adding unfolding parameters to overcome the degeneracies caused by conserved quantities (see e.g. $[6,14]$ and references therein).

Note that by Remark 2.17 the drift symmetry $\bar{\sigma}$ of an RPO is generically regular, and we assume this in the following theorem.

Theorem 2.24 Let $\Gamma$ be compact, let $\bar{x}$ lie on a transversal $R P O \overline{\mathcal{P}}$ with relative period $\bar{\tau}$, regular drift symmetry $\bar{\sigma} \in \Gamma$ and momentum $\bar{\mu}$, and decompose $\bar{\sigma}=\alpha \exp (-\bar{\tau} \bar{\xi})$ as in Lemma 2.14. We set $r=r_{(\bar{\sigma}, \bar{\mu})}$ and denote an orthonormal basis of $\mathbf{g}_{(\bar{\sigma}, \bar{\mu})}$ by $e_{\xi}^{1}, \ldots, e_{\xi}^{r}$. For $\xi \in \mathbf{g}_{(\bar{\sigma}, \bar{\mu})}$ let $\xi=\sum_{i=1}^{r} \xi_{i} e_{\xi}^{i}$ and identify $\xi \simeq\left(\xi_{1}, \ldots, \xi_{r}\right) \in \mathbb{R}^{r}$. As before, let $\mathbf{J}_{i}=\mathbf{J}_{e_{\xi}^{i}}, i=1, \ldots, r$, and define

$$
\dot{x}=f_{H}(x)+\lambda_{E} \nabla H(x)+\sum_{i=1}^{r} \lambda_{\mu, i} \nabla \mathbf{J}_{i}(x)-\sum_{i=1}^{r} \xi_{i} e_{\xi}^{i} .
$$


Denote the flow of (2.22) by $\Phi^{t}\left(x ; \xi, \lambda_{E}, \lambda_{\mu}\right)$. Then the derivative $\mathrm{DF}(y)$ of

$$
F\left(x, T, \xi, \lambda_{E}, \lambda_{\mu}\right)=\alpha \Phi_{\frac{T}{\ell}}\left(x ; \xi, \lambda_{E}, \lambda_{\mu}\right)-x=0,
$$

where

$$
F: \mathcal{X} \times \mathbb{R}^{2+2 r} \rightarrow \mathcal{X}
$$

has full rank at any solution $y=\left(x, T, \xi, \lambda_{E}, \lambda_{\mu}\right)$ of $F=0$ close to $\bar{y}=(\bar{x}, \bar{T}, \bar{\xi}, 0,0)$, where $\bar{T}=\ell \bar{\tau}$. Moreover, any such solution satisfies $\lambda_{E}=0, \lambda_{\mu}=0$, and, hence, determines an $R P O$ of (2.1).

Proof. As in the proof of Theorem 2.23 we replace $\Gamma$ by $\Gamma_{\bar{\sigma}}=Z(\bar{\sigma})$. Since $\mathbf{g}_{\bar{\sigma}}=\mathbf{g}_{(\bar{\sigma}, \bar{\mu})}$, we look for RPOs with drift velocity $\xi \in \mathbf{g}_{\bar{\sigma}}$. We have

$$
\begin{aligned}
\mathrm{D} F(\bar{x}, \bar{T}, \bar{\xi}, 0,0) & =\left[\mathrm{D}_{x} F, \mathrm{D}_{T} F, \mathrm{D}_{\xi} F, \mathrm{D}_{\lambda_{E}} F, \mathrm{D}_{\lambda_{\mu}} F\right] \\
& =\left(\mathrm{D}_{x} \bar{\sigma} \Phi_{\bar{\tau}}(\bar{x})-\mathrm{id}, f_{H}(\bar{x}),-e_{\xi}^{1} \bar{x}, \ldots,-e_{\xi}^{r} \bar{x}, \mathrm{D}_{\lambda_{E}} F, \mathrm{D}_{\lambda_{\mu}} F\right) .
\end{aligned}
$$

The $(r+1, r+1)$-matrix $B$ with

$$
\begin{aligned}
& B_{i j} \quad=\operatorname{DJ}_{i}(\bar{x}) \mathrm{D}_{\lambda_{\mu, j}} F(\bar{y})=\left.\mathrm{DJ}_{i}(\bar{x}) \mathrm{D}_{\lambda_{\mu, j}} \alpha \Phi^{\bar{\tau}}\left(\bar{x} ; \bar{\xi}, 0, \lambda_{\mu}\right)\right|_{\lambda_{\mu}=0}, \\
& i, j=1, \ldots, r \\
& B_{i, r+1}=\mathrm{DJ}_{i}(\bar{x}) \mathrm{D}_{\lambda_{E}} F(\bar{y})=\left.\mathrm{DJ}_{i}(\bar{x}) \mathrm{D}_{\lambda_{E}} \alpha \Phi^{\bar{\tau}}\left(\bar{x} ; \bar{\xi}, \lambda_{E}, 0\right)\right|_{\lambda_{E}=0}, \\
& i=1, \ldots, r \\
& B_{r+1, i}=\mathrm{D} H(\bar{x}) \mathrm{D}_{\lambda_{\mu, i}} F(\bar{y})=\left.\operatorname{DH} H(\bar{x}) \mathrm{D}_{\lambda_{\mu, i}} \alpha \Phi^{\bar{\tau}}\left(\bar{x} ; \bar{\xi}, 0, \lambda_{\mu}\right)\right|_{\lambda_{\mu}=0}, \\
& i=1, \ldots, r \\
& B_{r+1, r+1}=\mathrm{D} H(\bar{x}) \mathrm{D}_{\lambda_{E}} F(\bar{y})=\left.\operatorname{DH}(\bar{x}) \mathrm{D}_{\lambda_{E}} \alpha \Phi^{\bar{\tau}}\left(\bar{x} ; \bar{\xi}, \lambda_{E}, 0\right)\right|_{\lambda_{E}=0}
\end{aligned}
$$

has full rank. This was shown in [24]. We sketch the proof: Since $(\bar{\sigma}, \bar{\mu})$ is regular the isotropy algebra $\mathbf{g}_{(\bar{\sigma}, \bar{\mu})}$ is abelian by Remark 2.17. Consequently, $\Phi^{t}(\cdot ; \xi)=\exp \left(-t \sum_{i=1}^{r} \xi_{i} e_{\xi}^{i}\right) \Phi^{t}(\cdot)$ conserves the momenta $\mathbf{J}_{j}, j=1, \ldots, r$, and as shown in [24], we have

$$
\begin{aligned}
\mathrm{D} H(\bar{x}) \alpha \mathrm{D}_{\lambda_{E}} \Phi^{\bar{\tau}}(\bar{x} ; \bar{\xi}) & =\int_{0}^{\bar{\tau}}\left\|\mathrm{D} H\left(\Phi^{s}(\bar{x} ; \bar{\xi})\right)\right\|^{2} \mathrm{~d} s \\
\operatorname{DJ}_{i}(\bar{x}) \alpha \mathrm{D}_{\lambda_{\mu_{j}}} \Phi^{\bar{\tau}}(\bar{x} ; \bar{\xi}) & =\int_{0}^{\bar{\tau}}\left\langle\nabla \mathbf{J}_{i}\left(\Phi^{s}(\bar{x} ; \bar{\xi})\right), \nabla \mathbf{J}_{j}\left(\Phi^{s}(\bar{x} ; \bar{\xi})\right)\right\rangle \mathrm{d} s \\
\left.\operatorname{DJ}_{i}(\bar{x}) \alpha \mathrm{D}_{\lambda_{E}} \Phi^{\bar{\tau}}(\bar{x} ; \bar{\xi})\right) & =\int_{0}^{\bar{\tau}}\left\langle\mathrm{D} H\left(\Phi^{s}(\bar{x} ; \bar{\xi})\right), \mathrm{DJ}_{i}\left(\Phi^{s}(\bar{x} ; \bar{\xi})\right\rangle \mathrm{d} s\right. \\
\operatorname{DH}(\bar{x}) \alpha \mathrm{D}_{\lambda_{\mu_{i}}} \Phi^{\bar{\tau}}(\bar{x} ; \bar{\xi}) & =\int_{0}^{\bar{\tau}}\left\langle\mathrm{D} H\left(\Phi^{s}(\bar{x} ; \bar{\xi})\right), \mathrm{DJ}_{i}\left(\Phi^{s}(\bar{x} ; \bar{\xi})\right)\right\rangle \mathrm{d} s .
\end{aligned}
$$

Hence, any $c=\left(c_{E}, c_{\mu, 1}, \ldots, c_{\mu, r}\right)$ with $c^{T} B c=0$ satisfies $c_{E} \mathrm{D} H(\bar{x})+\sum_{i=1}^{r} c_{\mu, i} \mathrm{D} \mathbf{J}_{i}(\bar{x})=0$, which contradicts the assumption that $\bar{x}$ does not lie on a relative equilibrium. Therefore $B$ has full rank and is positive definite.

From Proposition 2.19, the assumption of a regular drift symmetry and the above we see that $\operatorname{DF}(\bar{x}, \bar{T}, \bar{\xi}, 0,0)$ has full rank if and only if the matrix $\mathrm{P}_{\mathcal{N}_{1}} \mathrm{D}_{x} F$ has image $\mathcal{N}_{1}$. Here, $\mathrm{P}_{\mathcal{N}_{1}}$ is a projection onto $\mathcal{N}_{1}$ with kernel $\mathbf{g} \bar{x} \oplus \operatorname{span}\left(f_{H}(\bar{x})\right) \oplus \mathcal{N}_{0} \oplus \mathcal{N}_{2}$. Note that

$$
\mathrm{P}_{\mathcal{N}_{1}} \mathrm{D}_{x} F=\left[M_{10}, M_{\mathcal{N}_{1}}-\mathrm{id}_{\mathcal{N}_{1}}, M_{12}\right]=\left[\mathrm{D}_{\nu} \Pi_{\mathcal{N}_{1}}(0), \mathrm{D}_{w} \Pi_{\mathcal{N}_{1}}(0)-\mathrm{id}_{\mathcal{N}_{1}}, \mathrm{D}_{E} \Pi_{\mathcal{N}_{1}}(0)\right],
$$


where we used the notation of Proposition 2.19. Therefore, by Definition 2.20 of transversality, the matrix $\operatorname{DF}(\bar{x}, \bar{T}, \bar{\xi}, 0,0)$ has full rank and $F=0$ has a $(2+2 r)$-dimensional family of solutions.

By Theorem 2.23 there is an $(r+1)$-dimensional manifold $\mathcal{P}(s)$ of RPOs of $(2.1)$ near $\bar{x}$ and $x(s) \in \mathcal{P}(s)$ has drift symmetry $\sigma(s) \in \Gamma_{(\bar{\sigma}, \bar{\mu})}$ and a drift velocity $\xi(s) \in \mathbf{g}_{(\bar{\sigma}, \bar{\mu})}$, which commutes with $\alpha$. Therefore, $y=(x(s), T(s)=\ell \tau(s), \xi(s), 0,0)$, is a solution of $F=0$. Moreover, since, by Remark 2.17, the group $\Gamma_{(\bar{\sigma}, \bar{\mu})}^{\mathrm{id}}$ is abelian, for every $\gamma \in \Gamma_{(\bar{\sigma}, \bar{\mu})}$ and $t \in \mathbb{R}$, the point $y=\left(\gamma \Phi^{t}(x(s)), T(s), \xi(s), 0,0\right)$ is a solution of $F=0$. This gives an $(2 r+2)$-dimensional manifold of solutions of $F=0$. Hence, the $(2 r+2)$-dimensional solution manifold of $F=0$ consists of RPOs of (2.1) near $\bar{y}$ and satisfies $\lambda_{E}=\lambda_{\mu}=0$.

Under the assumptions of Theorem 2.24, the under-determined system (2.23) is amenable to standard numerical methods; for example, the Gauss-Newton method applied to (2.23) converges for initial values $y=\left(x, T, \xi, \lambda_{E}, \lambda_{\mu}\right)$ close to $\bar{y}$.

Remark 2.25 In Theorem 2.23 we only assume that the RPO $\overline{\mathcal{P}}$ has a regular drift-momentum pair $(\bar{\sigma}, \bar{\mu})$ and not necessarily a regular drift symmetry $\bar{\sigma}$ so that in general $\mathbf{g}_{\bar{\sigma}} \neq \mathbf{g}_{(\bar{\sigma}, \bar{\mu})}$. In this case let $e_{\xi}^{1}, \ldots, e_{\xi}^{q}$ denote an orthonormal basis of $\mathbf{g}_{\bar{\sigma}} \operatorname{such}$ that $\operatorname{span}\left(e_{\xi}^{r+1}, \ldots, e_{\xi}^{q}\right)=\mathbf{n}_{\bar{\mu}} \cap \mathbf{g}_{\bar{\sigma}}$ and $\operatorname{span}\left(e_{\xi}^{1}, \ldots, e_{\xi}^{r}\right)=\mathbf{g}_{(\bar{\sigma}, \bar{\mu})}$, Let, as before, $\mathbf{J}_{e_{\xi}^{i}}=\mathbf{J}_{i}$ and for $\mu \in \mathbf{g}^{*}$ let $\mu_{i}=\mu\left(e_{\xi}^{i}\right), i=1, \ldots, q$. Because of Remark 2.17 we generically have $q=r$, but in the case that $q>r$ at the RPO $\overline{\mathcal{P}}$ the derivative $\mathrm{D} F(\bar{y})$ of (2.23) does not have full rank, and convergence of numerical methods applied directly to (2.23) is expected to be slow. In a manner similar to the case of relative equilibria, see Remark 2.11, it is then advantageous to solve the system

$$
F\left(x, T, \xi, \lambda_{E}, \lambda_{\mu}\right)=\left(\begin{array}{l}
\alpha \Phi_{\frac{T}{\ell}}\left(x ; \xi, \lambda_{E}, \lambda_{\mu}\right)-x \\
\mathbf{J}_{r+1}(x)-\bar{\mu}_{r+1} \\
\vdots \\
\mathbf{J}_{q}(x)-\bar{\mu}_{q}
\end{array}\right)=0,
$$

where

$$
F: \mathcal{X} \times \mathbb{R}^{2+q+r} \rightarrow \mathcal{X} \times \mathbb{R}^{q-r}
$$

and $\Phi^{t}\left(x ; \xi, \lambda_{E}, \lambda_{\mu}\right)$ is now the flow of

$$
\dot{x}=f_{H}(x)+\lambda_{E} \nabla H(x)+\sum_{i=1}^{r} \lambda_{\mu, i} \nabla \mathbf{J}_{i}(x)-\sum_{i=1}^{q} \xi_{i} e_{\xi}^{i} .
$$

Note that $\bar{\mu}_{j}=0$ for $j=r+1, \ldots, q$ since $\bar{\mu} \in \mathbf{g}_{(\bar{\sigma}, \bar{\mu})}^{*}$. Because of Proposition 2.19

$$
\begin{aligned}
\mathrm{D} F(\bar{x}, \bar{T}, \bar{\xi}, 0,0) & =\left[\mathrm{D}_{x} F, \mathrm{D}_{T} F, \mathrm{D}_{\xi} F, \mathrm{D}_{\lambda_{E}} F, \mathrm{D}_{\lambda_{\mu}} F\right] \\
& =\left(\begin{array}{lllllll}
\mathrm{D}_{x} \bar{\sigma} \Phi_{\bar{\tau}}(\bar{x})-\mathrm{id} & f_{H}(\bar{x}) & e_{\xi}^{1} \bar{x} & \ldots & e_{\xi}^{q} \bar{x} & \mathrm{D}_{\lambda_{E}} F & \mathrm{D}_{\lambda_{\mu}} F \\
\mathrm{D} \mathbf{J}_{r+1}(\bar{x}) & 0 & 0 & 0 & 0 & 0 & 0 \\
\vdots & 0 & 0 & 0 & 0 & 0 & 0 \\
\mathrm{DJ}_{q}(\bar{x}) & 0 & 0 & 0 & 0 & 0 & 0
\end{array}\right)
\end{aligned}
$$

has full rank and so the solution manifold of $(2.24)$ is $(2 r+2)$-dimensional. As in the proof of Theorem 2.24, for $\gamma$ in the abelian group $\Gamma_{(\bar{\sigma}, \bar{\mu})}^{\mathrm{id}}$ and $t \in \mathbb{R}$, the points $\gamma \Phi^{t}(x(s))$, with $x(s)$ from Theorem 2.23, lie on RPOs $\mathcal{P}(s)$ and have momentum $\mu(s)=\mathbf{J}\left(\gamma \Phi^{t}(x(s))\right)$ satisfying $\mu(s)-\bar{\mu} \in \mathbf{g}_{(\bar{\sigma}, \bar{\mu})}^{*}$, and, thus, $\mu_{j}(s)=\bar{\mu}_{j}, j=r+1, \ldots, q$. Therefore, the set $\{y=$ $\left.\left(\gamma \Phi^{t}(x(s)), T(s), \xi(s), 0,0\right), \gamma \in \Gamma_{(\bar{\sigma}, \bar{\mu})}^{\mathrm{id}}, t \in \mathbb{R}\right\}$ defines a $(2 r+2)$-dimensional solution manifold of (2.24) too. From this $\lambda_{E}=0, \lambda_{\mu}=0$ follows. 
Remark 2.26 In order to continue RPOs in numerically delicate situations, that is, when the single shooting method is ill-conditioned, we use multiple shooting rather than single shooting, cf. [24] and references therein: we compute $k$ points on the RPO in the co-moving frame by solving the under-determined equation

$$
F\left(x_{1}, \ldots, x_{k}, T, \xi, \lambda_{E}, \lambda_{\mu}\right)=0, \quad F: \mathcal{X}^{k} \times \mathbb{R}^{2+2 r} \rightarrow \mathcal{X}^{k} .
$$

Here, $x_{j} \in \mathcal{X}, j=1, \ldots, k, T, \lambda_{E} \in \mathbb{R}, \lambda_{\mu}, \xi \in \mathbb{R}^{r}$. Moreover, $0=s_{1}<\ldots<s_{k+1}=1$ is a partition of the unit interval, $\Delta s_{i}=s_{i+1}-s_{i}$ for $i=1, \ldots k$, and

$$
F_{i}\left(x_{1}, \ldots, x_{k}, T, \xi, \lambda_{E}, \lambda_{\mu}\right)= \begin{cases}\Phi^{\frac{\Delta s_{i} T}{\ell}}\left(x_{i} ; \xi, \lambda_{E}, \lambda_{\mu}\right)-x_{i+1} & \text { for } i=1, \ldots, k-1, \\ \alpha \Phi^{\frac{\Delta s_{k} T}{\ell}}\left(x_{k} ; \xi, \lambda_{E}, \lambda_{\mu}\right)-x_{1} & \text { for } i=k .\end{cases}
$$

It is well-known, see, e.g., [24] and references therein, that the derivative DF of (2.25) has full rank in an RPO if and only if the corresponding derivative of the single shooting equations (2.23) has full rank. Therefore, Theorem 2.24 can readily be applied to the multiple shooting context.

Remark 2.27 The relation

$$
\mathrm{D}_{x} F(\bar{x}, \ell \bar{\tau}, \bar{\xi}, 0,0)=\mathrm{D}_{x} \bar{\sigma} \Phi^{\bar{\tau}}(\bar{x})-\mathrm{id}
$$

between the derivative of the Poincaré-map on one hand, and the shooting equations (2.23) on the other hand plays an important role in the computation of bifurcations, see Section 3 . In the multiple-shooting case the matrix $\mathrm{D}_{x} \bar{\sigma} \Phi^{\bar{\tau}}(\bar{x})$ can be obtained from the linearization of $(2.25)$, for example see [24].

Remark 2.28 Muñoz-Almaraz et al [14] call a periodic orbit of (2.1) with period $\bar{T}$ through $\bar{x}$ normal if, in our notation

$$
\operatorname{range}\left(\mathrm{D}_{x} \Phi^{\bar{T}}(\bar{x})-\mathrm{id}\right)+\operatorname{span}\left(f_{H}(\bar{x})\right)=\operatorname{kerD} \mathbf{J}(\bar{x}) \cap \operatorname{kerD} H(\bar{x}),
$$

see [14, Definition 4]. By projecting both sides of $(2.27)$ onto $\mathcal{N}_{1} \subseteq \operatorname{ker} \mathrm{DJ}(\bar{x}) \cap \operatorname{ker} \mathrm{D} H(\bar{x})$ we see that a normal periodic orbit is transversal. In the case of trivial symmetry $\Gamma=\{\operatorname{id}\}$, a periodic orbit of (2.1) is normal if and only if it is transversal. For nontrivial symmetry groups a periodic orbit which is non-degenerate (and, hence, transversal) as RPO need not be normal as for such an orbit $\mathbf{g}_{\bar{\mu}} \bar{x}$ lies in $\operatorname{DJ}(\bar{x}) \cap \operatorname{kerD} H(\bar{x})$, but might not lie in the image of $\left(\mathrm{D}_{x} \Phi^{\bar{T}}(\bar{x})-\mathrm{id}\right)$. Galan et al. $[6,14]$ prove continuation results for normal periodic orbits and present equations which are satisfied by normal periodic orbits and amenable to numerical continuation methods. These equations are similar to our equations (2.23) in the case of trivial drift symmetry $\bar{\sigma}$, see [14, Theorem 13], but contain additional equations, which ensure that the solution is transverse to the periodic orbit and the group orbit. However they continue periodic orbits, not RPOs. Moreover, they study persistence of normal periodic orbits when external parameters are varied, see $[14$, Theorems 7,14$]$, whereas we restrict attention to continuation in the conserved quantities, energy and momentum, of the system.

\subsection{Numerical path-following of RPOs}

As before let $\bar{x}$ lie on an RPO with drift-momentum pair $(\bar{\sigma}, \bar{\mu})$, let $e_{\xi}^{1}, \ldots, e_{\xi}^{r}$ be an orthonormal basis of $\mathbf{g}_{(\bar{\sigma}, \bar{\mu})}$ and for $\mu \in \mathbf{g}^{*}$ let $\mu_{i}=\mu\left(e_{\xi}^{i}\right), \mathbf{J}_{i}=\mathbf{J}_{e_{\xi}^{i}}, i=1, \ldots, g, g=\operatorname{dim} \Gamma$.

Let us fix the momentum value $\bar{\mu}$ and continue in energy, i.e., solve the equation

$$
F^{\bar{\mu}}\left(x, T, \xi, \lambda_{E}, \lambda_{\mu}\right):=\left(\begin{array}{c}
F\left(x, T, \xi, \lambda_{E}, \lambda_{\mu}\right) \\
\mathbf{J}_{1}(x)-\bar{\mu}_{1} \\
\vdots \\
\mathbf{J}_{r}(x)-\bar{\mu}_{r}
\end{array}\right)=0 .
$$


The $x$-component of the solution $y=(x, T, \xi, 0,0)$ of $(2.28)$ then lies in $\mathcal{X}^{\bar{\mu}}=\mathbf{J}^{-1}(\bar{\mu})$. This can be seen as in Section 2.1.3: Identifying $\mathbf{g}$ with $\mathbf{g}^{*}$ by a $\Gamma$-invariant inner product we get $\bar{\mu}_{j}=0$, $j=r+1, \ldots, g$. For $\sigma \in \Gamma_{\bar{\sigma}}$ close to $\bar{\sigma}$ we have $\mathbf{g}_{\sigma} \subseteq \mathbf{g}_{\bar{\sigma}}, \mathbf{g}_{\sigma}^{*} \subseteq \mathbf{g}_{\bar{\sigma}}^{*}$. By construction, $\xi \in \mathbf{g}_{\bar{\sigma}}$ at any solution $y=(x, T, \xi, 0,0)$ of (2.28). Since the drift symmetry $\sigma=\alpha \exp (-\tau \xi), \tau=T / \ell$, and momentum $\mu=\mathbf{J}(x)$ of the RPO given by $y=(x, T, \xi, 0,0)$ satisfy $\operatorname{Ad}_{\sigma}^{*} \mu=\mu$ for (see $(2.15)$ ), we therefore conclude from (2.20) that $\mu \in \mathbf{g}_{\bar{\sigma}}^{*}$ and, hence, $\mathbf{J}_{j}(x)=0, j=q+1, \ldots, g$. If $q \neq r$ then $\mu_{j}=\bar{\mu}_{j}, j=r+1, \ldots, q$ is one of the equations of $F$, see (2.24). Hence, the solutions of (2.28) satisfy $\mathbf{J}(x)=\bar{\mu}$.

Alternatively, we fix the energy and $g-1$ out of the first $g$ momentum components to get a one-parameter family of RPOs, without loss of generality the $g-1$ components $\bar{\mu}^{b}=\left(\bar{\mu}_{2}, \ldots, \bar{\mu}_{g}\right)$ by solving

$$
F^{\bar{E}, \bar{\mu}^{\mathrm{b}}}\left(x, \xi, T, \lambda_{E}, \lambda_{\mu}\right):=\left(\begin{array}{c}
F\left(x, \xi, \lambda_{E}, \lambda_{\mu}\right) \\
\mathbf{J}_{2}(x)-\bar{\mu}_{2} \\
\vdots \\
\mathbf{J}_{r}(x)-\bar{\mu}_{r} \\
H(x)-\bar{E}
\end{array}\right)=0 .
$$

As above, we see that the solutions $y=(x, T, \xi, 0,0)$ of $(2.29)$ satisfy

$$
x \in \mathcal{X}^{\bar{E}, \mu^{b}}=\left\{x \in \mathcal{X}, \quad H(x)=\bar{E}, \quad \mathbf{J}_{j}(x)=\bar{\mu}_{j}, j=2, \ldots, g\right\} .
$$

Corollary 2.29 Let $\bar{x}$ lie on transversal RPO with regular drift-momentum pair $(\bar{\sigma}, \bar{\mu})$, relative period $\bar{\tau}$, and energy $\bar{E}$, define the Poincaré-map $\Pi$ as before and choose coordinates as in Lemma 2.18. Decompose $\bar{\sigma}=\alpha \exp (-\bar{\tau} \bar{\xi})$, where $\bar{\xi} \in \mathbf{g}_{(\bar{\sigma}, \bar{\mu})}$, as in Lemma 2.14, and let $\bar{T}=\ell \bar{\tau}$ be the period of the RPO in the co-moving frame $\bar{\xi}$. Then the matrix

$$
\left[\mathrm{D}_{E} \Pi_{\mathcal{N}_{1}}(0,0, \bar{E}), \mathrm{D}_{w} \Pi_{\mathcal{N}_{1}}(0,0, \bar{E})-\mathrm{id}_{\mathcal{N}_{1}}\right]
$$

has full rank if and only if the $(r, r+1)$-matrix $\left.\partial_{s} \chi(s)\right|_{s=0}$ from Theorem 2.23 has full rank. In this case there is a path $x(\epsilon) \in \mathbf{J}^{-1}(\bar{\mu})$ of points on $R P O s \mathcal{P}(\epsilon)$ with energy $E(\epsilon)$, relative period $\tau(\epsilon)$ and drift symmetry $\sigma(\epsilon)=\alpha \exp (-\tau(\epsilon) \xi(\epsilon)), \xi(\epsilon) \in \mathbf{g}_{(\bar{\sigma}, \bar{\mu})}$, such that $x(0)=\bar{x}, \mathcal{P}(0)=\overline{\mathcal{P}}$, $E(0)=\bar{E}, \sigma(0)=\bar{\sigma}, \xi(0)=\bar{\xi}, \tau(0)=\bar{\tau}$. If the RPO $\overline{\mathcal{P}}$ is non-degenerate, we can choose $\epsilon=E$. Moreover, $\mathrm{DF}^{\bar{\mu}}(\bar{x}, \bar{T}, \bar{\xi}, 0,0)$ has full rank and $(x(\epsilon), T(\epsilon), \xi(\epsilon), 0,0)$, where $T(\epsilon)=\ell \tau(\epsilon)$, solves $(2.28)$

If the matrix in (2.30) has full rank we say that the RPO $\overline{\mathcal{P}}$ is transversal with respect to $C(x):=H(x)$. Under this assumption, (2.28) can be solved by standard numerical methods, for example by the Gauss-Newton method, for initial values close to $\bar{y}=(\bar{x}, \bar{T}, \bar{\xi}, 0,0)$.

Corollary 2.30 Let $\bar{x}$ lie on transversal RPO with regular drift-momentum pair $(\bar{\sigma}, \bar{\mu})$, relative period $\bar{\tau}$, and energy $\bar{E}$, define the Poincaré-map $\Pi$ as before and choose coordinates as in Lemma 2.18. Decompose $\bar{\sigma}=\alpha \exp (-\bar{\tau} \bar{\xi})$, where $\bar{\xi} \in \mathbf{g}_{(\bar{\sigma}, \bar{\mu})}$, as in Lemma 2.14, and let $\bar{T}=\ell \bar{\tau}$ be the period of the RPO in the co-moving frame $\bar{\xi}$. Then the matrix

$$
\left[\mathrm{D}_{\chi_{1}} \Pi_{\mathcal{N}_{1}}(0,0, \bar{E}), \mathrm{D}_{w} \Pi_{\mathcal{N}_{1}}(0,0, \bar{E})-\mathrm{id}_{\mathcal{N}_{1}}\right]
$$

has full if and only if the $(r, r+1)$-matrix $\left.\partial_{s}\left(E, \chi_{2}, \ldots, \chi_{r}\right)(s)\right|_{s=0}$ from Theorem 2.23 has full rank. In this case there is a path $x(\epsilon) \in \mathcal{X}^{\bar{E}, \bar{\mu}^{b}}$ of points on $R P O s \mathcal{P}(\epsilon)$ with energy $\bar{E}$, relative period $\tau(\epsilon)$ and drift symmetry $\sigma(\epsilon)=\alpha \exp (-\tau(\epsilon) \xi(\epsilon)), \xi(\epsilon) \in \mathbf{g}_{(\bar{\sigma}, \bar{\mu})}$, such that $x(0)=\bar{x}$, $\mathcal{P}(0)=\overline{\mathcal{P}}, \mu_{1}(0)=\bar{\mu}_{1}, \sigma(0)=\bar{\sigma}, \xi(0)=\bar{\xi}, \tau(0)=\bar{\tau}$. If the $R P O \mathcal{\mathcal { P }}$ is non-degenerate we can choose $\epsilon=\mu_{1}$. Moreover, $\mathrm{D}^{\bar{E}, \bar{\mu}^{b}}(\bar{x}, \bar{T}, \bar{\xi}, 0,0)$ has full rank and $(x(\epsilon), T(\epsilon), \xi(\epsilon), 0,0)$, where $T(\epsilon)=\ell \tau(\epsilon)$, solves $(2.29)$. 
If the matrix in (2.31) has full rank we say that the RPO $\overline{\mathcal{P}}$ is transversal with respect to $C(x):=\mathbf{J}_{1}(x)$ (analogously to the case of relative equilibria, see Section 2.1.3). Under this assumption, (2.29) can be solved by standard numerical methods, for example by the GaussNewton method, for initial values close to $\bar{y}=(\bar{x}, \bar{T}, \bar{\xi}, 0,0)$.

For continuation we use the tangent $t(\bar{y})$ to the solution manifold of $F^{\bar{E}, \bar{\mu}^{b}}=0$ or $F^{\bar{\mu}}=0$ at $\bar{y}$ that lies in the kernel of $\mathrm{D} F^{\bar{E}, \bar{\mu}^{b}}(\bar{y})$ or $\mathrm{D} F^{\bar{\mu}}(\bar{y})$, respectively, and has an $x$-component $t_{x}=t_{x}(\bar{y})$ orthogonal to $\mathbf{g}_{\bar{\sigma}} \bar{x}$ and $f_{H}(\bar{x})$.

Remark 2.31 In the multiple shooting context, let $F^{\bar{\mu}}: \mathcal{X}^{k} \times \mathbb{R}^{2 r+2} \rightarrow \mathcal{X}^{k} \times \mathbb{R}^{r}$ or $F^{\bar{E}, \bar{\mu}^{b}}$ : $\mathcal{X}^{k} \times \mathbb{R}^{2 r+2} \rightarrow \mathcal{X}^{k} \times \mathbb{R}^{r}$, respectively, be given by (2.25) with $r$ conserved quantities fixed as in (2.28) or (2.29). At an RPO which is transversal with respect to $H(x)$ or $\mathbf{J}_{1}(x)$, the kernel of $\mathrm{D}^{\bar{\mu}} F(\bar{y})$ or $\mathrm{D}^{\bar{E}, \bar{\mu}^{b}} F(\bar{y})$, respectively, is $(r+1)$-dimensional by Corollaries 2.29 and 2.30. We write the vectors in this kernel as $t=\left(t_{1}, \ldots, t_{k}, t_{T}, t_{\xi}, t_{\lambda_{E}}, t_{\lambda_{\mu}}\right)$, where $t_{j} \in \mathbb{R}^{2 d}, j=1, \ldots, k$, $t_{\xi}, t_{\lambda_{\mu}} \in \mathbb{R}^{r}, t_{E}, t_{\lambda_{E}} \in \mathbb{R}$. Then the kernel contains the vectors $t^{f}, t^{\xi_{1}}, \ldots, t^{\xi_{r}}$ where

$$
t_{i}^{f}=f\left(x_{i}\right), \quad i=1, \ldots, k, \quad t_{T}^{f}=t_{\lambda_{E}}^{f}=0, \quad t_{\xi}^{f}=t_{\lambda_{\mu}}^{f}=0,
$$

and

$$
t_{i}^{\xi_{j}}=e_{\xi}^{j} x_{i}, \quad i=1, \ldots, k, \quad t_{T}^{\xi_{j}}=t_{\lambda_{E}}^{\xi_{j}}=0, \quad t_{\xi}^{\xi_{j}}=t_{\lambda_{\mu}}^{\xi_{j}}=0, \quad j=1, \ldots, r .
$$

We define the continuation tangent $t=\left(t_{1}, \ldots, t_{k}, t_{T}, t_{\xi}, t_{\lambda_{E}}=0, t_{\lambda_{\mu}}=0\right)$ as the element of the kernel which is orthogonal to $t^{f}$ and $t^{\xi_{1}}, \ldots, t^{\xi_{r}}$, as in [24] in the case of non-degenerate RPOs.

Remark 2.32 In the actual implementation it is more convenient to add the continuation parameter $C=E$ or $C=\mu_{1}$, respectively, to the vector of unknowns $y=\left(x, T, \xi, \lambda_{E}, \lambda_{\mu}, C\right)$ and to add the additional equation $C(x)-C=0$ to $F$. Then the $C$-component $t_{C}$ of the continuation tangent at $\bar{y}$ satisfies $t_{C}=\mathrm{D} C(\bar{x}) t_{x}$ as required, and computing boundary points in $C$ is greatly simplified.

\subsection{Turning points of relative equilibria and RPOs}

In this section we deal with the simplest situation of a critical relative equilibrium or RPO, namely, we consider a transversal relative equilibrium/RPO with regular velocity-momentum pair/drift-momentum pair, which ceases to be non-degenerate.

\subsubsection{Turning points of Hamiltonian relative equilibria}

Let $\bar{x}$ lie on a transversal relative equilibrium with regular velocity-momentum pair $(\bar{\xi}, \bar{\mu})$ and assume that the relative equilibrium is degenerate. Let $r=r_{(\bar{\xi}, \bar{\mu})}$. By Theorem 2.9 the relative equilibrium $\Gamma \bar{x}$ persists to an $r$-dimensional family $\Gamma x(s)$ of relative equilibria nearby with velocity-momentum pairs $(\xi(s), \mu(s)=\bar{\mu}+\chi(s))$ at $x(s), \chi(s) \in \mathbf{g}_{(\bar{\xi}, \bar{\mu})}^{*} \simeq \mathbb{R}^{r}$. Since the relative equilibrium is degenerate the matrix $\mathbb{J}_{\mathcal{N}_{1}} \mathrm{D}_{w}^{2} h(0)$ in (2.7) has an eigenvalue 0 . Therefore, we see from the proof of Theorem 2.9 that the $(r, r)$-matrix $\frac{\partial \chi}{\partial s}(0)$ is singular. The eigenvalue 0 of $\mathbb{J}_{\mathcal{N}_{1}} \mathrm{D}_{w}^{2} h(0)$ is of algebraic multiplicity two since $\mathbb{J}_{\mathcal{N}_{1}} \mathrm{D}_{w}^{2} h(0)$ is infinitesimally symplectic, and, generically, of geometric multiplicity one, see [12]. As discussed in Section 2.1.3, we numerically compute a one-parameter family of relative equilibria by fixing all components of the momentum map except for the first component, $C(x):=\mathbf{J}_{1}(x)$.

Proposition 2.33 Assume that the relative equilibrium through $\bar{x}$ is degenerate, but transversal with respect to the conserved quantity $C(x):=\mathbf{J}_{1}(x)$ of $(2.1)$. Let $x(\epsilon) \in \mathcal{X}^{\bar{\mu}^{b}}$ be the path of relative equilibria from Corollary 2.13. Then generically $\bar{x}$ is a turning point in $c(\epsilon)=C(x(\epsilon))$, 
i.e., $c^{\prime}(0)=0$ and $c^{\prime \prime}(0) \neq 0$, and the pair of eigenvalues of the linearization of the relative equilibria, which collide at 0 at the turning point, lies on the imaginary axis before the turning point is passed, and on the real axis after the turning point, or vice versa.

Proof. As we see from the proof of Theorem 2.9, the relative equilibria through $x(s) \simeq$ $(\tilde{\nu}(s), \tilde{w}(s)) \in \tilde{\mathcal{N}}_{0} \oplus \tilde{\mathcal{N}}_{1}$ correspond to equilibria of the $\tilde{\nu}$-dependent Hamiltonian system $\frac{\mathrm{d} \tilde{w}}{\mathrm{~d} t}=$ $f_{\tilde{\mathcal{N}}_{1}}(\tilde{\nu}, \tilde{w})$ after symmetry reduction by $\tilde{\Gamma}=\Gamma_{\bar{\xi}}$. Here, $\tilde{\nu}=\chi$ and so $\tilde{\nu}_{j}=0, j=2, \ldots, r, \tilde{\nu}_{1}=c$. Define the parameter-dependent Hamiltonian vector-field $f_{\tilde{\mathcal{N}}_{1}}(c, \tilde{w})$ by choosing $\tilde{\nu}$ in this way. By assumption $\mathrm{D}_{\tilde{w}} f_{\tilde{\mathcal{N}}_{1}}(0,0)$ has a one-dimensional kernel and $\left[\mathrm{D}_{c} f_{\tilde{\mathcal{N}}_{1}}(0,0), \mathrm{D}_{\tilde{w}} f_{\tilde{\mathcal{N}}_{1}}(0,0)\right]$ has full rank. Denote the solutions of $f_{\tilde{\mathcal{N}}_{1}}(c, \tilde{w})=0$ corresponding to $x(\epsilon)$ as $(c(\epsilon), \tilde{w}(\epsilon))$ where $\bar{c}=c(0)$. Since $\mathrm{D}_{\tilde{w}} f_{\tilde{\mathcal{N}}_{1}}(\bar{c}, 0) w^{\prime}(0)+\mathrm{D}_{c} f_{\tilde{\mathcal{N}}_{1}}(0,0) c^{\prime}(0)=0$ we infer from the above that $\mathrm{D}_{\tilde{w}} f_{\tilde{\mathcal{N}}_{1}}(0,0) w^{\prime}(0)=0$, $\mathrm{D}_{c} f_{\tilde{\mathcal{N}}_{1}}(\bar{c}, 0) \neq 0$, and so $c^{\prime}(0)=0$. Moreover, applying well-known results on turning points of Hamiltonian systems on the Hamiltonian vector-field $f_{\tilde{\mathcal{N}}_{1}}(c, \tilde{w})$, we see that generically $c^{\prime \prime}(0) \neq 0$ and that the pair of eigenvalues of $\mathrm{D}_{\tilde{w}} f_{\tilde{\mathcal{N}}_{1}}(c(\epsilon), \tilde{w}(\epsilon))$, which collide at 0 at $\epsilon=0$, is on the imaginary axis for $\epsilon<0(\epsilon>0)$ and on the real axis for $\epsilon>0(\epsilon<0)$.

\subsubsection{Turning points of RPOs}

Let $\bar{x}$ lie on a transversal RPO with relative period $\bar{\tau}$, regular drift-momentum pair $(\bar{\sigma}, \bar{\mu})$ and energy $\bar{E}$, and assume that the RPO is degenerate. Let $r=r_{(\bar{\sigma}, \bar{\mu})}$. By Theorem 2.23 it persists to an $(r+1)$-dimensional family $\mathcal{P}(s)$ of RPOs with drift-momentum pairs $(\sigma(s), \mu(s)=\bar{\mu}+\chi(s))$ and energy $E(s)$, where $\chi \in \mathbf{g}_{(\bar{\sigma}, \bar{\mu})}^{*} \simeq \mathbb{R}^{r}$ at $x(s) \in \mathcal{P}(s)$. Since the RPO is degenerate the matrix $\mathrm{D}_{w} \Pi_{\mathcal{N}_{1}}(0,0, \bar{E})=\mathrm{D}_{x} \Pi^{E, \mu}(\bar{x})$ has an eigenvalue 1 . Therefore, we see from the proof of Theorem 2.23 that the $(r+1, r+1)$-matrix $\frac{\partial}{\partial s}(E, \chi)(0)$ is singular.

As discussed in Section 2.4, we numerically compute a one-parameter family of RPOs and continue RPOs with respect to a component of the momentum map or the energy. Then we have:

Proposition 2.34 Assume that the RPO through $\bar{x}$ is degenerate, but transversal with respect to the conserved quantity $C(x):=H(x)$ or $C(x):=\mathbf{J}_{1}(x)$ of $(2.1)$. Denote by $x(\epsilon) \in \mathcal{X}^{\bar{\mu}}$ or $x(\epsilon) \in \mathcal{X}^{E, \bar{\mu}^{b}}$ the path of RPOs from Corollary 2.29 or Corollary 2.30, and let $c(\epsilon)=C(x(\epsilon))$. Then generically $\bar{x}$ is a turning point in $c(\epsilon)=C(x(\epsilon))$, i.e., $c^{\prime}(0)=0$ and $c^{\prime \prime}(0) \neq 0$, and the pair of eigenvalues of the linearization of the RPOs, which collide at 1 at the turning point, lies on the unit circle before the turning point is passed, and on the real axis after the turning point, or vice versa.

Proof. From the proof of Theorem 2.23 we see that the path of RPOs through $x(\epsilon) \simeq$ $(\tilde{\nu}(\epsilon), \tilde{w}(\epsilon), E(\epsilon))$, where $x(0)=\bar{x} \simeq(0,0, \bar{E})$, corresponds to fixed points of the $(\tilde{\nu}, E)$-dependent symplectic map $\Pi_{\widetilde{\mathcal{N}}_{1}}(\tilde{\nu}, \tilde{w}, E)$ after reduction by $\tilde{\Gamma}=\Gamma_{\bar{\sigma}}$. Here $\tilde{\nu}=\chi \in \mathbf{g}_{(\bar{\sigma}, \bar{\mu})}^{*}$, so that in the case $C(x)=H(x)$ we have $\tilde{\nu} \equiv 0, c=E$, and in the case $C(x)=\mathbf{J}_{1}(x)$ we have $\tilde{\nu}_{j} \equiv 0, j=2, \ldots, r, E=\bar{E}, c=\tilde{\nu}_{1}$. With this choice of $\tilde{\nu}$ we obtain a symplectic map $\Pi_{\widetilde{\mathcal{N}}_{1}}(c, \tilde{w})$ which depends on one parameter $c$. At $\bar{c}=C(\bar{x}), \tilde{w}=0$, the derivative $\mathrm{D}_{\tilde{w}} \Pi_{\widetilde{\mathcal{N}}_{1}}(\bar{c}, 0)$, has an eigenvalue 1 with geometric multiplicity one and algebraic multiplicity two such that $\left[\mathrm{D}_{c} \Pi_{\widetilde{\mathcal{N}}_{1}}(\bar{c}, 0), \mathrm{D}_{\tilde{w}} \Pi_{\tilde{\mathcal{N}}_{1}}(\bar{c}, 0)-\mathrm{id}_{\widetilde{\mathcal{N}}_{1}}\right]$ has full rank. Since $\bar{x}$ is degenerate, but transversal, the equation $\left(\mathrm{D}_{\tilde{w}} \Pi_{\tilde{\mathcal{N}}_{1}}(\bar{c}, 0)-\mathrm{id}_{\tilde{\mathcal{N}}_{1}}\right) \tilde{w}^{\prime}(0)+\mathrm{D}_{c} \Pi_{\mathcal{N}_{1}}(\bar{c}, 0) c^{\prime}(0)=0$ implies $\left(\mathrm{D}_{\tilde{w}} \Pi_{\tilde{\mathcal{N}}_{1}}(\bar{c}, 0)-\mathrm{id}\right) \tilde{w}^{\prime}(0)=0$ and $\mathrm{D}_{c} \Pi_{\tilde{\mathcal{N}}_{1}}(\bar{c}, 0) \neq 0$. Hence, $c^{\prime}(0)=0$. Applying the well-known results on turning points of parameter-dependent symplectic maps, see, e.g., [12], on the symplectic map $\Pi_{\widetilde{\mathcal{N}}_{1}}(c, \cdot)$ then completes the proof. 


\subsubsection{Detection and computation of turning points of relative equilibria and RPOs}

Turning points along paths of RPOs continued in the conserved quantity $C(x)$ can be detected by a sign change of

$$
u(y)=\left\langle t_{x}, \nabla C(x)\right\rangle
$$

at two consecutively computed solutions $y^{(0)}=\left(x^{(0)}, T^{(0)}, \xi^{(0)}, 0\right)$ and $y^{(1)}=\left(x^{(1)}, T^{(1)}, \xi^{(1)}, 0\right)$ of (2.28) or (2.29), respectively. Here, $t_{x}$ is the $x$-component of the continuation tangent $t(y)$ at $y=(x, T, \xi, 0)$. Once detected, turning points can be computed by a combination of Hermite interpolation and subdivision of (2.34) along the solution path of (2.28) or (2.29), see, e.g., [23] and references therein.

Analogously, turning points of paths of relative equilibria which are continued in $C(x)=$ $\mathbf{J}_{1}(x)$ are detected by a sign change of (2.34) at two consecutively computed solutions of (2.13) and computed by subdivision of (2.34) along the solution path of (2.13).

\section{$3 \quad$ Hamiltonian relative period doubling bifurcations}

In this section we first present a theorem on relative period doubling bifurcations of RPOs with regular drift-momentum pair (Section 3.1). Then, in Sections 3.2 and 3.3, we show how to detect and compute relative period doubling bifurcations along branches of RPOs. In Section 3.4 we deal with the detection and computation of relative period halving bifurcations during numerical path-following of RPOs.

\subsection{A theorem on relative period doubling bifurcations of RPOs}

Let $\bar{x}$ lie on a non-degenerate RPO $\overline{\mathcal{P}}$ with regular drift-momentum pair $(\bar{\sigma}, \bar{\mu})$ and energy $\bar{E}$. We say that $\bar{x}$ is a relative period doubling bifurcation point if $\mathrm{D}^{\bar{E}, \bar{\mu}}(\bar{x})=\mathrm{D}_{w} \Pi_{\mathcal{N}_{1}}(0,0, \bar{E})$ has an eigenvalue -1 . This eigenvalue has algebraic multiplicity $\geq 2$ since, by Lemma $2.18 \mathrm{~b}$ ), the map $\Pi_{\mathcal{N}_{1}}$ is symplectic. We make the generic assumption that the eigenvalue -1 of $\mathrm{D}_{w} \Pi_{\mathcal{N}_{1}}(0,0, \bar{E})$ has algebraic multiplicity 2 and geometric multiplicity 1.

Let $x(\chi, E), \chi \in \mathbf{g}_{(\bar{\sigma}, \bar{\mu})}^{*}$, be the family of RPOs through $\bar{x}=x(0, \bar{E})$ whose existence was proved in Theorem 2.23. Denote its coordinates on $\mathcal{N}$ as $(\nu(\chi, E), w(\chi, E), E)$. Let $\lambda_{1}(\chi, E)$ and $\lambda_{2}(\chi, E)$ be the eigenvalues of $\mathrm{D}_{w} \Pi_{\mathcal{N}_{1}}(\nu(\chi, E), w(\chi, E), E)$ which collide at the bifurcation: $\lambda_{1}(0, \bar{E})=\lambda_{2}(0, \bar{E})=-1$. Denote the generalized eigenspace of $\mathrm{D}_{w} \Pi_{\mathcal{N}_{1}}(\nu(\chi, E), w(\chi, E), E)$ to the eigenvalues $\lambda_{1}(\chi, E)$ and $\lambda_{2}(\chi, E)$ by $\mathcal{Y}(\chi, E)$. Then $\mathcal{Y}(\chi, E)$ is a symplectic space, the matrix

$$
B(\chi, E):=\left.\mathrm{D}_{w} \Pi_{\mathcal{N}_{1}}(\nu(\chi, E), w(\chi, E), E)\right|_{\mathcal{Y}(\chi, E)}
$$

is a symplectic $(2,2)$-matrix, and $\mathcal{Y}(\chi, E)$ and $B(\chi, E)$ depend smoothly on $(\chi, E)$. If

$$
\psi(\chi, E):=\operatorname{tr}(B(\chi, E))+2=0,
$$

then $B(\chi, E)$ has a double eigenvalue -1 . We assume that

$$
\mathrm{D}_{(\chi, E)} \psi(0, \bar{E}) \neq 0 \text {. }
$$

In [12] it is shown that this condition is generically satisfied in the space $\operatorname{SP}(2)$ of symplectic $(2,2)$-matrices. Under this condition the equation $\psi(\chi, E)=0$ determines a smooth hypersurface of co-dimension 1 in $(\chi, E)$-space. We choose coordinates such that $\mathrm{D}_{\left(\chi_{1}, E\right)} \psi(0, \bar{E}) \neq 0$.

Remark 3.1 At a transverse passing of the hyper-surface $\psi(\chi, E)=0$ in $\operatorname{SP}(2)$ the eigenvalues are either on the unit circle before the collision and on the real axis after, or vice versa. To 
see this, let $\lambda_{1}(\chi, E)=-1+\delta(\chi, E)$ where $\delta(0, \bar{E})=0$. Then $\lambda_{2}(\chi, E)=1 /(-1+\delta(\chi, E))=$ $-1-\delta(\chi, E)-\delta^{2}(\chi, E)+$ h.o.t., and so $\psi(\chi, E)=-\delta^{2}(\chi, E)+$ h.o.t. As $\psi(\chi, E)$ changes sign at the relative period-doubling hyper-surface determined by $(3.1)$, we see that $\delta(E, \chi)$ is real before bifurcation and complex after bifurcation, or vice versa.

As before, define $\widetilde{\mathcal{N}}=\widetilde{\mathcal{N}}_{0} \oplus \widetilde{\mathcal{N}}_{1} \oplus \widetilde{\mathcal{N}}_{2}$ to be the linear section transverse to the RPO through $\bar{x}$ from Lemma 2.18 for the symmetry group $\tilde{\Gamma}=\Gamma_{\bar{\sigma}}$. We denote the eigenvector of $\mathrm{D}_{\tilde{w}} \Pi_{\widetilde{\mathcal{N}}_{1}}(0,0, \bar{E})$, $\tilde{w} \in \widetilde{\mathcal{N}}_{1}$, to the eigenvalue -1 by $\bar{w}$. In the following theorem we make the generic assumption that $\mathrm{D}_{E} \psi(0, \bar{E}) \neq 0$. We deal with the case $\mathrm{D}_{E} \psi(0, \bar{E})=0, \mathrm{D}_{\chi_{1}} \psi(0, \bar{E}) \neq 0$ in Remark 3.3 below.

Theorem 3.2 Let $\bar{x}$ be a relative period doubling bifurcation point with a regular drift-momentum pair $(\bar{\sigma}, \bar{\mu})$, with relative period $\bar{\tau}$ and energy $\bar{E}$. Decompose $\bar{\sigma}$ as in Lemma 2.14. Assume that $\left(\bar{\sigma}^{2}, \bar{\mu}\right)$ is also a regular drift-momentum pair and that $r=r_{(\bar{\sigma}, \bar{\mu})}(\Gamma)=r_{\left(\bar{\sigma}^{2}, \bar{\mu}\right)}(\Gamma)$. Then, generically, a family $\tilde{x}(\epsilon, \chi), \epsilon \geq 0, \chi \in \mathbf{g}_{(\bar{\sigma}, \bar{\mu})}^{*}, \epsilon, \chi \approx 0$, of points on $\operatorname{RPOs} \tilde{\mathcal{P}}(\epsilon, \chi)$ with relative period $\tilde{\tau}(\epsilon, \chi)$, drift symmetry $\tilde{\sigma}(\epsilon, \chi) \in \Gamma_{(\bar{\sigma}, \bar{\mu})}$, momentum $\tilde{\mu}(\epsilon, \chi)=\bar{\mu}+\chi$, and energy $\tilde{E}(\epsilon, \chi)$ bifurcates from $\bar{x}$ such that

$$
\tilde{x}(0,0)=\bar{x}, \quad \tilde{\tau}(0,0)=2 \bar{\tau}, \quad \tilde{\sigma}(0,0)=\bar{\sigma}^{2}, \quad \tilde{\mu}(0)=\bar{\mu}, \quad \tilde{E}(0)=\bar{E} .
$$

Moreover,

$$
\tilde{\sigma}(\epsilon, \chi)=\alpha^{2} \exp (-\tilde{\tau}(\epsilon, \chi) \tilde{\xi}(\epsilon, \chi))
$$

with

$$
\tilde{\xi}(s) \in \mathbf{g}_{(\bar{\sigma}, \bar{\mu})}, \quad \operatorname{Ad}_{\bar{\alpha}} \tilde{\xi}(\epsilon, \chi)=\tilde{\xi}(\epsilon, \chi),
$$

and

$$
\mathrm{D}_{\epsilon} \tilde{x}(0,0)=\bar{w}, \quad \mathrm{D}_{\epsilon} \tilde{\tau}(0,0)=0, \quad \mathrm{D}_{\epsilon} E(0,0)=0, \quad \mathrm{D}_{\epsilon} \tilde{\sigma}(0,0)=0, \quad \mathrm{D}_{\epsilon} \tilde{\xi}(0,0)=0 .
$$

Proof. We reduce by $\tilde{\Gamma}=\Gamma_{\bar{\sigma}}$ only. Since $\Gamma$ is compact the matrices $\operatorname{Ad}_{\bar{\sigma}}$ and $\left.\operatorname{Ad}_{\bar{\sigma}}^{*}\right|_{\mathbf{g}_{\mu}^{*}}$ do not have Jordan blocks. Therefore, we conclude from (2.19) that the RPO through $\bar{x}$ is nondegenerate when considered as an RPO for the symmetry group $\tilde{\Gamma}=\Gamma_{\bar{\sigma}}$. Then, as before, since $\mathbf{g}_{(\bar{\sigma}, \bar{\mu})}$ is abelian by Remark 2.17, we have $\Pi_{\tilde{\mathcal{N}}_{0}}(\tilde{\nu}, \tilde{w}, E) \equiv \tilde{\nu}$, where $\tilde{\nu}=\chi \in \mathbf{g}_{(\bar{\sigma}, \bar{\mu})}^{*} \simeq \mathbb{R}^{r}$. Here, $\tilde{\mathcal{N}}_{0} \simeq \mathrm{g}_{(\bar{\sigma}, \bar{\mu})}^{*},(\tilde{\nu}, \tilde{w}, E) \in \tilde{\mathcal{N}}=\tilde{\mathcal{N}}_{0} \oplus \tilde{\mathcal{N}}_{1} \oplus \tilde{\mathcal{N}}_{2}$. Moreover, if we reduce by $\tilde{\Gamma}$ only, then $\mathrm{D}_{\tilde{w}} \Pi_{\tilde{\mathcal{N}}_{1}}(0,0, \bar{E})$ still has an eigenvalue -1 of multiplicity two and the symplectic map $\Pi_{\widetilde{\mathcal{N}}_{1}}(\tilde{\nu}, \cdot, E)$ undergoes a period-doubling bifurcation at $E=\bar{E}, \tilde{\nu}=0$.

Since $\mathrm{D}_{E} \psi(0, \bar{E}) \neq 0$, on the manifold of fixed points $\tilde{w}(E, \tilde{\nu})$ of $\Pi_{\tilde{\mathcal{N}}_{1}}(\tilde{\nu}, \tilde{w}, E)$ corresponding to RPOs $\mathcal{P}(\chi, E), \chi=\tilde{\nu}$, of (2.1) there is a co-dimension one manifold $\tilde{w}(\chi)$ with parameters $\tilde{\nu}=\chi$ and $E=E(\chi)$ such that $\left.\mathrm{D}_{w} \Pi_{\tilde{\mathcal{N}}_{1}}(\tilde{\nu}, \tilde{w}, E)(\chi)\right)$ has an eigenvalue -1 , where $\chi \in \mathbf{g}_{(\bar{\sigma}, \bar{\mu})}^{*}$. Then, generically, the parameter dependent equation

$$
\Pi_{\widetilde{\mathcal{N}}_{1}}^{2}(\tilde{\nu}, \tilde{w}, E)=\tilde{w}
$$

has a second solution manifold $\tilde{w}(\epsilon, \chi), \epsilon \geq 0, \chi \in \mathbf{g}_{(\bar{\sigma}, \bar{\mu})}^{*}$ to the parameters $\tilde{\nu}(\epsilon, \chi)=\chi$ and $E=\tilde{E}(\epsilon, \chi)$ such that $w(0, \chi)=w(\chi)[12]$. Hence, we obtain an $(r+1)$-dimensional family of fixed points $\tilde{v}(\epsilon, \chi):=(\tilde{\nu}, \tilde{w}, \tilde{E})(\epsilon, \chi)$ of $\Pi_{\tilde{\mathcal{N}}}^{2}$, which gives rise to a family $\tilde{x}(\epsilon, \chi)$ of points on RPOs $\tilde{\mathcal{P}}(\epsilon, \chi)$ with relative period $\tilde{\tau}(\epsilon, \chi)$, drift symmetry $\tilde{\sigma}(\epsilon, \chi)$, momentum $\tilde{\mu}(\epsilon, \chi)=\bar{\mu}+\chi$, and energy $\tilde{E}(\epsilon, \chi)$ such that $\tilde{\tau}(0,0)=2 \bar{\tau}, \tilde{\sigma}(0,0)=\bar{\sigma}^{2}, \tilde{E}(0,0)=\bar{E}$.

From $\tilde{\sigma}(\epsilon, \chi) \tilde{\mu}(\epsilon, \chi)=\tilde{\mu}(\epsilon, \chi)$ (as we saw in $(2.15)$ ) we conclude that $\tilde{\sigma}(\epsilon, \chi) \in \tilde{\Gamma}_{\tilde{\mu}(\epsilon, \chi)}$. Since $\tilde{\mathcal{N}}_{0} \simeq \mathbf{g}_{(\bar{\sigma}, \bar{\mu})}^{*}$ is invariant under $\tilde{\Gamma}_{\bar{\mu}}$ we know that $\tilde{\Gamma}_{\tilde{\mu}(\epsilon, \chi)} \subseteq \tilde{\Gamma}_{\bar{\mu}}=\Gamma_{(\bar{\sigma}, \bar{\mu})}$ and, therefore, $\tilde{\sigma}(\epsilon, \chi) \in$ 
$\Gamma_{(\bar{\sigma}, \bar{\mu})}$. Since, by Remark 2.17, $\Gamma_{(\bar{\sigma}, \bar{\mu})}^{\mathrm{id}}$ is abelian and $\mathbf{g}_{(\bar{\sigma}, \bar{\mu})} \subseteq \mathbf{g}_{\alpha}$, we can decompose $\tilde{\sigma}(\epsilon, \chi)=$ $\alpha^{2} \exp (-\tilde{\tau}(\epsilon, \chi) \tilde{\xi}(\epsilon, \chi))$, where $\tilde{\xi}(\epsilon, \chi) \in \mathbf{g}_{(\bar{\sigma}, \bar{\mu})}, \operatorname{Ad}_{\alpha} \tilde{\xi}(\epsilon, \chi)=\tilde{\xi}(\epsilon, \chi)$, and $\operatorname{Ad}_{\alpha}^{*} \chi=\chi$.

Moreover, from [12] we have $\mathrm{D}_{\epsilon} \tilde{w}(0,0)=\bar{w}$. Since $\tilde{v}(-\epsilon, \chi):=\Pi_{\mathcal{N}_{1}}(\tilde{v}(\epsilon, \chi))$ lies on the $\operatorname{RPO} \tilde{\mathcal{P}}(\epsilon, \chi)$ too, we have $\tilde{\tau}(\epsilon, \chi)=\tilde{\tau}(-\epsilon, \chi)$. By energy conservation $\tilde{E}(\epsilon, \chi)=\tilde{E}(-\epsilon, \chi)$, and so the first three statements of $(3.2)$ hold. Let $\tilde{v}(-\epsilon, \chi) \simeq \tilde{x}(-\epsilon, \chi) \in \mathcal{S}$. By the definition (2.17) of $\Pi$ for the symmetry group $\tilde{\Gamma}=\Gamma_{\bar{\sigma}}, \tilde{\sigma}(\epsilon, \chi)=\gamma(\tilde{x}(-\epsilon, \chi)) \gamma(\tilde{x}(\epsilon, \chi))$ and $\tilde{\sigma}(-\epsilon, \chi)=$ $\gamma(\tilde{x}(\epsilon, \chi)) \gamma(\tilde{x}(-\epsilon, \chi))$. By Lemma 2.18 b) we have $\gamma(\tilde{x}( \pm \epsilon, \chi)) \in \alpha \Gamma_{(\bar{\sigma}, \tilde{\mu})}^{\mathrm{id}}$. Therefore, by Remark $2.17, \tilde{\sigma}(-\epsilon, \chi)=\tilde{\sigma}(\epsilon, \chi)$. This proves the last two equations of (3.2).

Remark 3.3 If $\mathrm{D}_{E} \psi(0, \bar{E})=0, \mathrm{D}_{\chi_{1}} \psi(0, \bar{E}) \neq 0$, then Theorem 3.2 remains valid if we change the parametrization from $(\epsilon, \chi)$ to $(\epsilon, \eta)$, where $\eta=\left(\chi_{2}, \ldots, \chi_{r}, e\right)$ and $e=E-\bar{E}$. In this case the component $\tilde{\nu}_{1}(\epsilon, \eta)$ of the bifurcating RPOs through $\tilde{x}(\epsilon, \eta) \simeq(\tilde{\nu}(\epsilon, \eta), \tilde{w}(\epsilon, \eta), E(\epsilon, \eta))$ on the slice $\tilde{\mathcal{N}}$ and the momentum component $\tilde{\mu}_{1}(\epsilon, \eta)$ of the momentum $\tilde{\mu}(\epsilon, \eta)=\bar{\mu}+\tilde{\nu}(\epsilon, \eta)$ of the bifurcating RPO through $\tilde{x}(\epsilon, \eta)$ depend nonlinearly on $(\epsilon, \eta), \mathrm{D}_{\epsilon} \tilde{\mu}_{1}(0,0)=0, \tilde{\nu}_{j}(\epsilon, \eta)=\chi_{j}$, $j=2, \ldots, r$, and $\tilde{E}(\epsilon, \eta)=\bar{E}+e$.

Remark 3.4 In the co-moving frame $\bar{\xi}$ a relative period doubling bifurcation at $\bar{x}$ becomes a flip-doubling bifurcation or a flip-pitchfork bifurcation of the corresponding $\bar{T}=\ell \bar{\tau}$-periodic orbit through $\bar{x}[5,23]$. The drift symmetry of the bifurcating periodic orbit in the co-moving frame is $\tilde{\alpha}=\alpha^{2}$. If $\ell$ is even then its period in the co-moving frame is $\tilde{T} \approx \bar{T}$ and its spatio-temporal symmetry group is broken to $\mathbb{Z}_{\tilde{\ell}}$ with $\tilde{\ell}=\ell / 2$. This scenario is called a flip-pitchfork bifurcation in [5]. If $\ell$ is odd then its period in the co-moving frame is $\tilde{T} \approx 2 \bar{T}$ and its spatio-temporal symmetry group remains $\mathbb{Z}_{\tilde{\ell}}$ with $\tilde{\ell}=\ell$. This scenario is called flip-doubling bifurcation in [5].

Remark 3.5 The assumption $r_{(\bar{\sigma}, \bar{\mu})}=r_{\left(\bar{\sigma}^{2}, \bar{\mu}\right)}$ implies that the block $M_{0}=\left.\operatorname{Ad}_{\bar{\sigma}^{-1}}^{*}\right|_{\mathbf{g}_{\mu}^{*}}$ in the linearization $M=\mathrm{D}_{x} \bar{\sigma} \Phi^{\bar{\tau}}(\bar{x})$ of the RPO at the bifurcation point does not have an eigenvalue -1 , see Proposition 2.19. Therefore, the eigenvalue -1 of $\mathrm{D} \Pi(\bar{x})$ has algebraic multiplicity two. Generically, the drift symmetry $\bar{\sigma}^{2}$ of the bifurcating RPO at the bifurcation point is regular. In this case, $\bar{\sigma}$ is regular too, and the above assumption reads $r_{\bar{\sigma}^{2}}=r_{\bar{\sigma}}$. Then $\operatorname{Ad}_{\bar{\sigma}}$ does not have an eigenvalue -1 either, and the eigenvalue -1 of $M$ has algebraic multiplicity two as well. If $\bar{\sigma}^{2}$ is not regular then $M$ might have additional eigenvalues -1 .

If we continue in energy while fixing the value of the momentum map as in (2.28), we get the following corollary:

Corollary 3.6 Under the assumptions of Theorem 3.2, if $\mathrm{D}_{E} \psi(\bar{E}, 0) \neq 0$, there is a smooth path $\tilde{x}(\epsilon) \in \mathcal{X}^{\bar{\mu}}$ with $\tilde{x}(0)=\bar{x}, \epsilon \geq 0$, such that $\tilde{x}(\epsilon)$ lies on an $R P O \tilde{\mathcal{P}}(\epsilon)$ of $(2.1)$ with relative period $\tilde{\tau}(\epsilon)$, drift symmetry $\tilde{\sigma}(\epsilon)=\alpha \exp (-\tilde{\tau}(\epsilon) \tilde{\xi}(\epsilon)) \in \Gamma_{(\bar{\sigma}, \bar{\mu})}$ at $\tilde{x}(\epsilon)$, energy $\tilde{E}(\epsilon)$ and momentum $\bar{\mu}$, where $\tilde{x}(0)=\bar{x}, \tilde{\tau}(0)=2 \bar{\tau}, \tilde{\xi}(0)=\bar{\xi}, \tilde{E}(0)=\bar{E}, \tilde{E}^{\prime}(0)=0, \tilde{\xi}^{\prime}(0)=0, \tilde{x}^{\prime}(0)=\bar{w}$.

Similarly, if we continue in the momentum component $\mu_{1}$ while fixing the other momentum components and the energy as in (2.29), we get the following corollary:

Corollary 3.7 In the setting of Remark 3.3, i.e., if $\mathrm{D}_{\bar{\chi}_{1}} \psi(\bar{E}, 0) \neq 0$, there is a smooth path $\tilde{x}(\epsilon) \in \mathcal{X}^{\bar{E}, \bar{\mu}^{b}}$ with $\tilde{x}(0)=\bar{x}$ such that $\tilde{x}(\epsilon)$ lies on an $R P O \tilde{\mathcal{P}}(\epsilon)$ of $(2.1), \epsilon \geq 0$, with relative period $\tilde{\tau}(\epsilon)$, drift symmetry $\tilde{\sigma}(\epsilon)=\alpha \exp (-\tilde{\tau}(\epsilon) \tilde{\xi}(\epsilon)) \in \Gamma_{(\bar{\sigma}, \bar{\mu})}$ at $\tilde{x}(\epsilon)$, energy $\overline{\bar{E}}$ and momentum $\tilde{\mu}(\epsilon)=\left(\tilde{\mu}_{1}(\epsilon), \bar{\mu}^{b}\right)$, where $\tilde{x}(0)=\bar{x}, \tilde{\tau}(0)=\bar{\tau}, \tilde{\xi}(0)=\bar{\xi}, \tilde{\mu}_{1}(0)=\bar{\mu}_{1}, \tilde{\mu}_{1}^{\prime}(0)=0, \tilde{\xi}^{\prime}(0)=0$, $\tilde{x}^{\prime}(0)=\bar{w}$. 


\subsection{Detection of relative period doubling bifurcations}

Assume as before that $\bar{x}$ is a relative period doubling bifurcation point and that $\mathrm{D}_{w} \Pi_{\mathcal{N}_{1}}(0,0, \bar{E})$ has an eigenvalue -1 with multiplicity 2 . Let $\mathcal{P}(c)$ be a branch of RPOs with $C(x)=\mathbf{J}_{1}(x)$ or $C(x)=H(x)$ as in Corollaries 2.29 and 2.30 above. Choose coordinates on the Poincaré section $\mathcal{S}$ at $\bar{x}$ as in Lemma 2.18 and let $x(c) \simeq(\nu(c), w(c), E(c)) \in \mathcal{N}$ be such that $x(\bar{c})=\bar{x}$.

Lemma 3.8 Generically, at a relative period doubling bifurcation point $\bar{x}$ along a path of RPOs $x(c) \in \mathcal{P}(c)$, the determinant $\operatorname{det}\left(\mathrm{D}_{w} \Pi_{\mathcal{N}_{1}}((\nu, w, E)(c))+\mathrm{id}_{\mathcal{N}_{1}}\right)$ changes sign.

Proof. Let $M_{1}(c):=\mathrm{D}_{w} \Pi_{\mathcal{N}_{1}}((\nu, w, E)(c))$. Under the above assumptions, the pair $\lambda_{1,2}(c)$ of eigenvalues of $M_{1}(c)$ with $\lambda_{1,2}(\bar{c})=-1$ generically lies on the unit circle before collision and on the real axis after collision or vice versa, as we saw in Remark 3.1. Let $B(c)=\left.M_{1}(c)\right|_{\mathcal{Y}(c)}$, where $\mathcal{Y}(c)$ is the generalized eigenspace of $M_{1}(c)$ to the eigenvalues $\lambda_{1,2}(c)$ with $\lambda_{1,2}(\bar{c})=-1$. Then, $\lambda_{1}(c)=-1+a(c), \lambda_{2}(c)=1 /(-1+a(c))$ for $c \leq \bar{c}$ with $a(c) \in \mathbb{R}, a(\bar{c})=0$, and $\lambda_{1,2}(c)=\mathrm{e}^{ \pm \mathrm{i} \phi(c)}$ with $\phi(c) \in \mathbb{R}, \phi(\bar{c})=\pi$, for $c \geq \bar{c}$, or vice versa. Consequently, $\operatorname{det}(B(c)+\mathrm{id})=$ $-a^{2}(c)+O\left(a^{3}(c)\right)<0$ for $c<\bar{c}$ and

$$
\operatorname{det}\left(B(c)+\mathrm{id}_{2}\right)=\left(\mathrm{e}^{\mathrm{i} \phi(c)}+1\right)\left(\mathrm{e}^{-\mathrm{i} \phi(c)}+1\right)=2(1+\cos \phi(c))>0 \text { for } c>\bar{c},
$$

or vice versa. Since the other eigenvalues of $M_{1}(c)$ are bounded away from -1 near $c=\bar{c}$, we conclude that $\operatorname{det}\left(M_{1}(c)+\operatorname{id}_{\mathcal{N}_{1}}\right)$ also changes sign at $c=\bar{c}$.

Under the assumptions of Theorem 3.2 also $\operatorname{det}\left(\mathrm{D} \Pi(x(c))+\mathrm{id}_{\mathcal{N}}\right)$ changes sign at $c=\bar{c}$, as we saw in Remark 3.5; hence, in a manner similar to the case of dissipative systems with discrete symmetry groups [23], relative period doubling bifurcations can be detected by a sign change of

$$
d(y)=\operatorname{det}\left(\mathrm{P}_{\mathcal{N}} \mathrm{D}_{x} \alpha \Phi^{\tau}(x ; \xi) \mathrm{P}_{\mathcal{N}}+\mathrm{id}_{\mathcal{N}}\right) .
$$

Here, $\mathrm{P}_{\mathcal{N}}$ is the orthogonal projection from the phase space $\mathcal{X}$ to the tangent space $\mathcal{N}$ of the Poincaré section at $\bar{x}$ with kernel $\mathcal{T}_{\bar{x}} \overline{\mathcal{P}}$. Recall that $\mathrm{D}_{x} \alpha \Phi^{\tau}(x ; \xi)$ is related to the derivative of the shooting equations, see Remark 2.27.

Remark 3.9 Note that the trace condition (3.1) is not suitable for the numerical detection of relative period-doubling bifurcations (except, of course, in the case that $\operatorname{dim} \mathcal{X}=2$ ). Since the eigenvalue -1 generically has algebraic multiplicity two at bifurcation, see Remark 3.5, we could remove the projection $\mathrm{P}_{\mathcal{N}}$ completely from the test function $d(y)$ in $(3.3)$, or replace it with the projection onto $\widetilde{\mathcal{N}}_{1}$ with kernel $\mathbf{g}_{\sigma} \bar{x} \oplus \operatorname{span}\left(f_{H}(\bar{x})\right) \oplus \widetilde{\mathcal{N}}_{0} \oplus \widetilde{\mathcal{N}}_{2}$. Here, $\widetilde{\mathcal{N}}=\widetilde{\mathcal{N}}_{0} \oplus \widetilde{\mathcal{N}}_{1} \oplus \widetilde{\mathcal{N}}_{2}$ is the normal space for the symmetry group $\tilde{\Gamma}=\Gamma_{\bar{\sigma}}$ from Lemma 2.18. From a theoretical point of view, c.f. Lemma 3.8, a projection onto $\mathcal{N}_{1}$ is possible as well. However, the dimension of $\mathcal{N}_{1}$ varies along the branch of RPOs, if a non-regular momentum value is passed, and this may cause numerical instability. Note that the dimension of $\mathcal{N}$ is constant along the branch of RPOs as all RPOs are assumed to have trivial isotropy. Moreover, since $\bar{\mu}$ is assumed to be a regular momentum for the group $\tilde{\Gamma}=\Gamma_{\bar{\sigma}}$, the dimension of $\widetilde{\mathcal{N}}_{1}$ is also locally constant.

\subsection{Computation of relative period doubling points and branch switch- ing}

Relative period doubling bifurcations, which satisfy the assumptions of Theorem 3.2, can be computed similarly to period doubling bifurcations of dissipative systems with discrete symmetry groups, see, e.g., [9, 23], by subdivision of (3.3) along a family of solutions $y(c)$ of $(2.28)$ or $(2.29)$, respectively. 
Once a relative period doubling point $\bar{y}=(\bar{x}, \bar{\tau}, \bar{\xi}, 0)$ on the original branch has been found, the starting point $\tilde{y}=(\tilde{x}, \tilde{T}, \tilde{\xi}, 0)$ for the bifurcating branch has to be computed. We set $\tilde{T}=\bar{T}$, $\tilde{\ell}=\ell / 2$ at the bifurcation point for a relative flip pitchfork bifurcation (as defined in Remark $3.4)$ and $\tilde{T}=2 \bar{T}, \tilde{\ell}=\ell$ otherwise, and let, $\tilde{\alpha}=\alpha^{2}, \tilde{\xi}=\bar{\xi}$.

We compute the tangent $\tilde{t}$ for the bifurcating branch as follows: At a relative period doubling bifurcation the matrix $M$ in (2.19) has an eigenvalue -1 which is of algebraic multiplicity two if $\operatorname{Ad}_{\bar{\sigma}}$ does not have -1 as an eigenvalue. This condition is generically satisfied (Remark 3.5). Let $w$ span the kernel of $M+$ id. We compute that

$$
\mathrm{D} H(\bar{x}) w=\mathrm{D} H(\bar{x}) M w=-\mathrm{D} H(\bar{x}) w=0 .
$$

Moreover,

$$
\mathrm{DJ}_{e_{\xi}^{j}}(\bar{x}) w=0, \quad j=1, \ldots, r_{\bar{\mu}},
$$

since $\left.\operatorname{Ad}_{\bar{\sigma}}^{*}\right|_{\mathbf{g}_{\mu}^{*}}$ does not have -1 as an eigenvalue. Then, by Proposition 2.19 applied to the symmetry group $\tilde{\Gamma}=\Gamma_{\bar{\sigma}}$, projecting $w$ orthogonal to $\operatorname{span}(f(\bar{x})) \oplus \mathbf{g}_{(\bar{\sigma}, \bar{\mu})} \bar{x}$ we obtain the eigenvector $\bar{w}$ of $\mathrm{D}_{\tilde{\mathcal{N}}_{1}}(0)$ to the eigenvalue -1 . Let $\tilde{t}=\left(\tilde{t}_{x}, \tilde{t}_{T}, \tilde{t}_{\xi}, \tilde{t}_{\lambda_{E}}, \tilde{t}_{\lambda_{\mu}}\right)$ denote the continuation tangent for the bifurcating branch. Clearly, $\tilde{t}_{\lambda_{E}}=0, \tilde{t}_{\lambda_{\mu}}=0$. By Theorem 3.2, in particular (3.2), the bifurcating family of RPOs $\tilde{\mathcal{P}}(\epsilon, \chi)$ satisfies $\mathrm{D}_{\epsilon} \tilde{x}(0)=\bar{w}, \mathrm{D}_{\epsilon} \tilde{\tau}(0)=0$ and $\mathrm{D}_{\epsilon} \tilde{\xi}(0)=0$ so that $\tilde{t}_{x}=\bar{w}, \tilde{t}_{T}=0, \tilde{t}_{\xi}=0$.

Remark 3.10 Note that (3.3) can also change sign if $\operatorname{Ad}_{\bar{\sigma}}$ passes through an eigenvalue -1 , and this can also happen for the modified test functions discussed in Remark 3.9. Therefore, once a relative period doubling point $\bar{x}$ has been computed it should be checked whether $\bar{w}$ is linearly independent of $\mathbf{g} \bar{x}$ and whether (3.4) is satisfied. If either of those conditions is violated then, by Proposition 2.19, the eigenvalue -1 of $M$ is due to an eigenvalue -1 of $\operatorname{Ad}_{\bar{\sigma}}$.

Remark 3.11 In the multiple shooting context, see Remark 2.31, it is natural to double the number of multiple-shooting points on the bifurcating branch. The second half of $\tilde{x}=$ $\left(\tilde{x}_{1}, \ldots, \tilde{x}_{2 k}\right)$ is computed as

$$
\tilde{x}_{i}=\bar{x}_{i} \quad \text { for } \quad i=1, \ldots, k, \quad \tilde{x}_{i+k}=\alpha^{-1} \bar{x}_{i} \quad \text { for } \quad i=1, \ldots, k .
$$

The continuation tangent $\tilde{t}=\left(\tilde{t}_{x}, 0,0,0,0,0\right)$ of the bifurcating branch is computed in a similar way: we compute the eigenvector $w$ to the eigenvalue -1 of $M=\mathrm{D}_{x} \bar{\sigma} \Phi^{\bar{\tau}}(\bar{x})$ (see Remark 2.27) and set

$$
t_{1}=w, t_{i+1}=G_{i} t_{i}, \quad i=1, \ldots, k-1, \quad t_{i+k}=-\alpha^{-1} t_{i}, \quad i=1, \ldots, k-1 .
$$

Here the matrices

$$
G_{i}=\mathrm{D}_{x_{i}} \Phi^{\frac{\Delta s_{i} T}{\ell}}\left(x_{i} ; \lambda\right), \quad i=1,2, \ldots, k-1, \quad G_{k}=\mathrm{D}_{x_{k}} \alpha \Phi^{\frac{\Delta s_{k} T}{\ell}}\left(x_{k} ; \lambda\right)
$$

are available as derivative of (2.25) if, for example, a Gauss-Newton method is used to solve (2.25). Let $F: \mathcal{X}^{k} \times \mathbb{R}^{r+q+2} \rightarrow \mathcal{X}^{k} \times \mathbb{R}^{q}$, be given by (2.28) or (2.29). Define $t^{f}, t^{\xi_{1}}, \ldots, t^{\xi_{r}}$ as in Remark 2.31. Define $\tilde{t}^{f}, \tilde{t}^{\xi_{j}} \in \mathcal{X}^{2 k} \times \mathbb{R}^{r+q+2}, j=1, \ldots r$, as

$$
\tilde{t}_{i}^{f}=t_{i}^{f}, \quad \tilde{t}_{i}^{\xi_{j}}=t_{i}^{\xi_{j}}, \quad \tilde{t}_{i+k}^{f}=\alpha^{-1} t_{i}^{f}, \quad \tilde{t}_{i+k}^{\xi_{j}}=\alpha^{-1} t_{i}^{\xi_{j}}, \quad i=1, \ldots, k,
$$

and set $\tilde{t}_{T}^{f}=\tilde{t}_{T}^{\xi_{j}}=\tilde{t}_{\lambda_{E}}^{f}=\tilde{t}_{\lambda_{E}}^{\xi_{j}}=0, \tilde{t}_{\xi}^{f}=\tilde{t}_{\xi}^{\xi_{j}}=\tilde{t}_{\lambda_{\mu}}^{f}=\tilde{t}_{\lambda_{\mu}}^{\xi_{j}}=0$. Projecting $t$ orthogonally to $\tilde{t}^{f}, \tilde{t}^{\xi_{1}}, \ldots, \tilde{t}^{\xi_{r}}$ we obtain a continuation tangent for the bifurcating branch. 


\subsection{Detection and computation of relative period halving bifurcations}

In this section we show how relative period halving bifurcations, which occur along branches of RPOs defined by (2.28) or (2.29) and satisfy the assumptions of Corollary 3.6 or Corollary 3.7, respectively, can be detected and computed numerically.

As in the case of dissipative systems with finite symmetry group discussed in [23], we compute all choices of $\tilde{\alpha}$ such that $\tilde{\alpha}^{2}=\alpha$, and at each solution $y=(x, T, \xi, 0)$ of $(2.28)$ or $(2.29)$, respectively, we compute for each choice of $\tilde{\alpha}$,

$$
u(y):=\tilde{\alpha} \Phi^{\frac{T}{2 \ell}}(x ; \xi)-x .
$$

A relative period halving bifurcation is detected, if

$$
\left\langle u^{(0)}, u^{(1)}\right\rangle<0, \quad u^{(0)}=u\left(y^{(0)}\right), \quad u^{(1)}=u\left(y^{(1)}\right),
$$

at two consecutively computed solutions $y^{(0)}=\left(x^{(0)}, T^{(0)}, \xi^{(0)}, 0\right)$ and $y^{(1)}=\left(x^{(1)}, T^{(1)}, \xi^{(1)}, 0\right)$ of (2.28) or (2.29), respectively. This can be seen as follows: By Corollary 3.6 or Corollary 3.7, respectively, the functions $x(\epsilon), \xi(\epsilon)$ and $\tau(\epsilon)=T(\epsilon) / \ell$ are differentiable at $\epsilon=0$. Moreover at the relative period halving point, $\left.u(y(\epsilon))\right|_{\epsilon=0}=0$ and $\left.\mathrm{D}_{\epsilon} u(y(\epsilon))\right|_{\epsilon=0}=-2 \bar{w} \neq 0$.

By Corollary 3.6 or Corollary 3.7, respectively, at the relative period halving bifurcation point we have $\left.\mathrm{D}_{\epsilon} C(x(\epsilon))\right|_{\epsilon=0}=0$. Therefore, once a relative period halving bifurcation has been detected between $y^{(0)}$ and $y^{(0)}$, an initial guess $\hat{y}$ for the bifurcation point can be computed by using interpolation $(y(\tau), c(\tau))$ between $\left(y^{(0)}, C\left(x^{(0)}\right)\right.$ and $\left(y^{(1)}, C\left(x^{(1)}\right)\right.$ to find a critical value $\hat{c}=C(\hat{y})$ of $c(\tau)$. Then the bifurcation point can be computed by switching to the branch of RPOs with halved relative period and using the methods for relative period doubling bifurcations from the previous section on this branch. This is similar to the case of dissipative systems with finite symmetry groups [23].

Since we assume (see Theorem 3.2) that the relative period halving point has a regular driftmomentum pair $(\bar{\sigma}, \bar{\mu})$, by Remark 2.17 the sub-algebra $\mathbf{g}_{(\bar{\sigma}, \bar{\mu})}$ is abelian. Therefore $\Gamma_{(\bar{\sigma}, \bar{\mu})}^{\mathrm{id}}$ is isomorphic to $\mathbb{T}^{n}$ and is a subgroup of $Z(\alpha)$. Here, $\mathbb{T}^{n}=\left(S^{1}\right)^{n}$ is an $n$-dimensional torus. The group $L=\Gamma_{(\bar{\sigma}, \bar{\mu})} \cap Z\left(\Gamma_{(\bar{\sigma}, \bar{\mu})}^{\mathrm{id}}\right) / \Gamma_{(\bar{\sigma}, \bar{\mu})}^{\mathrm{id}}$ is finite because $\Gamma$ is assumed to be compact. Since $r_{\left(\bar{\sigma}^{2}, \bar{\mu}\right)}=r_{(\bar{\sigma}, \bar{\mu})}$ in Theorem 3.2, all possible choices of square roots $\tilde{\alpha}$ of $\alpha$ lie in $Z\left(\Gamma_{(\bar{\sigma}, \bar{\mu})}^{\mathrm{id}}\right)$. So after reduction by $\Gamma_{(\bar{\sigma}, \bar{\mu})}^{\mathrm{id}}$, we are back to the case of finite groups $L$ treated in [23].

Assume that $\alpha$ has a square root $\tilde{\alpha} \in L: \tilde{\alpha}^{2}=\alpha$. Then $\alpha$ has indeed two square roots in $\mathbb{Z}_{2 \ell}(\tilde{\alpha}) \subseteq L$, namely, $\tilde{\alpha}_{1}=\tilde{\alpha}$ and $\tilde{\alpha}_{2}=\tilde{\alpha}^{\ell+1}$. In the case of continuous symmetry groups $\Gamma$ the test $(3.5)$ for relative period halving bifurcations has to be used for all $\tilde{\alpha}=\tilde{\alpha}_{1,2} \exp (\xi)$, where $\xi \in \mathbf{t}^{n}$ is such that $\xi_{j}=0$ or $\xi_{j}=\pi, j=1, \ldots, n$. Here, $\mathbf{t}^{n}$ is the Lie algebra of $\mathbb{T}^{n}$. For example, if $\Gamma_{(\bar{\sigma}, \bar{\mu})}^{\mathrm{id}}=\mathrm{SO}(2)$, and $R_{\phi}$ is a rotation by $\phi$, then $\exp (\xi)=$ id or $\exp (\xi)=R_{\pi}$.

\section{Application to rotating choreographies}

In this section we apply our methods for numerical bifurcations of RPOs to rotating choreographies of the three-body problem. Using the software package SYMPERCON [18] we find that the type II family of rotating choreographies undergoes a symmetry-increasing flip pitchfork bifurcation in the co-rotating frame to the type I family of rotating choreographies. We also report on several relative period doubling bifurcations and a turning point of the planar (type III) family of rotating choreographies. 


\subsection{N-body problems and their symmetries}

We consider the motion of $N$ identical bodies of mass 1 in $\mathbb{R}^{3}$ subject to internal forces they exert on each other. We assume that these forces are given by $\frac{1}{2} N(N-1)$ identical copies of a potential energy function $V$ (one for each pair of bodies), which depends only on the distance between the bodies. Writing $p_{j}$ for the momenta conjugate to the positions $q_{j}, q=\left(q_{1}, \ldots, q_{N}\right)$, $p=\left(p_{1}, \ldots, p_{N}\right)$, the Hamiltonian is

$$
H(q, p)=\frac{1}{2} \sum_{j=1}^{N}\left|p_{j}\right|^{2}+\sum_{i<j} V\left(r_{i j}\right), \quad \text { where } \quad r_{i j}=\left|q_{i}-q_{j}\right| \text { and } V(r)=-\frac{1}{r} .
$$

Excluding collisions, the configuration space $\mathcal{Q}$ is

$$
\mathcal{Q}=\left\{q=\left(q_{1}, \ldots, q_{N}\right) \in \mathbb{R}^{3 N}, \quad q_{i} \neq q_{j} \text { for } i \neq j\right\}
$$

and the phase space is $\mathcal{Q} \times \mathbb{R}^{3 N} \subset \mathbb{R}^{6 N}$. The equations of motion are

$$
\dot{q}_{j}=p_{j}, \quad \dot{p}_{j}=\sum_{i \neq j} \frac{q_{i}-q_{j}}{r_{i j}^{3}}, \quad j=1, \ldots, N,
$$

and the angular momentum is $\mathbf{J}(q, p)=\sum_{j=1}^{N} q_{j} \times p_{j}$. Without loss of generality, the centre of mass of the systems can be assumed to be fixed at 0 restricting the configuration space to

$$
\mathcal{Q}^{0}=\left\{q \in Q: \sum_{j=1}^{N} q_{j}=0\right\}
$$

with corresponding phase space $\mathcal{X}=\mathcal{Q}^{0} \times \mathbb{R}^{3(N-1)} \subseteq \mathbb{R}^{6(N-1)}$.

The $N$-identical-body Hamiltonian (4.1) has the following symmetries:

1. Rotations and reflections of $\mathbb{R}^{3}$ : These form the orthogonal group $\mathrm{O}(3)$, which acts diagonally on the positions and velocities:

$$
R\left(q_{1}, \ldots, q_{N}, p_{1}, \ldots, p_{N}\right)=\left(R q_{1}, \ldots, R q_{N}, R p_{1}, \ldots, R p_{N}\right), \quad R \in \mathrm{O}(3), q_{j}, p_{j} \in \mathbb{R}^{3} .
$$

In the following let $\kappa_{i} \in \mathrm{O}(3)$ be the reflection satisfying $\kappa_{i} e^{i}=-e^{i}, \kappa_{i} e^{j}=e^{j}$ for $j \neq i$, $i, j=1,2,3$. We denote by $R_{j}(\phi)$ a rotation around the $e^{j}$-axis by the angle $\phi$.

2. Permutations of identical bodies: Because we assume that all the bodies are identical the Hamiltonian is also invariant under the action of $S_{N}$, the group of all permutations of the integers $1, \ldots, N$ :

$$
\pi\left(q_{1}, \ldots, q_{N}, p_{1}, \ldots, p_{N}\right)=\left(q_{\pi(1)}, \ldots, q_{\pi(N)}, p_{\pi(1)}, \ldots, p_{\pi(N)}\right) \quad \pi \in S_{N}, q_{j}, p_{j} \in \mathbb{R}^{3} .
$$

We frequently use the notation $\pi=\left(\pi^{-1}(1), \ldots, \pi^{-1}(N)\right)$.

Taken together these three symmetry groups give a symplectic action of

$$
\Gamma=\mathrm{O}(3) \times S_{N}
$$

on $\mathcal{X}$, which leaves the Hamiltonian (4.1) invariant.

The three-body problem also has the scaling symmetry

$$
x(t)=(q(t), p(t)) \rightarrow\left(c q\left(c^{-3 / 2} t\right), c^{-1 / 2} p\left(c^{-3 / 2} t\right)\right),
$$

and this will be used in Section 4.4. 


\subsection{Rotating choreographies of the three body problem}

As in [3] we define:

Definition 4.1 A periodic orbit of (4.2) is called a choreography if all the bodies follow the same path in $\mathbb{R}^{3}$, separated only by a phase shift. This is equivalent to requiring that the spatio-temporal symmetry group of the periodic orbit contains an order $N$ cyclic permutation $\pi \in S_{N}$ which can always be taken to act on $\mathcal{Q}$ by $\pi q=\left(q_{2}, q_{3}, \ldots, q_{N}, q_{1}\right)$. Similarly, a relative periodic orbit of (4.2) with angular velocity $\xi$ is a rotating choreography if it is a choreography in coordinates rotating with velocity $\xi$.

The famous Figure Eight of Chenciner and Montgomery [4] is a choreography of the $N$ identical-body system (4.2) with $N=3$. Let $\left\{e^{1}, e^{2}, e^{3}\right\}$ be a fixed orthogonal set of axes in $\mathbb{R}^{3}$ and assume that the eight lies in the plane perpendicular to $e^{3}$ aligned along the $e^{1}$-axis with both $e^{2}$-axis and $e^{1}$-axis as symmetry axis. The spatial symmetry group of the Figure Eight choreography is then the group

$$
K=\mathbb{Z}_{2}=\left\langle\kappa_{3}\right\rangle
$$

generated by $\kappa_{3}$, a reflection about the $\left(e^{1}, e^{2}\right)$-plane containing the Figure Eight. The drift symmetry $\alpha:=\kappa_{1}(231)$ of the eight is a reflection in the $\left\{e^{1}, e^{2}\right\}$-plane composed with a cyclic permutation of the bodies and has order $\ell=6$. Fix some point $\bar{x}$ on the Figure Eight, let $\bar{\tau}$ be its relative period, $\bar{\mu}=0$ its momentum and $\bar{E}=H(\bar{x})$ be its energy.

As shown by Chenciner et al. [3] three families of rotating choreographies bifurcate from the Figure Eight when angular momentum is switched on:

I. The type I family of rotating eights $\mathcal{P}_{I}(E, \nu)$ rotates around the $e^{1}$-axis. The drift symmetry in the rotating frame at $x_{I}(E, \nu) \in \mathcal{P}_{I}(E, \nu)$, where $x_{I}(E, \nu)$ is a smooth function, $x_{I}(\bar{E}, 0)=\bar{x}$, is $\alpha_{I}=\kappa_{1}(231)$ and has order $\ell_{I}=6$. Moreover $H\left(x_{I}(E, \nu)\right)=E$, $\mathbf{J}\left(x_{I}(E, \nu)\right)=(\nu, 0,0)^{T}$. The relative periods $\tau_{I}(E, \nu)$ of the bifurcating RPOs are close to the relative period of the original Figure Eight and $\tau_{I}(\bar{E}, 0)=\bar{\tau}$.

II. The type II rotating eights $\mathcal{P}_{I I}(E, \nu)$ rotate around the $e^{2}$-axis. The drift symmetry in the rotating frame at $x_{I I}(E, \nu) \in \mathcal{P}_{I}(E, \nu)$, where $x_{I I}(E, \nu)$ is a smooth function, $x_{I I}(\bar{E}, 0)=$ $\bar{x}$, is $\alpha_{I I}=\kappa_{1} \kappa_{3}(231)$ and has order $\ell_{I I}=6 . H\left(x_{I I}(E, \nu)\right)=E, \mathbf{J}\left(x_{I I}(E, \nu)\right)=(0, \nu, 0)^{T}$. The relative period $\tau_{I I}(E, \nu)$ of the bifurcating RPOs satisfies $\tau_{I I}(\bar{E}, 0)=\bar{\tau}$.

III. The type III rotating eights $\mathcal{P}_{I I I}(E, \nu)$ are planar, i.e., have spatial symmetry $K=\left\langle\kappa_{3}\right\rangle$ at $x_{I I I}(E, \nu) \in \mathcal{P}_{I}(E, \nu)$, where $x_{I I I}(E, \nu)$ is a smooth function, $x_{I I I}(\bar{E}, 0)=\bar{x}$. The drift symmetry in the rotating frame at $x_{I I I}(E, \nu)$ is $\alpha_{I I I}=(312)$ and has order $\ell_{I I I}=3$. $H\left(x_{I I I}(E, \nu)\right)=E, \mathbf{J}\left(x_{I I I}(E, \nu)\right)=(0,0, \nu)^{T}$. The relative period $\tau_{I I I}(E, \nu)$ of the family of bifurcating RPOs doubles at the bifurcation point: $\tau_{I I I}(\bar{E}, 0)=2 \bar{\tau}$.

\subsection{Flip up pitchfork bifurcation of the type II rotating eight}

We fix the energy to the value $\bar{E}=-1.287$ of the original Figure Eight. Along the branch $\mathcal{P}(s)$ of rotating eights of type II with drift symmetry $\sigma(s)=\exp (-\tau(s) \xi(s)) \alpha$ at $x(s) \in \mathcal{P}(s)$, where $\alpha=\alpha_{I I}=(231) R_{2}(\pi)$ is of order $\ell=6$, using SYMPERCON we then find a symmetry-increasing pitchfork bifurcation in the co-rotating frame. The emanating RPOs $\tilde{\mathcal{P}}(s)$ have halved relative period and drift symmetry $\tilde{\sigma}(s)=\exp (-\tilde{\tau}(s) \tilde{\xi}(s)) \tilde{\alpha}$ at $\tilde{x}(s) \in \tilde{\mathcal{P}}(s)$ such that, at the bifurcation point $\tilde{x}(0)=x(\bar{s}), \tilde{\xi}(0)=\xi(\bar{s}), \tilde{\alpha}^{2}=\alpha$ and $\tilde{\tau}(0)=\tau(\bar{s}) / 2$. Here, $\tilde{\alpha}=(312) \kappa_{2} R_{2}(\pi / 2)$ has order $\tilde{\ell}=12$. This corresponds to a bifurcation increasing the spatio-temporal symmetry $\mathbb{Z}_{6}\left(\alpha_{I I}\right)$ in 
the co-rotating frame to $\mathbb{Z}_{12}(\tilde{\alpha})$. The bifurcation, marked as "PF" in the third plot of Figure 1 , occurs at

$$
\begin{array}{ll}
q_{1}=(0.5545,-0.3628,0.2530), & q_{2}=(-0.4921,0.3094,0.3679), \\
p_{1}=(0.3616,0.1920,-0.7436), & p_{2}=(0.5237,0.3138,0.6587),
\end{array}
$$

the period in the frame rotating with velocity $\omega^{\text {rot }}=0.7305$ around the $e_{2}$-axis is $T=9.626$, the second component of the angular momentum is $\mu_{2}=1.576$, and $\mu_{1}=\mu_{3}=0$. The linearization $\mathrm{D} \tilde{\sigma}(0) \Phi_{x}^{\tilde{\tau}(0)}(\tilde{x}(0))$ at the bifurcation point has four eigenvalues 1 , two eigenvalues -1 and the eigenvalues

$$
\lambda_{1}=-2.1, \quad \lambda_{2}=-0.48, \quad \lambda_{3,4}=0.45 \pm \mathrm{i} 0.89, \quad \lambda_{5,6}=-0.55 \pm \mathrm{i} 0.83 .
$$

The first and second plot of Figure 1 show the continuation of the Figure Eight at zero momentum up to the bifurcation point "PF". For each computed rotating eight solution the motion of the first body in the $\left(q_{1,1}, q_{1,2}\right)$-plane and $\left(q_{1,1}, q_{1,3}\right)$-plane, respectively, is depicted in the respective co-rotating frame in which the solution becomes periodic. The Figure Eight trajectory of the first body lies in the $\left(q_{1,1}, q_{1,3}\right)$-plane, therefore it corresponds to a line in the second plot. We can see from the second plot that at the bifurcation point the solution is invariant under rotations by $\pi / 2$ about the $e^{2}$ axis.
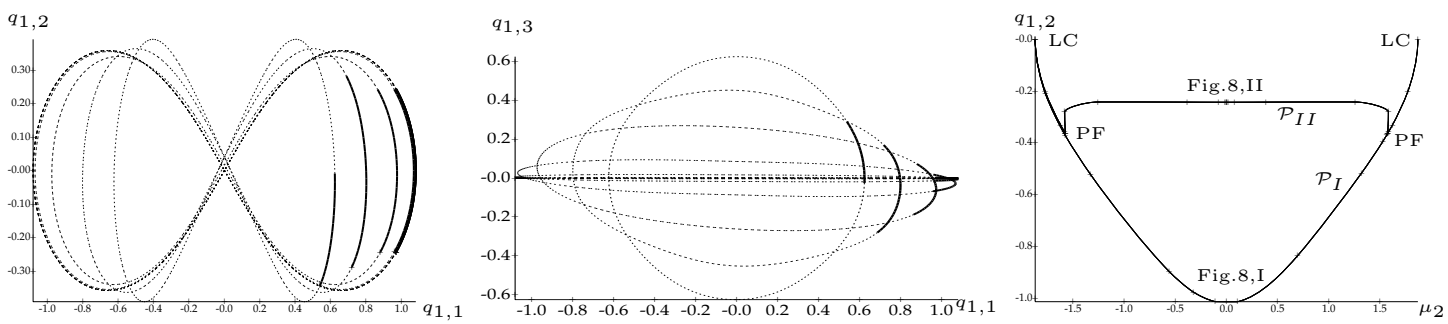

Figure 1: Symmetry-increasing bifurcation of the type II rotating eights.

The third plot of Figure 1 shows the bifurcation diagram as a graph of $q_{1,2}$ over $\mu_{2}$. The continuation starts on the type II branch $\mathcal{P}_{I I}(E, \nu)$ from the Figure Eight at $\mu_{2}=0$ (denoted "Fig.8 II" in the plot). As indicated by the label, the family bifurcating at "PF" lies on the type I family $\mathcal{P}_{I}(E, \nu)$ of rotating eights. This branch ends at a relative Lyapunov centre bifurcation in the Lagrange relative equilibrium at $\mu_{2}=1.869$, marked as "LC" in the plot. At the relative Lyapunov centre bifurcation the relative period $\bar{\tau}_{I}^{L C}$ of the type I family and the relative period $\tilde{\tau}^{L C}$ of the bifurcating family of RPOs are identical, but the periods $T_{I}^{L C}$ and $\tilde{T}^{L C}$ in their respective co-rotating frames satisfy $\tilde{T}^{L C}=2 \bar{T}_{I}^{L C}$. The rotation frequencies and the driftsymmetries of both rotating choreographies in their co-rotating frames are also different. If we continue the bifurcating family back-wards to momentum $\mu_{2}=0$ then we obtain the original Figure Eight solution (denoted "Fig.8 I" in the second plot of Figure 1), but with non-vanishing rotation frequency and period $2 \bar{T}$ in the co-rotating frame. Here, $\bar{T}$ is the period of the Figure Eight solution.

To understand this note that relative periods of RPOs are independent of the coordinate frame, but the period in the co-rotating frame and the drift-symmetry in the co-rotating frame differ for different co-rotating frames (Remark 2.15).

The type I family $\mathcal{P}_{I}(s), s=(E, \nu)$, of rotating eights at $x_{I}(s) \in \mathcal{P}_{I}(s)$ is transformed into the family $\tilde{\mathcal{P}}(s)$ of rotating choreographies bifurcating from the type II rotating eights at $\tilde{x}(s)$ 
by applying the following operations: First, the symmetry $\gamma:=(213) R_{1}(\theta) \alpha_{I}^{j}$ is applied onto $x_{I}(s)$, where $\alpha_{I}=\kappa_{1}(231)$ is the drift symmetry in the co-rotating frame of the RPO $\mathcal{P}_{I}(s)$ at $x_{I}(s)$. Moreover, $\theta \in \mathbb{R}$ and $j \in\{1, \ldots, 5\}$ are arbitrary. These operations correspond to a shift on the $\Gamma_{\bar{\mu}}$-orbit of $x_{I}(s)$ and a time shift, respectively, which are not determined by our algorithm, see (2.29). By this operation, the drift symmetry in the co-rotating frame becomes $\gamma \alpha_{I} \gamma^{-1}=\kappa_{1}(312)$. Then we rotate $\gamma x_{I}(s)$ by $R_{3}(\pi / 2)$, which transforms the drift symmetry in the co-rotating frame to $\kappa_{2}(312)$. Finally, we change the rotation frequency $\omega_{I}^{\text {rot }}(s)$ of the RPO $\mathcal{P}_{I}(s)$ to the rotation frequency $\tilde{\omega}^{\mathrm{rot}}(s)$ such that $\left(\tilde{\omega}^{\mathrm{rot}}(s)-\omega_{I}^{\mathrm{rot}}(s)\right) \tau_{I}(s)=\pi / 2$. This transforms the drift symmetry of the type I rotating eight $\mathcal{P}_{I}(s)$ in the co-rotating frame into the drift symmetry $\tilde{\alpha}=R_{2}(\pi / 2) \kappa_{2}(312)$ in the co-rotating frame of the RPOs bifurcating from the type II rotating eights, and the period $T_{I}(s)=\ell_{I} \tau_{I}(s)=6 \tau_{I}(s)$ in the co-rotating frame to $\tilde{T}(s)=\tilde{\ell} \tau_{I}(s)=12 \tau_{I}(s)=2 T_{I}(s)$ and explains the above observations.

If we continue the branch of type I rotating choreographies from the original Figure Eight in the momentum component $\mu_{1}$ fixing the energy, this connection between the type I and type II family of rotating eights corresponds to a relative period-doubling and a symmetry-breaking pitchfork bifurcation in the co-rotating frame at $\mu_{1}=1.576$. In this case the bifurcating branch $\tilde{\mathcal{P}}_{I I}(s)$ has drift symmetry $\tilde{\alpha}_{I I}=\alpha_{I}^{2}=(312)$ of order $\tilde{\ell}_{I I}=3$ in the co-rotating frame. If we continue the bifurcating branch $\tilde{\mathcal{P}}_{I I}(s)$ back to the Figure Eight solution at $\mu_{1}=0$, then the Figure Eight on this branch has non-vanishing rotation frequency and period $\bar{T} / 2=\tilde{\ell}_{I I} \bar{\tau}$ in this co-rotating frame. Denote the rotation frequency of $\tilde{\mathcal{P}}_{I I}(s)$ by $\tilde{\omega}_{I I}^{\text {rot }}(s)$. Applying the above transformation on the branch of type I rotating choreographies amounts to conjugating $\tilde{\alpha}_{I I}$ with $R_{3}(\pi / 2)(213)$ and changing the rotation frequency $\tilde{\omega}_{I I}^{\text {rot }}(s)$ of the RPO $\tilde{\mathcal{P}}_{I I}(s)$ to $\omega_{I I}^{\text {rot }}(s)$ where $\left(\omega_{I I}^{\text {rot }}(s)-\tilde{\omega}_{I I}^{\text {rot }}(s)\right) \tau_{I I}(s)=\pi$. In this way we retrieve the drift symmetry $\alpha_{I I}$ of the type II rotating eight in the original co-rotating frame, as expected.

\subsection{Turning points and relative period doubling bifurcations of type III rotating choreographies}

As reported in [24], the type III family of planar rotating choreographies can be continued to negative momentum values $\mu_{3}<0$ at fixed energy $\bar{E}=-0.1287$ and, after coming close to a collision, this family undergoes a relative period doubling bifurcation at momentum $\mu_{3}=-6.638$. Note that, in order to continue the family near the collision in [24] we increased the size of the RPOs by $c=10$ thus decreasing the energy by a factor of 0.1 using the scaling symmetry (4.3) of the three body problem.

A more detailed investigation revealed that along the primary branch further relative perioddoubling bifurcations (at momentum $\mu_{3}=-6.414$ and $\mu_{3}=-6.557$ ) and a turning point (at $\mu_{3}=-6.663$ ) occur before the relative period doubling bifurcation point at $\mu_{3}=-6.638$, see Figure 2 .

At the turning point (marked "TP" in the first panel of Figure 2) through

$$
q_{1}=(1.508,-0.173), \quad q_{2}=(-9.314,5.953), \quad p_{1}=\left(0.017,0.100, \quad p_{2}=(0.162,0.236),\right.
$$

the RPO has period $T=299.0$ in the co-rotating frame rotating with frequency $\omega^{\text {rot }}=0.0029$. In addition to the multipliers $\lambda_{1,2,3,4,5,6}=1$ its linearization in the co-rotating frame $\mathrm{D}_{x} \sigma \Phi_{\tau}(x)$ has eigenvalues $\lambda_{7}=-3.7, \lambda_{8}=-0.27$.

The type III rotating choreography, as continued from the Figure Eight, is on the lower part of the (unlabelled) primary solution branch in the first plot of Figure 2. The second plot of Figure 2 shows this solution at momentum $\mu_{3}=-6.2$, and the third plot shows it after the turning point, on the upper branch of the bifurcation diagram, at momentum $\mu_{3}=-6.2$. Note that the linearization at the RPO in the second plot has, in addition to four eigenvalues 

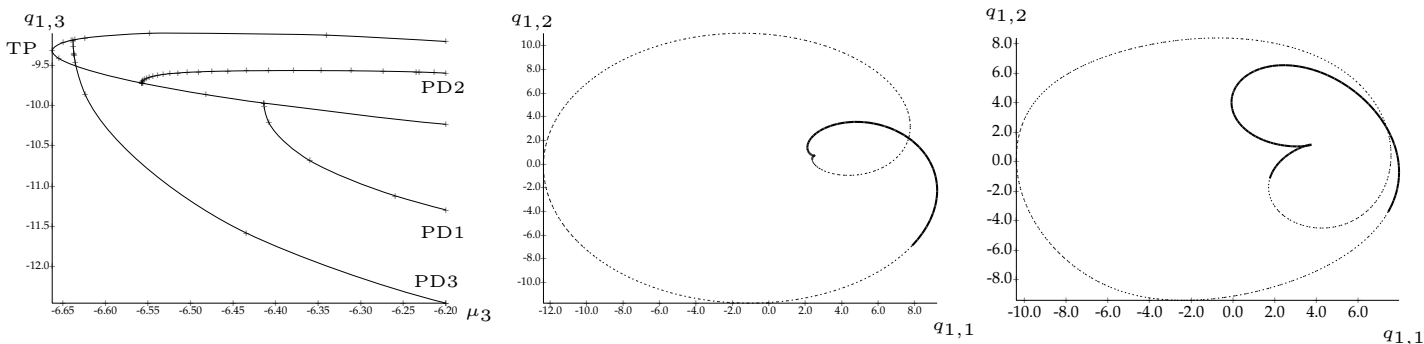

Figure 2: Turning points and relative period doubling bifurcation of the type III rotating choreographies; the second picture shows the primary solution on the lower branch, the third picture the primary solution on the upper branch, both at $\mu_{3}=-6.2$.

$\lambda_{1,2,3,4}=1$, one pair on the unit circle and two real negative eigenvalues. The linearization at the RPO in the third plot has, in addition to four eigenvalues $\lambda_{1,2,3,4}=1$, two real positive eigenvalues and one pair on the unit circle. When the turning point is approached from the lower part of the primary branch then one pair of eigenvalues of the linearization switches from a pair on the unit circle to a pair on the real line in a collision at $\lambda_{5,6}=1$, cf. Proposition 2.34.
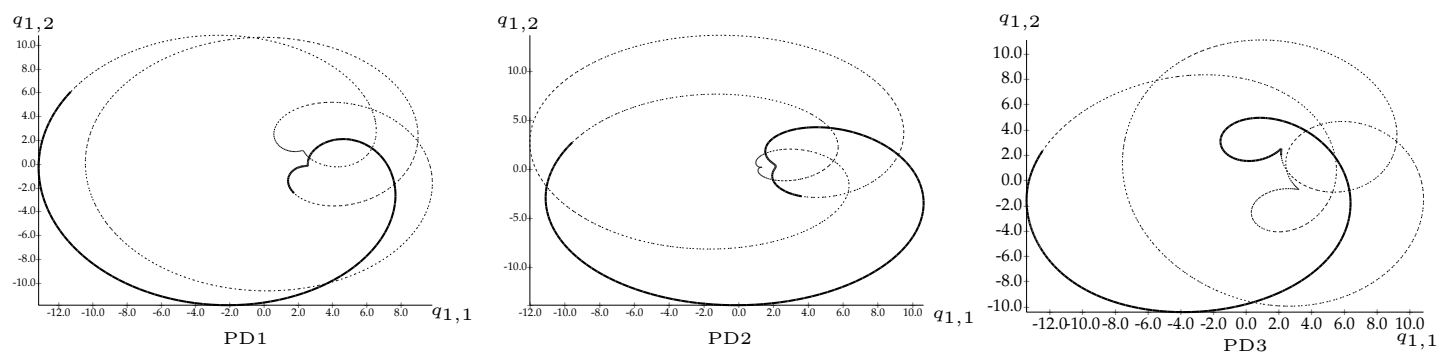

Figure 3: RPOs bifurcating from the type III family of rotating choreographies at momentum $\mu_{3}=-6.2$.

The first relative period doubling bifurcation (the emanating branch is marked "PD1" in the first plot of Figure 2) is at momentum $\mu_{3}=-6.414$, and the RPO passes through

$$
q_{1}=(2.021,0.169), \quad q_{2}=(-9.965,6.353), \quad p_{1}=(-0.009,0.107), \quad p_{2}=(0.162,0.209)
$$

and has period $T=225.5$ in a frame rotating with frequency $\omega^{\text {rot }}=0.0166$. In addition to four eigenvalues $\lambda_{1,2,3,4}=1$ and two eigenvalues $\lambda_{5,6}=-1$ its linearization has the eigenvalues $\lambda_{7}=-7.4, \lambda_{8}=-0.14$, and the pair of eigenvalues -1 passes from the unit circle to the real axis as $\mu_{3}$ is decreased. Pane PD1 of Figure 3 shows the solution on branch PD1 at momentum $\mu_{3}=-6.2$. In addition to four eigenvalues $\lambda_{1,2,3,4}=1$, the linearization at this solution has four real positive eigenvalues.

The second relative period doubling bifurcation (the emerging branch is marked "PD2" in the first plot of Figure 2) is at momentum $\mu_{3}=-6.557$, and the RPO passes through

$$
q_{1}=(1.762,0.022), \quad q_{2}=(-9.718,6.199), \quad p_{1}=(-0.003,0.101), \quad p_{2}=(0.163,0.222)
$$

and has period $T=246.4$ in a frame rotating with frequency $\omega^{\text {rot }}=0.0119$. In addition to four eigenvalues $\lambda_{1,2,3,4}=1$ and two eigenvalues $\lambda_{5,6}=-1$ its linearization has the eigenvalues 
$\lambda_{7}=-8.3, \lambda_{8}=-0.12$, and the pair of eigenvalues -1 passes from the real axis to the unit circle as $\mu_{3}$ is decreased. Pane PD2 of Figure 3 shows the solution on branch PD2 at momentum $\mu_{3}=-6.2$. In addition to four eigenvalues $\lambda_{1,2,3,4}=1$, the linearization at this solution has one positive real pair of eigenvalues and one pair on the unit circle.

The third relative period doubling bifurcation point (the emanating branch is marked "PD3" in the first plot of Figure 2) at momentum $\mu_{3}=-6.638$, which we already reported in [24], passes through

$$
q_{1}=(1.482,-0.348), \quad q_{2}=(-9.178,5.833), \quad p_{1}=(0.029,0.112), \quad p_{2}=(0.159,0.237)
$$

and has period $T=325.9$ in a frame rotating with frequency $\omega^{\text {rot }}=-0.00049$. In addition to four eigenvalues $\lambda_{1,2,3,4}=1$ and two eigenvalues $\lambda_{5,6}=-1$ its linearization has the eigenvalues $\lambda_{7}=20.2$ and $\lambda_{8}=0.05$. Pane PD3 of Figure 3 shows the solution on branch PD3 at momentum $\mu_{3}=-6.2$. In addition to four eigenvalues $\lambda_{1,2,3,4}=1$, the linearization at this solution has four negative eigenvalues.

\section{Conclusion}

In this paper we presented methods for detecting and computing certain critical points, including symmetry breaking and symmetry increasing bifurcations, of Hamiltonian relative periodic orbits with regular drift-momentum pair in the case of compact symmetry groups. The bifurcations we analyzed occur generically during the path-following of RPOs under the assumption that isotropy groups are trivial. We applied our results to rotating choreographies in the three-body problem.

Note that a systematic theory for all generic bifurcations of Hamiltonian RPOs does not yet exist. Consequently, a systematic numerical treatment of all such bifurcations is yet to be developed. For dissipative differential equations generic bifurcations of symmetric periodic orbits have been classified by Lamb and Melbourne [10] and a general bifurcation theory for RPOs of dissipative systems has been developed in [21].

A next step would be to develop numerical methods for the computation of generic symmetry changing bifurcations of RPOs of dissipative systems, which break spatial as well as spatiotemporal symmetries, and then to extend these methods to bifurcations of Hamiltonian RPOs which break discrete spatial symmetries. Note that bifurcations of Hamiltonian RPOs breaking continuous isotropy are much more difficult to analyze as the momentum map $\mathbf{J}$ is in general not surjective near such points. Preliminary results on bifurcations of Hamiltonian relative equilibria breaking continuous isotropy have been obtained for example in $[8,15]$, see also references therein.

In the bifurcations that we analyze in this paper we assume that the RPO at the bifurcation has a generic drift-momentum pair. The numerical treatment of bifurcations from RPOs with singular drift-momentum pair is as yet an open problem.

\section{Acknowledgements}

The authors thank Mark Roberts for stimulating discussions and comments. CW thanks the Freie Universität Berlin for their hospitality during the preparation of the manuscript, and acknowledges funding by the Leverhulme Foundation. FS and CW were supported by EPSRC grant EP/D063906/1. 


\section{References}

[1] R. Abraham and J.E. Marsden. Foundations of Mechanics. Second edition. Revised, enlarged, reset. The Benjamin/Cummings Publishing Company, Massachusetts, 1978.

[2] T. Bröcker and T. tom Dieck. Representations of Compact Lie Groups. Graduate Texts in Mathematics, vol. 98, Springer, New York, Berlin, Heidelberg, Tokyo, 1985.

[3] A. Chenciner, J. Féjoz, and R. Montgomery. Rotating Eights I: the three $\Gamma_{i}$ families. Nonlinearity 18, 1407-1424, 2005.

[4] A. Chenciner, R. Montgomery. A remarkable periodic solution of the three body problem in the case of equal masses. Annals of Mathematics 152, 881-901, 2000.

[5] B. Fiedler. Global Bifurcation of Periodic Solutions with Symmetry. Lecture Notes in Mathematics. Springer Verlag, Berlin, Heidelberg, New York, 1988.

[6] J. Galán, F.J. Muñoz-Almaraz, E. Freire, E. Doedel and A. Vanderbauwhede. Stability and bifurcation of the figure- 8 solution of the three-body system. Phys. Rev. Lett. 88(24), 241101-241105, 2002.

[7] M. Golubitsky, I. Stewart, D. Schaeffer. Singularities and Groups in Bifurcation Theory, volume 2. Springer-Verlag, Berlin, Heidelberg, New York, 1988.

[8] A. Hernandez and J.E. Marsden. Regularization of the amended potential and the bifurcation of relative equilibria. J. of Nonlinear Sci. 15, 93-132, 2005.

[9] Y. Kuznetsov. Elements of Applied Bifurcation Theory. Applied Mathematical Sciences, vol. 112. Springer, New York, 1995.

[10] J. Lamb and I. Melbourne. Bifurcation from discrete rotating waves. Arch. Rat. Mech. Anal. 149, 229-270, 1999.

[11] J.E. Marsden and T.S. Ratiu. Introduction to Mechanics and Symmetry. Springer-Verlag, New York, Berlin, Heidelberg, 1994.

[12] K.R. Meyer and G.R. Hall. Introduction to Hamiltonian dynamical systems and the N-body problem. Springer-Verlag, New York, 1992.

[13] J. Montaldi. Persistance d'orbites périodiques relatives dans les systèmes Hamiltoniens symétriques. C.R. Acad. Sci. Paris, Série I 324, 353-358, 1997.

[14] F.J. Muñoz-Almaraz, E. Freire, J. Galán, E. Doedel and A. Vanderbauwhede. Continuation of periodic orbits in conservative and Hamiltonian systems. Physica D 181(1,2), 1-38, 2003.

[15] J.-P. Ortega. Relative normal modes for nonlinear Hamiltonian systems. Proc. Royal Soc. Edinb., Sect. A, 133(3), 675-704, 2003.

[16] G. Patrick and R.M. Roberts. The transversal relative equilibria of Hamiltonian systems with symmetry. Nonlinearity 13, 2089-2105, 2000.

[17] R.M. Roberts, C. Wulff, and J. Lamb. Hamiltonian systems near relative equilibria. J. Differential Equations 179, 562-604, 2002.

[18] F. Schilder, C. Wulff, A. Schebesch. SYMPERCON - a package for the numerical continuation of symmetric periodic orbits. user manual, University of Surrey, 2008. 
[19] C. Wulff. Persistence of relative equilibria in Hamiltonian systems with non-compact symmetry. Nonlinearity 16, 67-91, 2003.

[20] C. Wulff. Persistence of Hamiltonian relative periodic orbits. J. Geom. Phys. 48, 309-338, 2003.

[21] C. Wulff, J.S.W. Lamb, and I. Melbourne. Bifurcations from relative periodic solutions. Erg. Th. Dyn. Syst. 21, 605-635, 2001.

[22] C. Wulff and M. Roberts. Hamiltonian systems near relative periodic orbits. SIAM J. Appl. Dyn. Syst. 1, 1-43, 2002.

[23] C. Wulff and A. Schebesch. Numerical continuation of symmetric periodic orbits. SIAM J. Appl. Dyn. Syst. 435-475, 2006.

[24] C. Wulff and A. Schebesch. Numerical continuation of Hamiltonian relative periodic orbits. J. Nonlinear Science 18(4), 343-390, 2008. 